JUNTA DE CIENCIAS NATURALES DE BARCELONA

\title{
INDEX SEMINUM
}

QUAE

HORTUS BOTANICUS MUSEI BARCINONENSIS SCIENT. NAT. MUTUA COMMUTATIONE OFFERT

1927

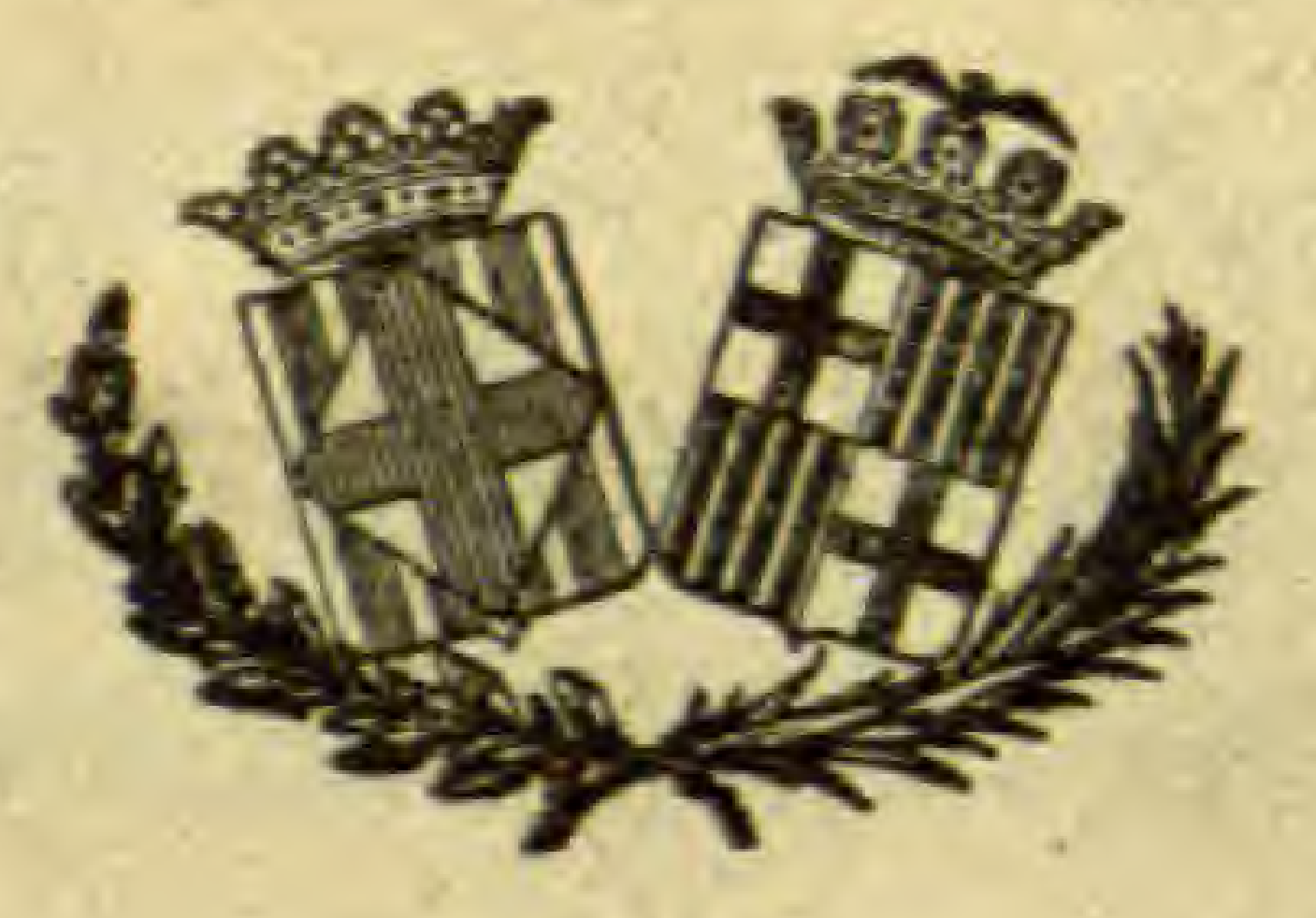

BARCINONIS

EX TYPIS ELZEVIRIANA DICTIS

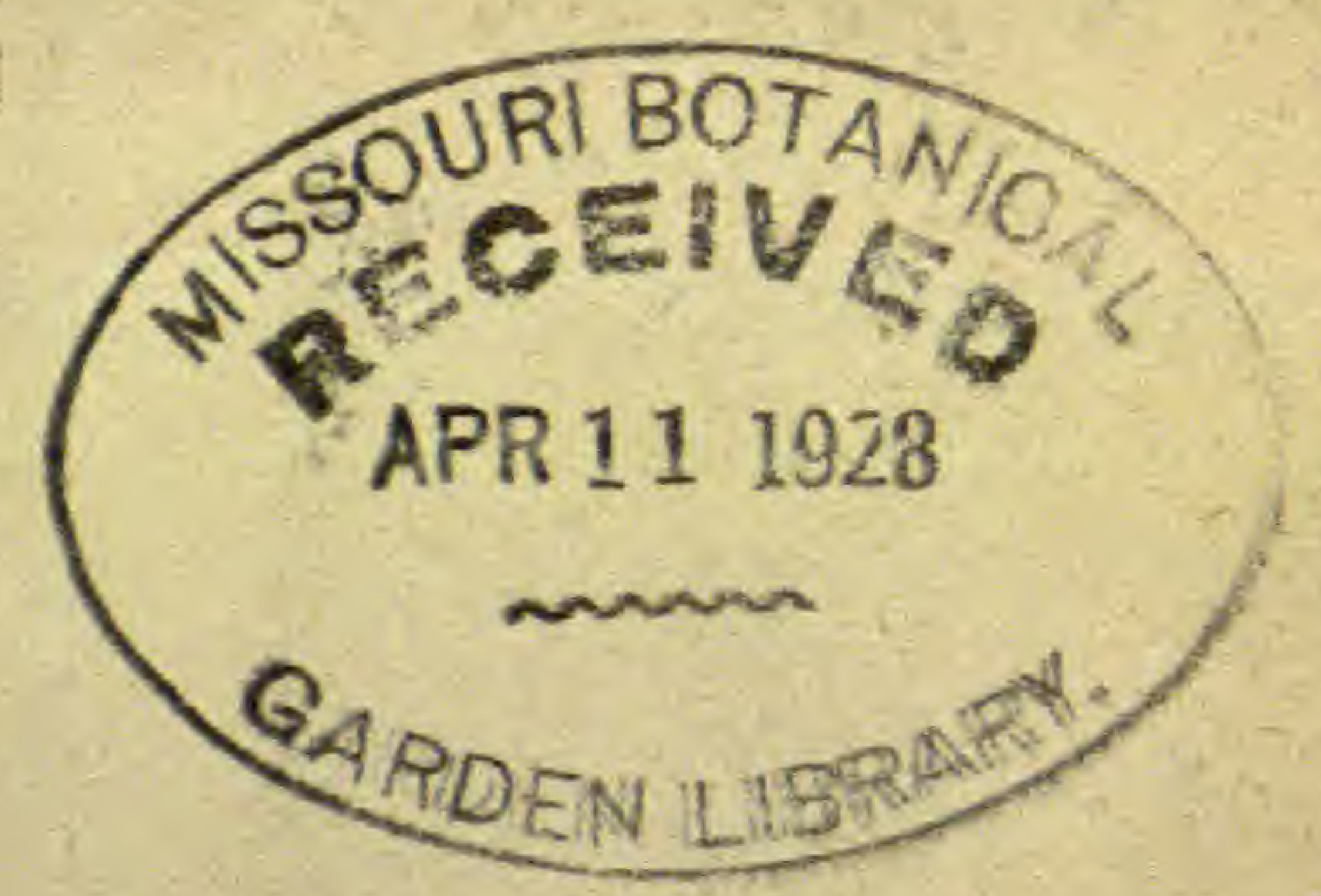



IN nostro hoc humili Viridario 1 Botanico Musei Barcinonensis, nuper erecto, alias plantas colere nequimus nisi quae nostra studia propias accedant.

Quare loco magnae seminum varietatis, haec nostra nunc praebemus, unicuique applicando locum originis qui in nostro archivo fideliter invenietur inscriptus. 



\section{INDEX SEMINUM}

QUAE HORTUS BOTANICUS MUSEI BARCINONENSIS SCIENT. NAT. MUTUA COMMUTATIONE OFFERT.

\section{Gramineae}

Dactylis glomerata L. var. maritima Hack. - Ebuso, in parva insula Esspardell de 1'Esspartar dicta.

\section{Araceae}

Biarum carratracense (Haens.) F. Q. - Carratraca (Regno Granat.), 1. clas.

Biarum tenuifolium (L.) Schott. - Benaocaz (Baetica).

\section{Liliaceae}

Merendera montana (L.) Lge. - Sierra Tejeda (Regno Granat.) Allium moschatum L. - San Guim (Catalaunia).

Allium nigrum L. - Atlante medio.

Allium pyrenaicum Costa et Vay. - Talaixà (Pyrenaeis), 1. clas. Allium stramineum Boiss. et Reut. var. xericiense P. Lara. Tarifa (Baetica).

Urginea undulata (Desf.) Stein. - Alcira (Regno Valent.)

Scilla obtusifolia Poir. - Salou (Catalaunia).

Ornithogalum Reverchonii Lge. - Grazalema (Baetica).

Ornithogalum tenuifolium Guss. - Ebuso.

EDITUS D. I MART. I928 


\section{Amaryllidaceae}

Narcissus dubius Gouan - Plana de S. Jordi (Catalaunia).

Narcissus poeticus L. - Montseny (Catalaunia).

Narcissus serotinus L. - Jerez de la Frontera (Baetica).

\section{Caryophyllaceae}

Arenaria balearica L. - Puig Major (Insula Majore).

Silene glauca Pourr. - Hifac (Regno Valent.)

Silene hifacensis Rouy - Ebuso.

Silene psammitis Link - Inter Monda et Ojén (Regno Granat.) Lychnis coeli-rosa (L.) Desv. - Inter Estepona et Casares (Regno Granat.)

Tunica Saxifraga (L.) Scop. var. grandiflora Goir. - E1 Chorro, c. Málaga (Regno Granat.)

Dianthus Seguieri Chaix - Girona (Catalaunia).

Saponaria officinalis L. - Barcelona.

\section{Ranunculaceae}

Delphinium Staphysagria L. - Frumentaria insula.

Clematis campaniflora Brot. - Despeñaperros (Marianis montibus).

Ranunculus blepharicarpos Boiss. - Esparteros, c. Morón (Baetica).

Ranunculus sceleratus L. - Miranda de Ebro (Castella vet.)

- $\quad$ Ceratocephalus falcatus (L.) Pers. - Cervera (Catalaunia).

Thalictrum aquilegifolium L. - Montseny (Catalaunia).

\section{Papaveraceae}

Papaver rupifragum Boiss. et Reut. - Grazalema (Baetica)' 1. clas. 


\section{Cruciferae}

Aethionema ovalifolium Boiss. - Dornajo (Sierra Nevada),

1. clas.

Thlaspi Prolongoi Boiss. - Grazalema (Baetica).

Alyssum minimum L. - Aranjuez (Castella) 1. clas.

Alyssum spinosum L. - Sierra Nevada (Hispania).

Isatis Djurdjurae Coss. - Yebel Lerz (Atlante rhiphaeo).

Carrichtera annua (L.) Prant1 - Frumentaria insula.

Vella pseudocytisus L. - Aranjuez (Castella), 1. clas.

Vella spinosa Boiss. - Dornajo (Sierra Nevada), 1. clas.

Eruca sativa Lamk. - Cervera (Catalaunia).

Sinapis alba L. - Albanchez (Regno Giennense).

Sinapis longirrostris Boiss. - Despeñaperros (Castella), 1. clas. Diplotaxis catholica (L.) D. C. var. bipinnatifida Kze. - Cádiz (Baetica).

Diplotaxis siifolia Kze. - S. Roque (Baetica), 1. clas.

Coincya rupester Rouy - Alcaraz (Regno Murcico), 1. clas.

Moricandia Ramburei Webb - Algar (Baetica).

Arabis leptopoda Pau et Font Quer. - Badú (in Atlante rhiphaeo).

\section{Capparidaceae}

Capparis spinosa L. var. inermis Savi - Horta, pr. Barcelona.

\section{Droseraceae}

Drosophyllum lusitanicum (L.) Link - Chiclana (Baetica).

\section{Rosaceae}

Sanguisorba hybrida (L.); Poterium hybridum I., sp. p1., ed. I, pág. 994; P. agrimonioides Spach. - Los Términos, c. Grazalema (Baetica). 


\section{Leguminosae}

Lupinus angustifolius L. - Inter Nerja et Torre del Mar (Regno Granat.)

Genista biflora (Desf.) DC.; Spartium biflorum Desf. - Ebuso. Genista catalaunica (Webb) Rouy; Sarothamnus catalaunicus Webb - Montalegre, c. Barcelona, 1. clas.

Genista dorycniifolia F. Q. - Ebuso.

Genista Grosii F. Q.; G. dorycniifolia var. Grosii F. Q. - Ebuso. Genista linifolia L. - Alcalá de los Gazules (Baetica).

Ononis arborescens Desf. var. remotiflora Pau et F. Q. - In montibus supra Targuist (Atlante rhiphaeo).

Ononis reclinata L. - Montgó (Regno valentino).

Ononis saxicola Boiss. et Reut. - Cerro de S. Cristóbal, c. Grazalema (Baetica). 1. clas.

Ononis speciosa Lag. - Sierra de Cómpeta (Regno Granat.)

Ononis subcordata Cav.; O. crotalarioides Coss. - Sierra Mágina (Regno Giennense).

Medicago arborea L. var, citrina F, Q. - In parva insula Espartar dicta (Ebuso).

Astragalus baeticus L. - S. Fernando (Baetica). Astragalus scorpioides Pourr. - Madrid, 1. clas.

Biserrula Pelecinus I. - Málaga (Regno Granat).

Scorpiurus vermiculatus. L. - Chiclana (Baetica).

Ornithopus compressus L. - Canillas de Albaida (Regno Granat.)

Ornithopus durus Cav. - Veger (Baetica).

Hippocrepis balearica Jacq. - Insula Majore.

Hippocrepis Salzmannii Boiss. Reut. - Sanlúcar de Barrameda (Baetica).

Onobrychis eriophora Desv. - Alcalá de los Gazules (Baetica). Vicia Godronii Rouy - Els Tres Salts, c. Manresa (Catalaunia). Lathyrus latifolius I. - Ubrique (Baetica).

Anthyllis hamosa Desf. - San Fernando (Baetica). Anthyllis podocephala Boiss. - Sierra de Algodonales (Baetica). 


\section{Geraniaceae}

Erodium Sanguis-Christi Sennen - Ametlla, Plana de S. Jordi (Catalaunia), 1. clas.

\section{Zygophyllaceae}

Peganum Harmala L. - Chiprana (Aragonia).

Zygophyllum Fabago L. - Benahadux (Regno Granat.)

\section{Cneoraceae}

Cneorum tricoccum L. - Ebuso.

\section{Euphorbiaceae}

Euphorbia glebulosa Coss. - Yebel Malmusi (Littore rhiphaeo). Euphorbia helioscopia L. forma bracteis capsulisque nigro-purpureis. - Balaguer (Catalaunia).

Euphorbia Lagascae Spreng. - Calp (Regno Valent.)

Euphorbia rupicola Boiss. - Sierra Blanquilla, c. Cortes (Baetica).

\section{Malvaceae}

Lavatera rotundata Laz. et Tub. - Aranjuez (Castella), 1. clas. Lavatera trimestris L. - Ubrique (Baetica).

\section{Guttiferae}

Hypericum balearicum L. - Insula Majore. 


\section{Cistaceae}

Cistus Bourgeauanus Coss. - Chiclana (Baetica), 1. clas.

Cistus populifolius L. var. lasiocalyx Willk. - Jimena de la Frontera (Baetica).

Helianthemum aegyptiacum Mill. - Aranjuez (Castella).

\section{Violaceae}

Viola Demetria Prol. - Cerro de S. Cristóbal c. Grazalema (Baetica).

\section{Onagraceae}

Onothera stricta Led. - La Línea (Baetica).

\section{Umbelliferae}

Bifora testiculata (L.) Spreng. - Ebuso.

Smyrnium perfoliatum L. - Güejar (Sierra Nevada).

Amarintea trifida (Mill.) Samp.; Cachrys laevigata Lam. - Sierra

Nevada (Hispania).

Heterothaenia thalictrifolia Boiss. - Benaocaz (Baetica).

Crithmum maritimum L. - Sitges (Catalaunia).

Ferula communis L. - Güejar (Sierra Nevada).

Peucedanum Cervaria L. - Girona (Catalaunia).

Pastinaca lucida Gouan - Aumallutx (Insula Majore).

Pastinaca silvestris Mill. - Tornabous (Catalaunia).

Thapsia garganica L. - E.buso.

Thapsia villosa L. - Banyeres (Regno Valentino).

\section{Primalaceae}

Lysimachia Ephemerum L. - Anglès (Catalaunia). Lysimachia minoriscensis Rodr. - Insula Minore. 


\section{Plumbaginaceae}

Statice insignis Coss. - Benahadux (Regno Granat.)

\section{Asclepiadaceae}

Gomphocarpus fruticosus (L.) R. Br. - Barcelona.

Caralluma europaea (Guss.) N. E. Br. var. confusa F. Q. - Cabo de Gata (Regno Granat.)

Caralluma Hesperidum Maire - Axdir (In littore rhiphaeo).

\section{Borraginaceae}

Omphalodes brassicifolia (Lag.) Boiss. - Sierra Blanquilla, c. Cortes (Baetica).

Anchusa calcarea Boiss. - Veger (Baetica).

Anchusa sempervirens L. - Hoyos del Espino, in Sierra de Gredos (Hispania).

Cerinthe major L., a typica (Cerinthe flore rubro purpurascente, Bauh., Pin. 258, ex Linné). - S. Fernando (Baetica).

\section{Labiatae}

Ajuga Iva L. - Ebuso.

Teucrium bracteatum Desf. - Targuist (Atlante rhiphaeo).

Sideritis glauca Cav. - Oriola (Regno valentino), 1. clas. atque unico.

Sideritis Lacaitae F. Q. - Despeñaperros (Castella), 1. unico.

Sideritis leucantha Cav. - Crevillent (Regno Valentino).

Nepeta Apuleji Ucr. - Paterna (Baetica).

Cleonia lusitanica L. - Ubrique (Baetica).

Stachys recta L. - Riera d'Osor (Catalaunia).

Salvia candelabrum Boiss. - Convento de las Nieves (Regno Granat.), 1. clas. 
Salvia ceratophylloides Ard. - Inter Terreti et Stravorino (Calabria).

Salvia Barrelieri Et1.; S. inamoena Vah1. - Ubrique (Baetica).

\section{Solanaceae}

Atropa Belladona L. - Serra de 1'Almuçara (Catalaunia). Triguera Osbeckii (L.) Willk. - Medina Sidonia (Baetica). Hyoscyamus albus L. - Torredenbarra (Catalaunia). Physalis Alkekengi L. - San Guim (Catalaunia). Solanum sodomaeum L. - S. Fernando (Baetica). Datura Metel L. - Calafell (Catalaunia). Datura Stramonium L. - S. Pere de Vilamajor (Catalaunia).

\section{Scrophulariaceae}

Celsia Barnadesii (Vahl) G. Don. var. mauritanica Pau - Yebel Malmusi (In littore riphaeo). Celsia simuata Cav. - S. Fernando (Baetica).

Linaria Cavanillesii Chav. - Montgó, c. Dènia (Regno Valentino).

Linaria Clementei Haens. - Sierra de Mijas (Regno Granat.) Linaria delphinoides Gay - Sierra de Majarreina (Extremadura).

Linaria gobantesiana Coincy - Gobantes (Regno Granat.), 1. clas.

Linaria hirta (L.) Moench - Nerja (Regno Granat.) Linaria Pelliceriana (L.) Mill. - Cap de Creus (Calataunia).

Linaria platycalyx Boiss. - Peñón de Ronda (Baetica), 1. clas. Linaria tingitana Boiss. Reut. - Tetauen (Yebala). Antirrhinum Charidemii Lge. - Cabo de Gata (Regno Granat.), 1. clas. et unico.

Antirrhinum molle L. - Montsec (Pyr. Catalauniae). Antirrhinum valentinum F. Q. - Xeresa (Regno Valent.), 1. clas. 
Chaenorrhinum crassifolium (Cav.) Lge. - Bocairent (Regno Valent.)

Scrophularia sambucifolia L. - Alcalá de los Gazules (Baetica). Scrophularia Scorodonia L. - Trevélez (Sierra Nevada).

Digitalis purpurea L. var. mauritanica Humb. et Maire - Yebel Iguermalez (In Atlante rhiphaeo).

\section{Plantaginaceae}

Plantago Loeflingii L. - Miranda de Ebro (Castella vet.)

\section{Campanulaceae}

Legousia conferta (Moench) Samp. - Porto (Lusitania).

\section{Compositae}

Phagnalon Carolipaui F. Q., sp. nov.: Suffruticosum, divaricato ramosum, foliis linearibus ut in Phagn. sordido, involucris glabris, squamis adpressis, valde inaequalibus, obtusis, pallide fuscescentibus, inferioribus brevibus, oblongis et late scariosis apice rotundato et obtuso, intimis linearibus; flosculis roseis, achaenis adpresse pilosis, parvis. Affinis Ph. calycino (Cav.) sed gracilior, ramis tenuis, divaricatis, foliis angustioribus, integris, capitulis minoribus, squamarum partibus non scariosis truncatis vel obtusis, achaenis majoribus setisque pappi magis incrassatis, etc. - Badú (Atlante rhiphaeo).

Anthemis maritima L. - Cádiz (Baetica).

Anthemis nobilis L. - Grazalema (Baetica).

Glossopappus chrysanthemoides Kze. - Utrera (Baetica).

Senecio leucanthemifolius Poir. - Tetauen (Yebala).

Senecio petraeus Boiss. et Reut. - Grazalema (Baetica), 1. clas. Arctotis calendulacea Willd. - Cádiz (Baetica).

Cirsium leptophyllum (P. et F. Q.) F. Q.; Chamaepence leptophylla Pau et Font Quer - Ad pedem montis Iguermalez (Atlante rhiphaeo), 1. clas. 
Cynara humilis L. - Ubrique (Baetica).

Mariana eburnea (Coss. et Dur.) Villar, in "Ibérica», I925, pág. 33r. - Aranjuez (Castella).

Amberboa muricata (L.) DC. - Torre del Mar, c. Málaga (Regno Granat.)

Centaurea algeriensis Coss. et Dur. - S. Juan de las Minas, c. Melilla.

Centaurea Clementei Boiss. - Ronda (Regno Granat.)

Centaurea diluta Ait. - Paterna (Baetica).

Centaurea leucantha Pourr. var. grandifolia F. Q. - Frumentaria insula, 1. clas.

Centaurea pullata L. - Tetauen (Yebala).

Centaurea salmantica L. var. valdemorensis (Cut.) - Verdú (Catalaunia).

Carthamus dianius (Webb) Coincy - Ebuso.

Catananche caerulea L. - San Guim (Catalaunia).

Geropogon glaber L. - Tarifa (Baetica).

Scorzonera pygmaea Sibt. et S. - Tizzi Iffri (Atlante rhiphaeo).

Taraxacum tomentosum Lge. - San Guim (Catalaunia).

Crepis tingitana (Salzm.) Ball; Crepis baetica Lge. - Picacho de Alcalá de los Gazules (Baetica).

Barcinone, 20 novembris 1927.

DR. P. FONT QUER

Botanicae Sect. Scient. Nat. Barc. Musei Curator

J. SANTAMaria

Horti Magister
E. GRos

Botanicus Collector, horti assistens

Adresse: P. Font QuER. - Apartado 593. - BARCELONA 




\section{MHK \\ A.H.Jr. \\ Q $33+6$}

JUNTA DE CIENCIAS NATURALES DE BARCELONA

2

\section{INDEX SEMINUM}

QUAE

HORTUS BOTANICUS MUSEI BARCINONENSIS SCIENT. NAT. MUTUA COMMUTATIONE OFFERT

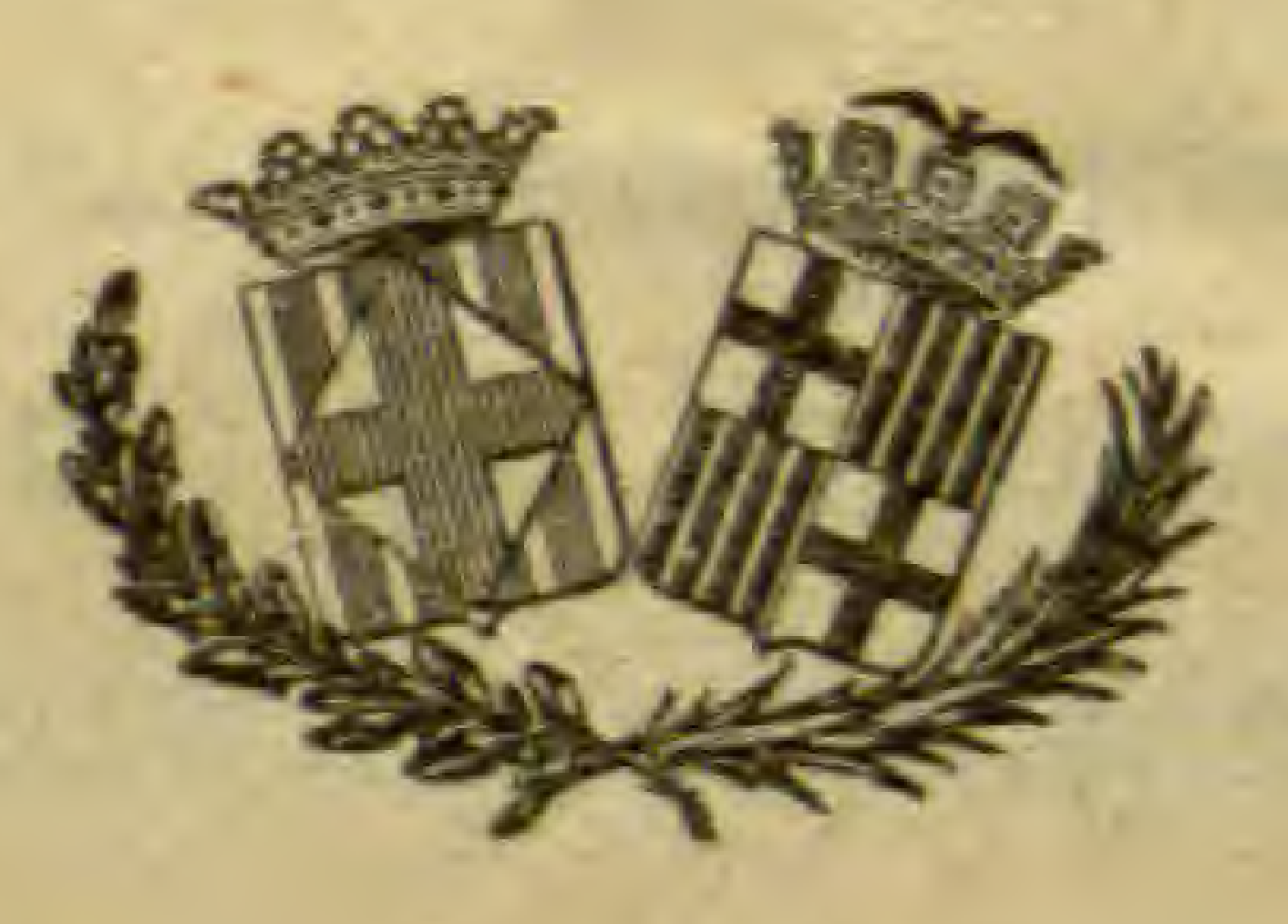

BARCINONIS

EX TYPIS ELZEVIRIANA DICTIS

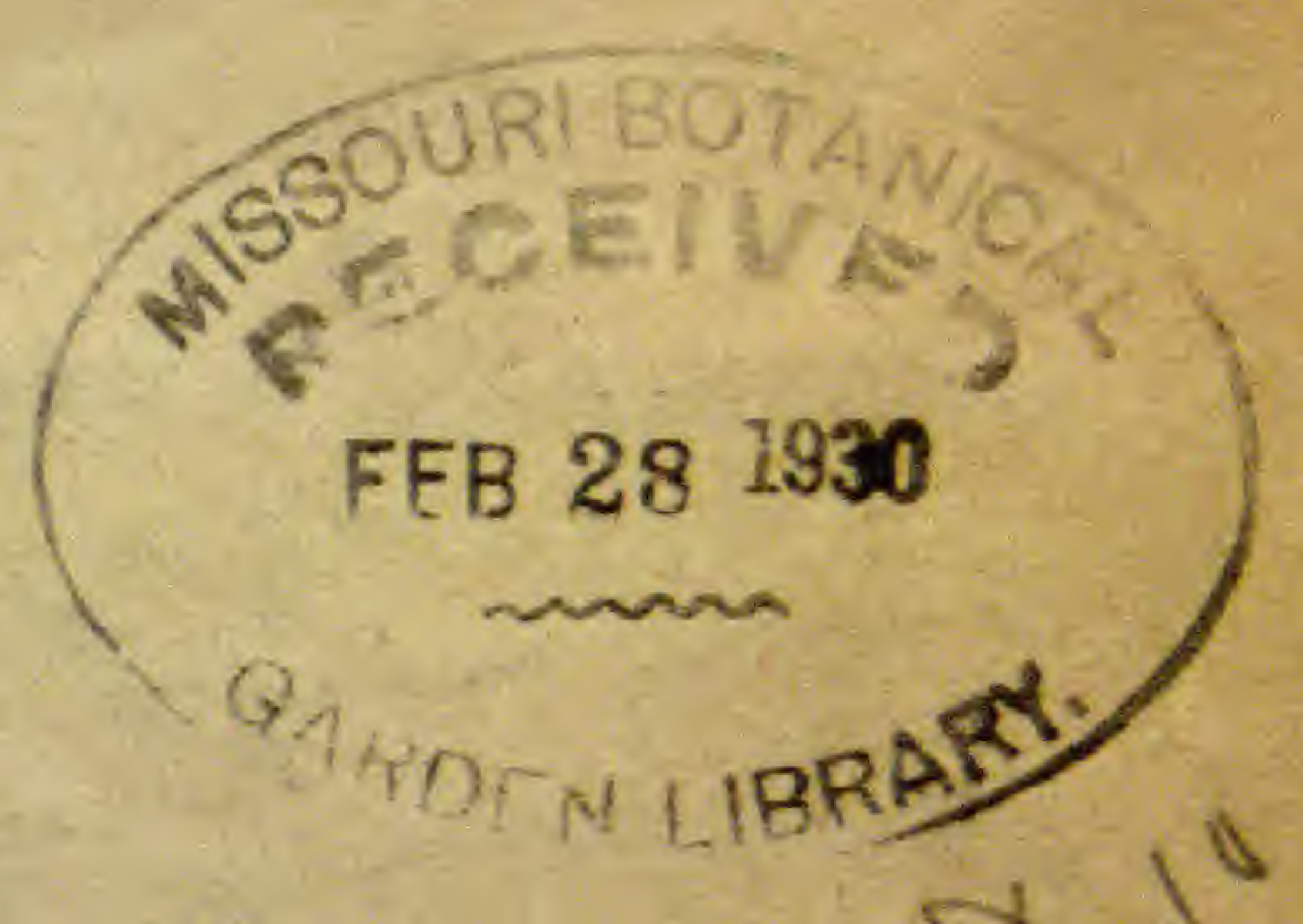



$T^{N}$ nostro hoc humili Viridario

1 Botanico Musei Barcinonensis nuper erecto, alias plantas colere nequimus nisi quae nostra studia propias accedant.

Quare loco magnae seminum varietatis, haec nostra nunc praebemus, unicuique applicando locum originis qui in nostro archivo fideliter invenietur inscriptus. 



\section{INDEX SEMINUM}

QUAE HORTUS BOTANICUS MUSEI BARCINONENSIS SCIENT. NAT. MUTUA COMMUTATIONE OFFERT.

\section{Juncaginaceae}

Triglochin laxiflora Guss. - Chiclana (Baetica).

\section{Gramineae}

Dactylis glomerata L. var. maritima Hack. - Ebuso, in parva insula Espardell de 1'Espartar dicta.

Oplismenus undulatifolius R. S. - Olot (Catalaunia).

\section{Araceae}

Biarum carratracense (Haens.) F. Q. - Carratraca (Regno Granat.), 1. clas.

Biarum tenuifolium (L.) Schott. - Benaocaz (Baetica).

\section{Liliaceae}

Merendera montana (L.) Lge. - Sierra Tejeda (Regno Granat.) Colchicum lusitanicum Brot. - Benaocaz.

Allium moschatum L. - San Guim (Catalaunia).

Allium nigrum $\mathrm{L}$. - Atlante medio.

Allium pyrenaicum Costa et Vay.- Talaixà (Pyrenaeis), 1. clas. Allium stramineum Boiss. et Reut. var. xericiense P. Lara. -

Tarifa (Baetica).

× Lilium Martagon L. - Montserrat.

Urginea undulata (Desf.) Stein. - Alcira (Regno Valent.)

\ Scilla obtusifolia Poir. - Salou (Catalaunia).

EDITUS D. JAN. I930 
6

Junta de Ciencias Naturales de Barcelona

Hyacinthus amethystimus I, var. Fontqueri (Pau) F. Q.; H. Fontqueri Pau. - Ports de Tortosa (Catalatnia), 1. clas.

Ornithogalum Reverchonii Lge. - Grazalema (Baetica).

Ornithogalum tenuifolium Guss. - Ebuso.

\section{Amaryllidaceae}

Leucojum autumnale L. - Despeñaperros (Castella Nova).

Narcissus dubius Gouan. - Plana de S. Jordi (Catalaunia).

Narcissus poeticus L. - Montseny (Catalaunia).

Narcissus serotinus L. - Jerez de la Frontera (Baetica).

Narcissus juncifolius Lag. - Motserrat.

Tapeinanthus humilis (Cav.) Herb. - Jerez.

\section{Iridaceae}

Iris Xiphium L. - Ia Barraca d'Aigües vives (Regno Valentino).

Gynandriris Sisyrinchium Parl. - Pto. de Sta. Maria (Baetica).

\section{Caryophyllaceae}

Cerastium dichotomum I. - Targuist (Imp. Maroc).

Arenaria balearica L. - Puig Major (Insula Majore).

Arenaria Pomelü Munby - Tizi Ifri, c. Targuist (Atlante rhiphaeo).

Arenaria spathulata Desf. - Yebel Malmusi (Bokoya - Littore rhiphaeo).

Silene disticha Willd. - Xauen (Gomara - Imp. maroccano).

Silene glauca Pourr. - Hifac (Regno Valent.)

Silene hifacensis Rouy - Ebuso.

Silene Martyi Emb. et Maire - Zarkat (Atlante rhiphaeo).

Silene Otites L. - Eistany d'Ibars (Catalaunia).

Silene psammitis Link - Inter Monda et Ojén (Regno Granat.) 
Lychnis coeli-rosa (L.) Desv. - Inter Estepona et Casares (Regno Granat.)

Tunica Saxitraga (L.) Scop. var. granditlora Goir. - E1 Chorro, c. Málaga (Regno Granat.)

Dianthus Seguieri Chaix - Girona (Catalaunia).

Dianthus catalaunicus Pourr. - Montseny.

Saponaria officinalis I. - Barcelona.

\section{Ranunculaceae}

Isopyrum thalictroides L. - Olot.

Delphinium Staphysagria L. - Frumentaria insula.

Clematis campaniflora Brot. -- Despeñaperros (Marianis montibus).

Ranunculus blepharicarpos Boiss. - Essparteros, c. Morón (Baetica).

Ranunculus sceleratus L. - Miranda del Fibro (Castella vet.)

Thalictrum aquilegifolium $\mathrm{I}_{\text {. }}$ - Montseny (Catalaunia).

\section{Papaveraceae}

Papaver rupifragum Boiss. et Reut. - Grazalema (Baetica), 1. clas.

\section{Cruciferae}

Thlaspi Prolongoi Boiss. -. Grazalema (Baetica).

Alyssum granatense-Boiss. Reut. - Bu Meziat, c. Targuist (Atlante rhiphaeo).

Alyssum minimum L. - Aranjuez (Castella), 1. clas. Alyssum spinosum I. - Sierra Nevada (Hispania).

Psychine stylosa Desf. var, auriculata F. Q. - Axdir (Littore rhiphaeo). .

Isatis Djurdjurae Coss. - Yebel Lerz (Atlante rhiphaeo).

Carrichtera annua (L.) Prantl - Frumentaria insula, 
Vella pseudocytisus L. - Aranjuez (Castella), 1. clas. Vella spinosa Bois. - Dornajo (Sierra Nevada), 1. clas. Eruca sativa Lamk. - Cervera (Catalaunia).

Sinapis alba L. - Albanchez (Regno Giennense).

Sinapis longirrostris Boiss. - Despeñaperros (Castella), 1. clas. Diplotaxis catholica (L.) D. C. var. bipinnatifida Kze. - Cádiz (Baetica).

Diplotaxis siifolia Kze. - S. Roque (Baetica), 1. clas.

Coincya rupester Rouy - Alcaraz (Regno Murcico), 1. clas.

Moricandia Ramburei Webb - Algar (Baetica).

Arabis alpina I. - Peña Mayor de Mena (Cantabria).

Arabis auriculata Lamk, - Tizi Ifri, c. Tazguist (Atlante rhiphaeo).

Arabis leptopoda Pau el Font Quer. - Badú (in Atlante rhiphaeo).

Arabis parvula Duf. - Targuist (Atlante rhiphaeo).

\section{Capparidaceae}

Capparis spinosa I. var. inermis Savi - Horta, pr. Barcelona.

\section{Saxifragaceae}

Saxifraga Vayredana Luiz. - Montseny.

\section{Rosaceae}

Sanguisorba hybrida (L.) ; Poterium hybridum I., sp. pl., ed. I, pág. 994; P. agrimonioides Spach. - Los Términos, c. Grazalema (Baetica).

\section{Leguminosae}

Lupinus angustifolius I.-- Inter Nerja et Torre del Mar (Regno Granat.) 
Genista biflora (Desf.) DC.; Spartium biflorum Desf. - Ehuso. Genista catalaunica (Webb) Rouy; Sarothamnus catalaunicus Webb-Montalegre, c. Barcelona, 1. clas.

Genista dorycniifolia F. Q. - Ebuso.

Genista equisetiformis Spach - Málaga (Regno Granatense).

Genista Grosii F. Q.; G. dorycniitolia var. Grossii F. Q.-Ebuso. Genista linifolia L. - Alcalá de los Gazules (Baetica).

Ononis angustissima Lamk. - Mogador.

Ononis arborescens Desf. var. remotiflora Pau et F. Q. -- In montibus supra Targuist (Atlante rhiphaeo).

Ononis reclinata $\mathrm{L}_{\text {. }}$ - Montgó (Regno valentino).

Ononis saxicola Boiss. et Reut. - Cerro de S. Cristóbal, c. Grazalema (Baetica), 1. clas.

Ononis speciosa Lag. - Sierra de Cómpeta (Regno Granat.)

Ononis subcordata Cav.; $O$. crotalarioides Coss. - Sierra Mágina (Regno Giennense).

Trifolium atlanticum Ball - Zarkat (Atlante rhiphaeo).

Trifolium Tastetii F. Q. - Bu-Meziat, c. Targuist (Atlante rhiphaeo).

Medicago arborea I. var. citrina F. Q. - In parva insula Espartar dicta (E.buso).

Astragalus baeticus L. - S. Fernando (Baetica).

Astragalus scorpioides Pourr. -- Madrid, 1. clas.

Biserrula Pelecinus I. - Málaga (Regno Granat.)

Scorpiurus vermiculatus L. - Chiclana (Baetica).

Ornithopus compressus L. - Canillas de Albaida (Regno Granat.)

Ornithopus durus Cav. - Veger (Baetica).

Hippocrepis balearica Jacq. - Insula Majore.

Hippocrepis eriocarpa (Boiss.) Pau - Sierra tejeda (Regno Granatense), 1. clas.

Hippocrepis Salzmannii Boiss. Reut. - Sanlúcar de Barrameda (Baetica).

Onobrychis eriophora Desv. - Alcalá de los Gazules (Baetica). Hedysarum pallidum Desf. - S. Juan de las Minas, pr. Melilla. Vicia Godronii Rouy - Els Tres Salts, c. Manresa (Catalaunia). 
Lens culinaris Medik ssp. nigricans (M. B.) Thell.-Zarkat (Atlante rhiphaeo).

Anthyllis hamosa Desf. - San Fernando (Baetica). Anthyllis podocephala Boiss. - Sierra de Algodonales (Baetica).

\section{Geraniaceae}

Erodium Sanguis-Christi Sennen - Ametlla, Plana de S. Jordi (Catalaunia), 1. clas.

\section{Zygophyllaceae}

Peganum Harmala L. - Chiprana (Aragonia).

Zygophyllum Fabago L. - Benahadux (Regno Granat.)

\section{Cneoraceae}

Cneorum tricoccum L. - Ebuso.

\section{Euphorbiaceae}

Euphorbia glebulosa Coss. - Yebel Malmusi (Littore rhiphaeo). Euphorbia helioscopia L. forma bracteis capsulisque nigro-purpureis. - Balaguer (Catalaunia).

Euphorbia Lagascae Spreng. - Calp (Regno Valent.)

Euphorbia rupicola Boiss. - Sierra Blanquilla, c. Cortes (Baetica).

Mercurialis serratifolia (Ball) Pan - Badú (Atlante rhiphaeo).

\section{Malvaceae}

Lavatera rotundata Laz. et Tub. - Aranjuez (Castella), 1. clas. Lavatera trimestris L. - Úbrique (Baetica). 


\section{Guttiferae}

Hypericum balearicum L. - Insula Majore.

\section{Cistaceae}

Cistus Bourgeauanus Coss. - Chiclana (Baetica), 1. clas.

Cistus populifolius L. var. lasiocalyx Willk. - Jimena de la Frontera (Baetica).

Halimium atriplicifolium Spach - Ankod (Gomara - Imp. maroccano).

Helianthemum aegyptiacum Mill. - Aranjuez Castella).

\section{Violaceae}

Viola Demetria Prol. - Cerro de S. Cristóbal c. Grazalema (Baetica).

\section{Onagraceae}

Onothera stricta Led. - La Línea (Baetica).

\section{Umbelliferae}

Lagoecia cuminoides L. - Dos Hermanas (Baetica).

Bifora testiculata (L.) Spreng. - Ebuso.

Smyrnium perfoliatum I. - Güiejar (Sierra Nevada).

Amarintea trifida (Mill.) Samp.; Cachrys laevigata Lam.-Sierra

Nevada (Hispania).

Heterothaenia thalictrifolia Boiss. - Benaocaz (Baetica).

Crithmum maritimum L. - Sitges (Catalaunia).

Athamanta sicula L. - Bokoia (Littore rhiphaeo).

Ferula communis L. - Güejar (Sierra Nevada).

Peucedanum Cervaria L. - Girona (Catalaunia). 
Pastinaca lucida Gouan-- Aumallutx (Insula Majore).

Pastinaca silvestris Mill. - Tornabous (Catalaunia).

Thapsia garganica L. - Ebuso.

Thapsia villosa L. - Banyeres (Regno Valentino).

\section{Primulaceae}

Lysimachia Ephemerum L. --Anglés (Catalaunia).

Lysimachia minoriscensis Rodr. - Insula Minore.

\section{Plumbaginaceae}

Statice insignis Coss. - Benahadux (Regno Granat.)

\section{Asclepiadaceae}

Gomphocarpus fruticosus (I.) R. Br. - Barcelona.

Caralluma europae (Guss.) N. E. Br. var. contiusa F. Q. - Cabo de Gata (Regno Granat.)

Caralluma Hesperidum Maire - Axdir (In littore rhiphaeo).

\section{Borraginaceae}

Omphalodes brassicifolia (Lag.) Boiss. - Sierra Blanquilla, c. Cortes (Baetica).

Anchusa calcarea Boiss. - Veger (Baetica).

Anchusa sempervirens L. - Hoyos del Espino, in Sierra de Gredos (Hispania).

Cerinthe major $\mathrm{L}_{\text {.., }}$ a typica (Cerinthe flore rubro purpurascente,

Bauh., Pin. 258, ex Linné). - S. Fernando (Baetica).

\section{Labiatae}

Ajuga Iva I. - Ebuso.

Teucrium bracteatum Desf. - Targuist (Atlante rhiphaeo).

Teucrium lancifolium Boiss. - Galatzó (Insula Majore). 
Sideritis foetens Clem. - Almería, Barranco del Caballar. (Regno Granatense).

Sideritis glauca Cav. - Oriola (Regno valentino), 1. clas. atque. unico.

Sideritis Lacaitae F. Q. - Despeñaperros (Castella), 1. unico. Sideritis leucantha Cav. - Crevillent Regno Valentino).

Nepeta algeriensis De Noe - Alger.

Nepeta Apuleji U'cr. - Paterna (Baetica).

Cleonia lusitanica L. - Úbrique (Baetica).

Stachys recta $\mathrm{I}_{\text {. }}$ - - Riera d'Osor (Catalaunia).

Salvia aegyptiaca L. - Torres de Alcalá (Littore rhiphaeo).

Salvia candelabrum Boiss. - Convento de las Nieves (Regno Granat.), I. clas.

Salvia interrupta Schousb. - Xauen (Gomara. - Imp. Maroccanum).

Salvia Mouretii Batt. et Pitard. - Imp. Maroc. occid.

Salvia officinalis L. ssp. lavandulifolia Vahl. var. vellerea Cuatr. - Sierra Mágina Regno Giennense), 1. clas.

Xalvia ceratophylloides Ard. - Inter Terreti et Stravorino (Calabria).

Salvia Barrelieri Et1.; S. inamoena Vahl. - Ubrique (Baetica).

\section{Solanaceae}

Atropa Belladona L. - Serra de 1'Almuçara (Catalaunia).

Triguera Osbeckii (L.) Willk. - Medina Sidonia (Baetica).

Hyoscyamus albus L. - Torredembarra (Catalaunia).

Physalis Alkekengi L. - San Guim (Catalaunia).

Solanum sodomaeum L. - S. Fernando (Baetica).

Datura Metel L. - Calafell (Catalaunia).

Datura Stramonium L. - S. Pere de Vilamajor (Catalaunia).

\section{Scrophulariaceae}

Celsia Barnadesii (Vahl) G. Don. var. mauritanica Pau - Yebel Malmusi (In littore rhiphaeo). 
Celsia simuata Cav. - S. Fernando (Baetica).

Linaria arenaria DC. - Morbihan.

Linaria Cavanillesii Chav. - Montgó, c. Dènia (Regno Valentino).

Linaria Clementei Haens. - Sierra de Mijas (Regno Granat.)

Linaria delphinoides Gay - Sierra de Majarreina (Extremadura).

Linaria gobantesiana Coincy - Gobantes (Regno Granat.), 1. elas.

Linaria hirta ( $\left.\mathrm{I}_{\text {.. }}\right)$ Moench - Nerja (Regno Granat.)

Linaria Pelliceriana (I.) Mill. - Cap de Crens (Catalaunia).

Linaria platycalyx Boiss. - Peñón de Ronda (Baetica), 1. clas. Linaria rhiphatlantica F. Q. - Yebel Perz (Atlante rhiphaeo), 1. clas.

Linaria tingitana Boiss. Retit. - Tetatuen (Yebala).

Antirrhinum chrysothales F. Q. - Torres de Alcalá (Littore rhiphaeo).

Antirrhimum Charidemii Lge. - Cabo de Gata (Regno Granat.), 1. clas. et unico.

Antirrhinum molle L. - Montsec (Pyr. Catalauniae).

Antirrhinum valentinum F. Q. - Xeresa (Regno Valent.), 1. clas. Chaenorrhinum crassifolium (Cav.) I.ge. - Bocairent (Regno Valent.)

Scrophularia sambucifolia I. - Alcalá de los Gazules (Baetica).

Scrophularia Scorodonia L. - Trevélez (Sierra Nevada).

Scrophularia villosiuscula Emb. et Maire-Zarkat (Atlante rhiphaeo).

Digitalis purpurea L. var. mauritanica Humb. et Maire - Yebel Iguermalez (In Atlante rhiphaeo).

\section{Plantaginaceae}

Plantago Loeflingii L. - Miranda de Ebro (Castella vet.) 


\section{Dipsacaceae}

Scabiosa stellata L. - Tobarra (Albacete).

\section{Campanulaceae}

Legousia conferta (Moench) Samp. - Porto (Lusitania).

\section{Compositae}

Phagnalon Carolipaui F. Q. - Badú (Atlante rhiphaeo).

Phagnalon calycinum (Cav.) DC. - Mogador, 1. clas.

Anthemis maritima I. - Cádiz (Baetica).

Anthemis nobilis L. - Grazalema (Beatica).

Ormenis multicaulis Br. B1. et Maire. - Kenitra (Imp. Maroc.)

Glossopappus chrysanthemoides Kze. - Utrera (Baetica).

Leucanthemum paludosum (Poir.) Bonnet-Amekran, c. Axdir (Littore thiphaeo).

Senecio leucanthemifolius Poir. - Tetauen (Yebala).

Senecio petraeus Boiss. et Reut. - Grazalema (Baetica), 1. clas. Arctotis calendulacea Willd. - Cádiz (Baetica).

Cirsium lepthophyllum (P. et F. Q.) F. Q.; Chamaepence leptophylla Pau et Font Quer-Ad pedem montis Iguermalez (Atlante rhiphaeo), 1. clas.

Cynara humilis L. - Ubrique (Baetica).

Cynara algarbiensis Coss. subsp. hystrix (Ball.) F. Q.; C. Hystrix Ball - Inter Sok-et-Tnin et Targuist (Imp. Maroc.)

Mariana eburnea (Coss. et Dur.) Villar, in "Ibérica», I925. pág. 33r. - Aranjuez (Castella).

Amberboa muricata (L.) DC. - Torre del Mar, c. Málaga (Regno Granat.)

Centaurea algeriensis Coss. et Dur. - S. Juan de las Minas, c. Melilla. 
Centaurea Clementei Boiss. - Ronda (Regno Granat.)

Centaurea diluta Ait. - Paterna (Baetica).

Centaurea leucantha Pourr. var. grandifolia F. Q. - Frumentaria insula, 1. clas.

Centaurea pullata L. - Tetauen (Yebala).

Centaurea sálmantica L. var. valdemorensis (Cut.) - Verdú (Catalaunia).

Carthamus dianius (Webb) Coincy - Ebuso.

Kentrophyllum rhiphaeum F. Q. et Pau. - Axdir Littore rhiphaeo), 1. clas.

Catananche caerulea L. - San Guim (Catalaunia).

Geropogon glaber L. - Tarifa (Baetica).

Scorzonera pygmaea Sibt. et S. - Tizzi Iffri (Atlante rhiphaeo). Hypochaeris saldensis Batt. - Bougie, Cap. Carbon (Afr. bor.) Taraxacum tomentosum Lge. - San Guim (Catalaunia).

Crepis tingitana (Salzm.) Ball; Crepis baetica Lge. - Picacho de Alcalá de los Gazules (Baetica).

Barcinone, 3I decembris I929.

DR. P. FONT QUER

Botanicae Sect. Scient. Nat. Barc. Musci Curator

J. Santamaría Horti Magister
E. Gros

Botanicus Collector, horti assistens

Adresse: P. FONT QUER. - Apartado 593. - BARCELONA 
JUNTA DE CIĖNCIES NATURALS DE BARCELONA

\section{INDEX SEMINUM}

QUAE

HORTUS BOTANICUS MUSEI BARCINONENSIS

SCIENT. NAT. MUTUA COMMUTATIONE OFFERT
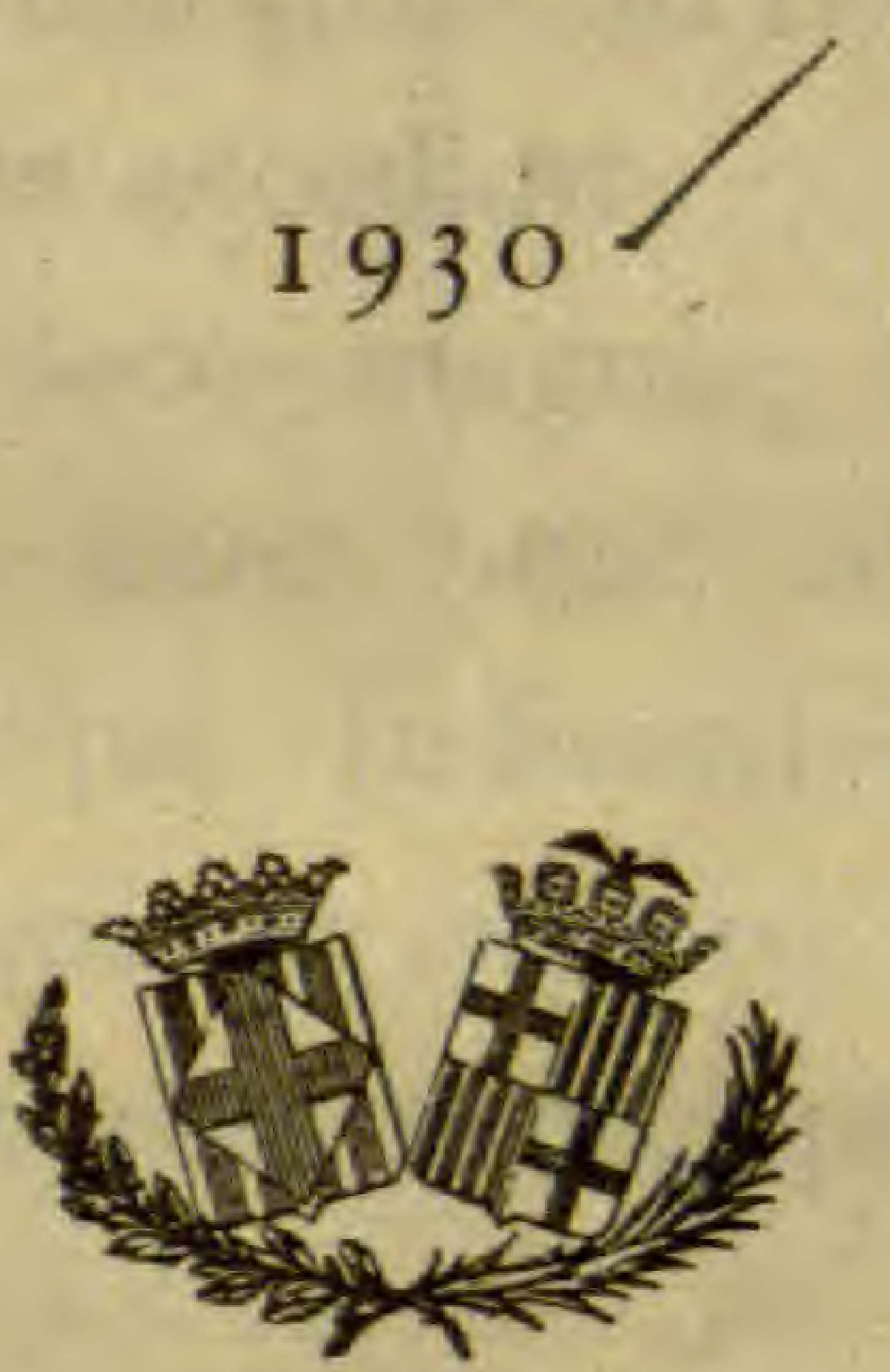


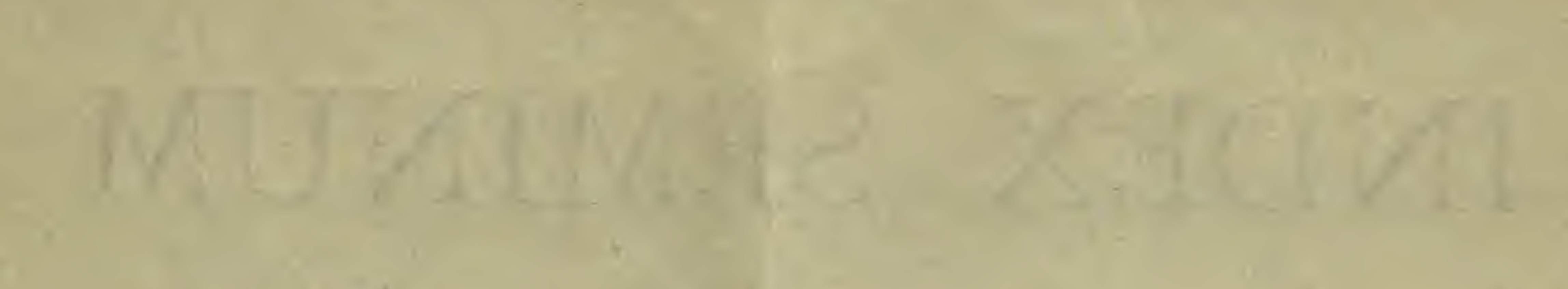


$I_{\text {Notanico Musei Barcinonensis }}^{\mathrm{N} \text { nostro hoc humili Viridario }}$ nuper erecto, alias plantas colere nequimus nisi quas ad nostra studia propius accedant.

Quare, loco magnae seminum varietatis, haec nunc offerimus, uniuscujusque indicantes locum originis, qui in nostro archivio fideliter invenitur inscriptus. 


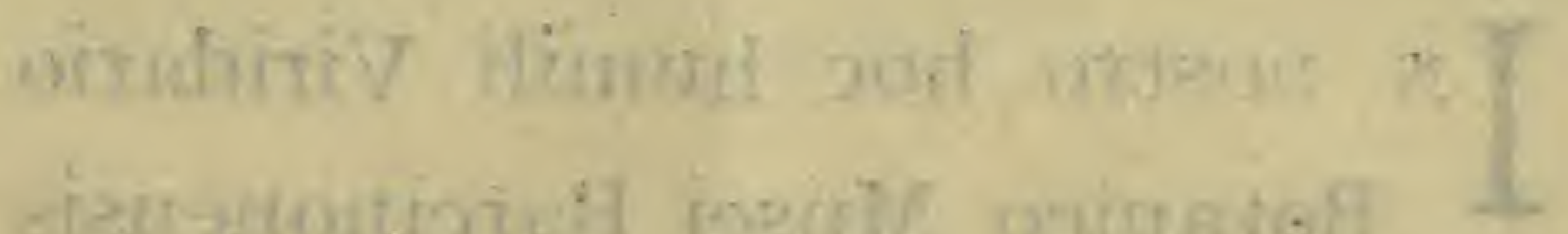

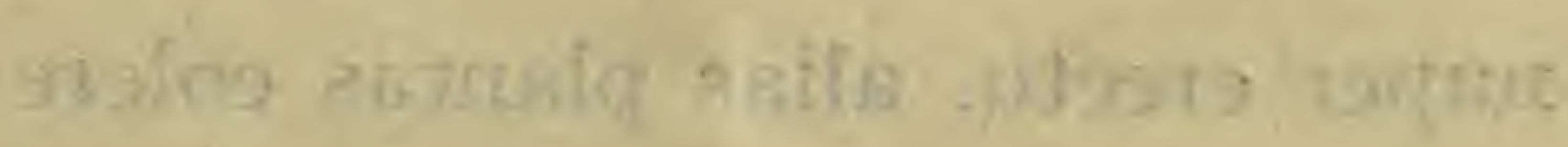

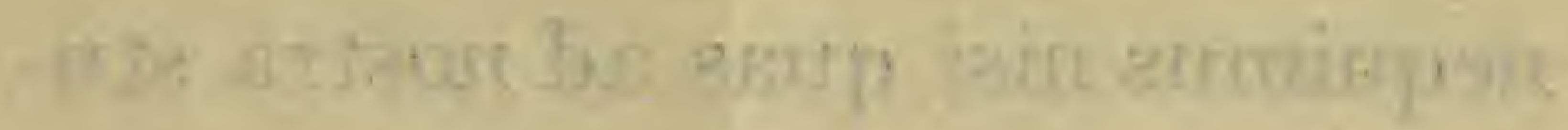

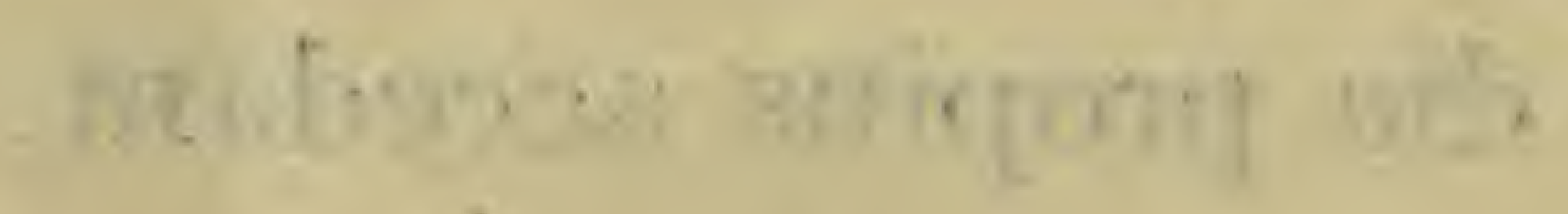

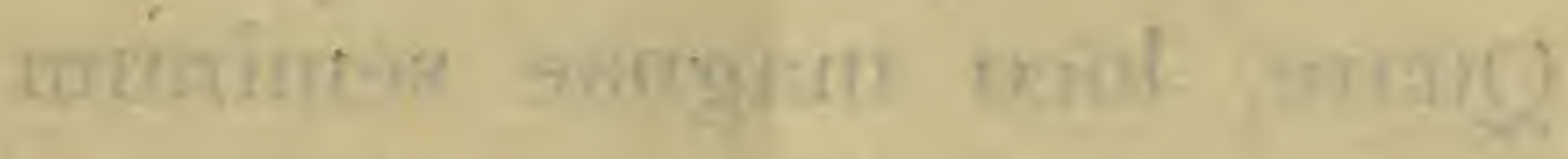

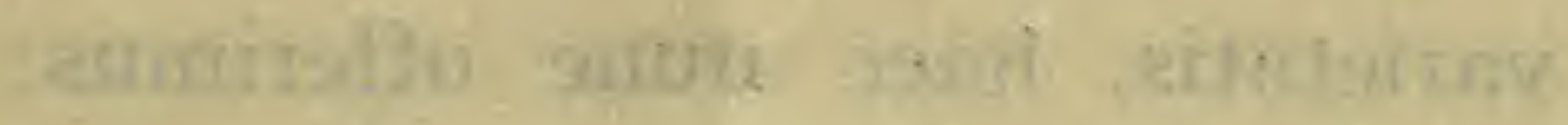

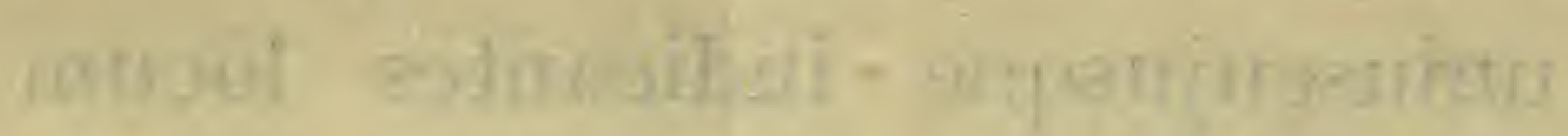

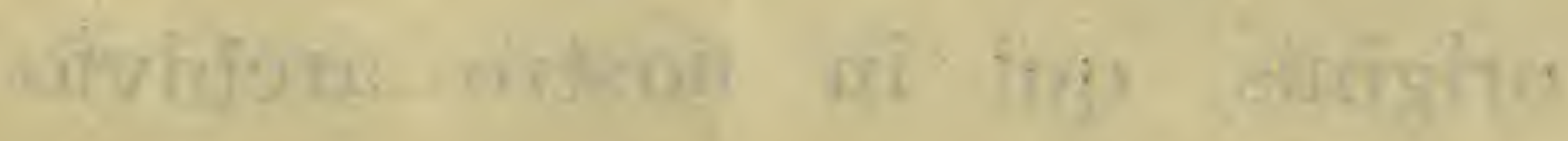

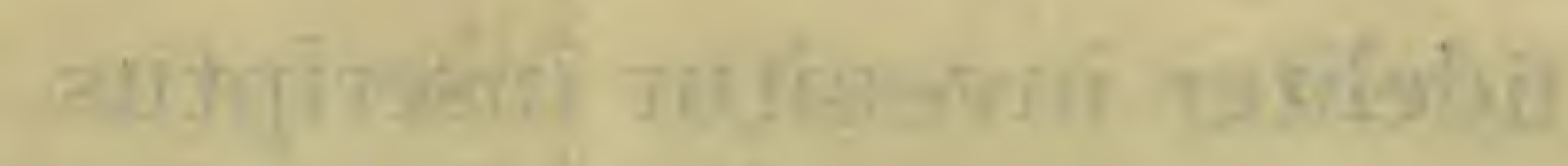




\section{INDEX SEMINUM}

QUAE HORTUS BOTANICUS MUSEI BARCINONENSIS SCIENT. NAT. MUTUA COMMUTATIONE OFFERT.

\section{Juncaginaceae}

Triglochin laxiflora Guss. - Chiclana (Baetica).

\section{Gramineae}

Dactylis glomerata L. var. maritima Hack. - Ebuso, in parva insula Espardell de l'Espartar dicta.

Festuca Font-Queri St.-Yv. - Tidiguin (Atlante rhiphaeo).

Oplismenus undulatifolius R. S. - Olot (Catalaunia).

\section{Araceae}

Biarum carratracense (Haens.) F. Q. - Carratraca (Regno Granat.), 1. clas.

Biarum tenuifolium (L.) Schott. - Benaocaz (Baetica).

\section{Liliaceae}

Merendera montana (L.) Lge. - Sierra Tejeda (Regno Granat.) Colchicum lusitanicum Brot. - Benaocaz.

Allium moschatum I. - San Guim (Catalaunia).

Allium nigrum I. - Atlante medio.

Allium pyrenaicum Costa et Vay. - Talaixà (Pyrenaeis), 1. clas. Allium stramineum Boiss. et Reut. var. xericiense P. Lara -

Tarifa (Baetica).

Lilium Martagon L. - Montserrat.

Urginea undulata (Desf.) Stein. - Alcira (Regno Valent.)

Scilla obtusifolia Poir. - Salou (Catalaunia). 
Scilla Paui Lac. - Sierra de Alcaraz.

Scilla Ramburei Boiss. - Chiclana (Baetica).

Hyacinthus amethystinus L. var. Font-Queri (Pau) F. Q.; H. Font-

Quevi Pav. - Ports de Tortosa (Catalaunia), 1. clas.

Ornithogalum Reverchonii L.ge. - Grazalema (Baetica).

Ornithogahum tenuifolium Guss. - Ebuso.

\section{Amaryllidaceae}

Leucojum autumnale I. - Despeñaperros (Castella Nova).

Narcissus dubius Gouan. - Plana de S. Jordi (Catalaunia).

Narcissus poeticus L. - Montseny (Catalaunia).

Narcissus serotinus L. - Jerez de la Frontera (Baetica).

Narcissus elegans Spach. var. fallax F. Q. - Bocoia (Litt. rhiphaeo).

Narcissus juncifolius Lag. - Montserrat.

Tapeinanthus humilis (Cav.) Herb. - Jerez.

\section{Iridaceae}

Iris alata Poir. - E1 Araix (Imp. Maroc.)

Iris Xiphium L. - La Barraca d'Aigües vives (Regno Valentino).

Iris tingitana Boiss. et Reut., vera. - R'gaia, c. Tandja, 1. clas. Gynandriris Sisyrinchium Parl. - Pto. de Sta. María (Baetica).

\section{Caryophyllaceae}

Minuartia maroccana Pau et F. Q. - Bocoia (Litt. rhiphaeo). Cerastium dichotomum I. - Targuist (Imp. Maroc).

Arenaria balearica L. - Puig Major (Insula Majore).

Arenaria Pomelii Munby - Tizi Ifri, c. Targuist (Atlante rhiphaeo).

Arenaria spathulata Desf. - Yebel Malmusi (Bocoia - Littore rhiphaeo).

Silene disticha Willd. - Xauen (Gomara - Imp. maroccano). 
Silene glauca Pourr. - Hifac (Regno Valent.)

Silene hifacensis Rouy - Ebuso.

Silene Martyi Emb. et Maire - Zarkat (Atlante rhiphaeo).

Silene Otites L. - Estany d'Ibars (Catalaunia).

Silene psammitis Link - Inter Monda et Ojén (Regno Granat.) Lychnis coeli-rosa (L.) Desv. - Inter Esstepona et Casares (Regno Granat.)

Tunica Saxifraga (L.) Scop. var. grandiflora Goir. - El Chorro,

c. Máiaga (Regno Granat.)

Dianthus Seguieri Chaix - Girona (Catalaunia).

Dianthus catalaunicus Pourr. - Montseny.

Saponaria officinalis L. - Barcelona.

\section{Ranunculaceae}

Isopyrum thalictroides L. - Olot.

Clematis campaniflora Brot. - Despeñaperros (Marianis montibus).

Ranunculus blepharicarpos Boiss. - Essparteros, c. Morón (Baetica).

Ranunculus sceleratus L. - Miranda de Ebro (Castella vet.)

Thalictrum aquilegifolium I. - Montseny (Catalaunia).

\section{Papaveraceae}

Papaver rupifragum Boiss. et Reut. - Grazalema (Baetica), 1. clas.

\section{Cruciferae}

Iberis gibraltarica I. - Tetauen (Imp. Maroc.)

Thlaspi Prolongoi Boiss. - Grazalema (Baetica).

Alyssum granatense. Boiss. Reut. - Bu Meziat, c. Targuist (Atlante rhiphaeo).

Alyssum spinosum I. Sierra Nevada (Hispania).

Psychine stylosa Desf. var. auriculata F. Q. - Axdir (Littore rhiphaeo). 
Carrichtera annua (L.) Prant1 - Frumentaria insula.

Succorvia balearica L. - Insula Majore (Bal.)

Vella pseudocytisus I. - Aranjuez (Castella), 1. clas.

Vella spinosa Bois. - Dornajo (Sierra Nevada), 1. clas.

Sinapis alba L. - Albanchez (Regno Giennense).

Sinapis longirrostris Boiss. - Despeñaperros (Castella), 1. clas.

Diplotaxis catholica (L.) D. C. var. bipinnatitida Kze. - Cádiz (Baetica).

Diplotaxis siifolia Kze. - S. Roque (Baetica), 1. clas.

Hemicrambe fruticulosa Webb - Tetaten (Imp. Maroc.), 1. clas. Coincya rupester Rouy - Alcaraz (Regno (Murcico), 1. clas. Draba hispanica L. - Dj. Lexhab (Imp. Maroc.)

Moricandia Ramburei Webb. - Algar (Baetica).

Avabis alpina L. - Peña Mayor de Mena (Cantabria).

Arabis auriculata Lamk. - Tizi Ifri, c. Targuist (Atlante rhiphaeo).

Arabis parvula Duf. - Targuist (Atlante rhiphaeo).

\section{Capparidaceae}

Capparis spinosa L. var. inermis Savi - Horta, pr. Barcelon a.

\section{Saxifragaceae}

Saxifaga Vayredana Luiz. - Montseny.

\section{Rosaceae}

Sanguisorba hybrida (L.) ; Poterium hybrudum L., sp. pl., ed. I, pág. 994; P. agrimonioides Spach. - Los Términos, c. Grazalema (Baetica).

\section{Leguminosae}

Genista biflora (Desf.) DC.; Spartium biflorum Desf. - Ebuso'. Genista catalaunica (Webb) Rouy; Sarothamnus catalunicus Webb-Montalegre. c. Barcelona, 1. clas. 
Genista dorycniifolia F. Q. - Ebuso.

Genista Grosii F. Q.; G. dorycniifolia var. Grosii F. Q. - Ebuso. Genista equisetiformis Spach. - Málaga (Regno Granatense).

Genista linifolia L. - Alcalá de los Gazules (Baetica).

Ononis angustissima Lamk. - Mogador.

Ononis reclinata L. - Montgó (Regno valentino).

Ononis speciosa Lag. - Sierra de Cómpeta (Regno Grant.)

Trifolitım atlanticum Ball. - Zarkat (Atlante rhiphaeo).

Trifolium Tastetii F. Q. - Bu-Meziat, c. Targuist (Atlante rhiphaeo).

Medicago arborea L. var. citrina F. Q. - In parva insula Espartar dicta (Ebuso).

Psoralea polystachya Poir. - E1 Araix (Imp. Maroc.)

Astragalus baeticus L. - S. Fernando (Baetica).

Astragalus hamosus L. var. ancocarpus (Pomel) - E1 Araix (Imp. Maroc.)

Astragalus lusitanicus Lam. -- E1 Araix (Imp. Maroc.)

Biserrula Pelecinus I. - Málaga (Regno Granat.)

Scorpiurus vermiculatus L. - Chiclana (Baetica).

Ornithopus compressus I, - Canillas de Albaida (Regno Granat.)

Hippocrepis balearica Jacq. - Insula Majore.

Hippocrepis eriocarpa (Boiss.) Pau. - Sierra Tejeda (Regno Granatense), 1. clas.

Hippocrepis Salzmannii Boiss, Reut. -- Sanlúcar de Barrameda (Baetica).

Onobrychis eriophora Desv. - Alcalá de los Gazules (Baetica). Hedysarum pallidum Desf. - S. Juan de las Minas, pr. Melilla. Hedysarum flexuosum L. - R'gaia, c. Tandja.

Vicia Godronii Rouy. - Eis Tres Salts, c. Manresa (Catalaunia). Anthyllis hamosa Desf. - San Fernando (Baetica).

Anthyllis podocephala Boiss. - Sierra de Algodonales (Baetica).

\section{Geraniaceae}

Erodium Sanguis-Christi Sennen - Ametlla, Plana de S. Jordi (Catalaunia), 1. clas. 


\section{Zygophyllaceae}

Peganum Harmala I. - Chiprana (Aragonia).

Zygophyllum Fabago I. - Benahadux (Regno Granat.)

\section{Cneoraceae}

Cneorum tricoccum $\mathrm{I}_{+}$. - Ebuso.

\section{Euphorbiaceae}

Euphorbia glebulosa Coss. - Yebel Malmusi (Littore rhiphaeo). Euphorbia helioscopia L. forma bracteis capsulisque nigro-purpureis. - Balaguer (Catalaunia).

Euphorbia rupicola Boiss. - Sierra Blanquilla, c. Cortes (Baetica).

Mercurialis serratifolia (Ball) Pau - Badú (Atlante rhiphaeo).

\section{Malvaceae}

Lavatera rotundata Laz. et Tub. - Aranjuez (Castella), 1. clas. Lavatera trimestris L. - Ubrique (Baetica).

\section{Guttiferae}

Hypericum balearicum L. - Instula Majore.

\section{Cistaceae}

Cistus Bourgeauamus Coss. - Chiclana (Baetica), 1. clas.

Cistus populifolius L. var. lasiocalyx Willk. - Jimena de la Frontera (Baetica).

Halimium atriplicifolium Spach. - Ankod (Gomara. - Imp. maroccano).

\section{Violaceae}

Viola Demetria Prol, - Cerro de S. Cristóbal c. Grazalema (Baetica). 


\section{Onagraceae}

Onothera stricta Led. - La Línea (Baetica).

\section{Umbelliferae}

Eryngium glaciale Bois. - Tidiguin (Atlante rhipaeo).

Tinguarra sicula (L.) Benth. et Hook. - Tetauen (Imp. Mar.)

Lagoecia cuminoides L: - Dos Hermanas (Baetica).

Bifora testiculata (L.) Spreng. - Ebuso.

Smyrnium perfoliatum I. - Güejar (Sierra Nevada).

Amarintea trifida (Mill.) Samp.; Cachrys laevigata L,am. - Sierra

Nevada (Hispania).

Magydaris panacifolia Lge. - El Araix (Imp. Maroc).

Heterothaenia thalictrifolia Boiss. - Benaocaz (Baetica).

Crithmum maritimum L. - Sitges (Catalaunia).

Angelica silvestris L. - Montseny (Catalaunia).

Capnophyllum peregrinum (L.) Lge. - R'gaia, c. Tandja.

Ferula communis I. - Güejar (Sierra Nevada).

Peucedanum Cervaria L. - Girona (Catalaunia).

Pastinaca lucida Gouan - Aumallutx-(Insula Majore).

Pastinaca silvestris Mill. - Tornabous (Catalaunia).

Thapsia garganica L. - Ebuso.

Thapsia villosa L. - Banyeres (Regno Valentino).

\section{Primulaceae}

Lysimachia minoriscensis Rodr. - Insula Minore.

\section{Plumbaginaceae}

Statice insignis Coss. - Benahadux (Regno Granat.)

\section{Asclepiadaceae}

Gomphocarpus fructicosus (L.) R. Br. - Barcelona.

Caralluma Hesperidum Maire - Axdir (In littore rhiphaeo). 
Junta de Ciencies Naturals de Barcelona

\section{Convolvulaceae}

Convolvulus Vidalii Pau - Xauen (Imp. Maroc.), 1. clas.

\section{Borraginaceae}

Omphalodes brassicifolia (Lag.) Boiss. - Sierra Blanquilla, c. Cortes (Baetica).

Anchusa calcarea Boiss. - Veger (Baetica).

Anchusa sempervirens L. - Hoyos del Fispino, in Sierra de Gredos (Hispania).

Cerinthe major L., $\alpha$ typica (Cerinthe flore rubro purpurascente,

Bauh., Pin. 258, ex Linné). - S. Fernando (Baetica).

Echium asturicum Lac. - Covadonga.

\section{Labiatae}

Ajuga Iva L. I E.buso.

Teucrium bracteatum Desf. - Targuist (Atlante rhiphaeo).

Teucrium lancifolium Boiss. - Galatzó (Insula Majore).

Teucrium Grosii Pau - Bocoia (Litt. rhiphaeo).

Sideritis foetens Clem. - Almería, Barranco del Caballar. (Regno Granatense).

Sideritis glauca Cav. - Oriola (Regno valentino), 1. clas. atque unico.

Sideritis Lacaitae F. Q. - Despeñaperros (Castella), 1. unico. Nepeta algeriensis De Noe-Alger.

Nepeta A puleji Uer. - Paterna (Baetica).

Dracocephalum Mairei Emb. - Atlante Medio.

Stachys recta L. - Riera d'Osor (Catalaunia).

Stachys Font-Queri Pau - Tetauen (Imp. Maroc.), 1. clas.

Salvia aegyptiaca L. - Torres de Alcalá (Littore rhiphaeo).

Salvia candelabrum Boiss. - Convento de las Nieves (Regno Granat.), 1. clas.

Salvia interrupta Schousb. - Xauen (Gomara. - Imp. Maroccanum). 
Salvia Mouretii Batt. et Pitard - Imp. Maroc. occid.

Salvia officinalis L. Ssp. lavandulitolia Vahl. var. vellerea Cuatr.

- Sierra Mágina. (Regno Giennense), 1. clas.

Salvia ceratophylloides Ard. - Inter Terreti et Stravorino (Calabria).

Salvia Barrelieri Et1.; S. inamoena Vahl - Ubrique (Baetica). Salvia viridis L. - Sigilla (Beni-Uriaguel, Imp. Maroc.)

\section{Solanaceae}

Atropa Belladona L. - Serra de 1'Almuçara (Catalaunia).

Triguera Osbeckii (L.) Willk. - Medina Sidonia (Baetica).

Hyoscyamus albus L. - Torredembarra (Catalaunia).

Physalis Alkekengi L. - San Guim (Catalaunia).

Solanum sodomaeum L. - S. Fernando (Baetica).

Datura Metel L. - Calafell (Catalaunia).

Datura Stramonium L. - S. Pere de Vilamajor (Catalaunia).

\section{Scrophulariaceae}

Celsia Barnadesii (Vah1) G. Don. var. mauritanica Pau - Yebel Malmusi (In littore rhiphaeo).

Celsia Faurei Murb. - Ktama (Atlante rhiphaeo).

Celsia simuata Cav. - S. Fernando (Baetica).

Linaria arenaria DC. - Morbihan.

Linaria Cavanillesii Chav. - Montgó, c. Dènia (Regno Valentino).

Linaria Clementei Haens. - Sierra de Mijas (Regno Granat.) Linaria Jolyi Batt. - Tetauen (Imp. Maroc.)

Linaria gobantesiana Coincy - Gobantes (Regno Granat.),

1. clas.

Linaria hirta (L.) Moench - Nerja (Regno Granat.)

Linaria Pelliceriana (L.) Mill. - Cap de Creus (Catalaunia).

Linaria rhiphatlantica F. Q. - Yebel Lerz (Atlante rhiphaeo),

1. clas.

Linaria tingitana Boiss. Reut. - Tetauen (Yebala). 
Antirrhinum chrysothales F. Q. - Bocoia (Littore rhiphaeo). Antirrhinum Charidemii Lge. Cabo de Gata (Regno Granat.),

1. clas. et unico.

Antirrhinum molle L. - Montsec (Pyr. Catalauniae). Antirrhinum valentinum F. Q. - Xeresa (Regno Valent.), 1. clas. Scrophularia sambucifolia L. - Alcalá de los Gazules (Baetica).

Scrophularia Scorodonia L. - Trevélez (Sierra Nevada).

Scrophularia villosiuscula Ėmb, et Maire. - Zarkat (Atlante rhiphaeo).

Digitalis purpurea L. var. mauritanica Humb. et Maire - Yebel Iguermalez (In Atlante rhiphaeo).

\section{Plantaginaceae}

Plantago Loeflingii L. - Miranda de Ebro (Castella vet.)

\section{Rubiaceae}

Callipeltis Cucullaria DC. - Targuist (Atlante rhiphaeo).

\section{Dipsacaceae}

Scabiosa stellata L. - Tobarra (Albacete).

Scabiosa rutifolia Vahl - E1 Araix (Imp. Maroc.).

\section{Campanulaceae}

Legousia conferta (Moench) Samp. - Porto (Lusitania). Campanula dichotoma L. - Estepona (Málaga).

\section{Compositae}

Phagnalon Caroli-Paui F. Q. - Badú (Atlante rhiphaeo). Phagnalon calycinum (Cav.) DC. - Mogador, 1. clas. 
Perralderia Paui F. Q. - In Monte Asrú (Imp. Maroc.)

Diotis maritima (L.). - Inter Ceuta et Tetauen (Imp. Maroc.) Anthemis maritima L. - Cádiz (Baetica).

Anthemis nobilis L. - Grazalema (Baetica).

Anthemis Bovéana Gay. - Ktama (Atlante rhiphaeo).

Antenuis temuisecta Ball - E1 Araix (Imp. Maroc.)

Ormenis multicaulis Br. B1. et Maire. - Kenitra (Imp. Maroc.)

Chrysanthemum viscosum Desf. - E1 Araix (Imp. Maroc.)

Glossopappus chrysanthemoides Kze. - Utrera (Baetica).

Leucanthemum paludosum (Poir.) Bonnet - Amekran, c. Axdir (Littore rhiphaeo).

Leucantemum demnatense Murb. - Ktama (Atlante rhiphaeo). Senecio leucanthemifolius Poir. - Tetauen (Yebala).

Senecio petraeus Boiss. et Reut. - Grazalema (Baetica), 1. clas. Arctotis calendulacea Wilıd. - Cádiz (Baetica).

Cirsium lepthophyllum (P. et F. Q.) F. Q.; Chamaepeuce leptophylla Pau et Font Quer. - Ad pedem montis Iguermalez (Atlante rhiphaeo), 1. clas.

Cirsium rhiphaeum (P. et F. Q.) F. Q. var. tetauensis F. Q. (Robustior, capitulis majoribus, dense aggregatis). - In Monte Gorgues, supra Tetauen (Imp. Maroc')

Cinara humilis I. - Ubrique (Baetica).

Cinara algarbiensis Coss. subsp. hystrix (Ball) F. Q.; C. Hystrix Ball - Inter Sok-et-Tnin et Targuist (Imp. Maroc.)

Onopordon disectum Murbek - E1 Araix (Imp. Maroc.)

Mariana eburnea (Coss. et Dur.) Pau - Aranjuez (Castella).

A mberboa muricata (L.) DC. - Torre del Mar, c. Málaga (Regno Granat.)

Centaurea algeriensis Coss. et Dur. -- S. Juan de las Minas, c. Melilla.

Centaurea Clementei Boiss. - Ronda (Regno Granat).

Centaurea diluta Ait. - Paterna (Baetica).

Centaurea leucantha Pourr. var. grandifolia F. Q - Frumentaria insula, 1. clas.

Centaurea pullaia I. - Tetauen (Yebala). 
Centaurea salmantica I. var. valdemorensis (Cut.) - Verdú (Catalaunia).

Cathamus dianius (Webb) Coincy - Ebuso.

Kentrophyllum rhiphaeum F. Q. et Pau. - Axdir (Littore rhiphaeo), 1. clas.

Catananche caerulea $\mathrm{I}_{4}$. - San Guim (Catalaunia).

Geropogon glaber I. - Tarifa (Baetica).

Scorzonera pygmaea Sibt. et S. - Tizzi Iffri (Atlante rhiphaeo). Hypochaeris saldensis Batt. - Bougie, Cap. Carbon (Afr. bor.) Taraxacum tomentosum Lge. - San Guim (Catalaunia).

Sonchus Mas-Guindalii Pau et Font Quer. - Bocoia (Litt. rhiphaeo).

Crepis tingitana (Salzm.) Ball; Crepis baetica I.ge. - Picacho de Alcalá de los Gazules (Baetica).

Andrvala maroccana Pau; Paua maroccanu Cab. - Cala Blanca, c. Melilla, 1. clas.

Barcinone, 3I decembris I930.

DR. P. FONT QUER

Botanicae Sect. Scient. Nat. Barc. Musei Curator

J. SANTAMARÍA Horti Magister
E. GROS

Botanicus Collector, horti assistens

Adresse: P. Font QuER. - Apartado 593. - BARCEI,ONA 
JUNTA DE CIENCIES NATURALS DE BARCELONA

\section{INDEX SEMINUM}

QUAE

HORTUS BOTANICUS MUSEI BARCINONENSIS SCIENT. NAT. MUTUA COMMUTATIONE OFFERT

1931
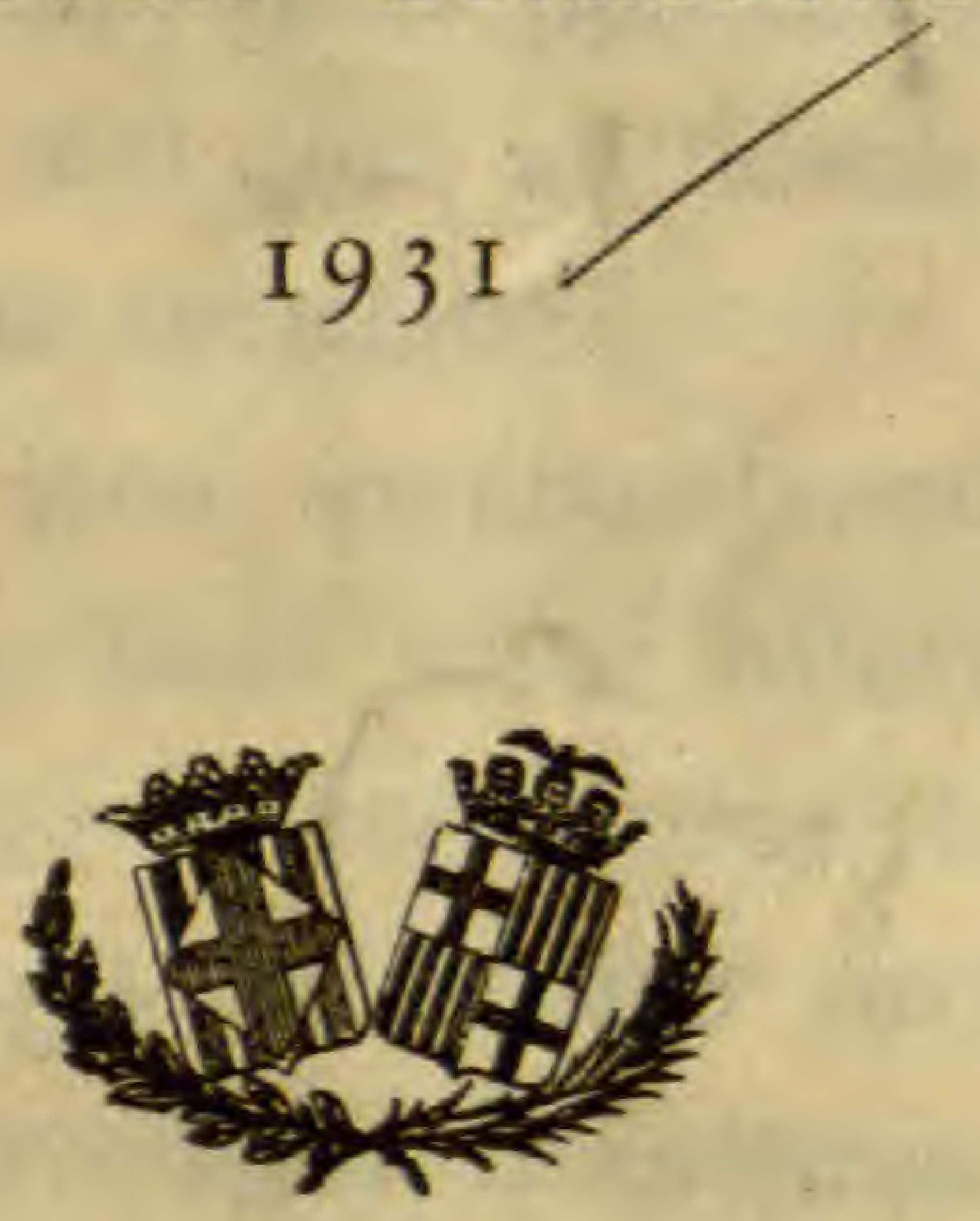

BARCINONE

TYPIS ELZEVIRIANIS 



\section{IN nostro hoc humili Viridario 1 Botanico Musei Barcinonensis nuper erecto, alias plantas colere nequimus nisi quas ad nostra stu- dia propius accedant. \\ Quare, loco magnae seminum varietatis, haec nunc offerimus, uniuscujusque indicantes locum originis, qui in nostro archivio fideliter invenitur inscriptus.}





\section{INDEX SEMINUM}

\section{QUAE HORTUS BOTANICUS MUSEI BARCINONENSIS SCIENT. NAT. MUTUA COMMUTATIONE OFFERT.}

\section{Juncaginaceae}

Triglochin laxiflora Guss. - Chiclana (Baetica).

\section{Gramineae}

Dactylis glomerata L. var. maritima Hack. - Ebuso, in parva insula Espardell de 1'Espartar dicta.

Festuca Font-Queri St.-Yv. - Tidiguin (Atlante rhiphaeo).

Oplismenus undulatifolius R. S. - Olot (Catalaunia).

\section{Araceae}

Biarum carratracense (Haens.) F. Q. - Carratraca (Regno Granat.), 1. clas.

Biarum tenuifolium (L.) Schott. - Benaocaz (Baetica).

\section{Liliaceae}

Merendera montana (L.) Lge. - Sierra Tejeda (Regno Granat.) Colchicum hisitanicum Brot. - Benaocaz.

Allium moschatum L. - San Guim (Catalaunia).

Allium nigrum L. - Atlante medio.

Allium pyrenaicum Costa et Vay. - Talaixà (Pyrenaeis), 1. clas. Allium stramineum Boiss. et Reut. var. xericiense P. Lara. -

Tarifa (Baetica).

Lilium Martagon L. - Montserrat.

Urginea undulata (Desf.) Stein. - Alcira (Regno Valent.)

Scilla obtusifolia Poir. - Salou (Catalaunia). 
Scilla Paui Lac. - Sierra de Alcaraz.

Scilla Ramburei Boiss. - Chiclana (Baetica).

Ornithogalum Reverchonii Lge. - Grazalema (Baetica).

Ornithogalum tenuifolium Guss. - Ebuso.

\section{Amaryllidaceae}

Leucojum autumnale L. - Despeñaperros (Castella Nova).

Narcissus dubius Gouan - Plana de S. Jordi (Catalaunia).

Narcissus poeticus L. - Montseny (Catalaunia).

Narcissus serotimus L. - Jerez de la Frontera (Baetica).

Narcissus elegans Spach var. fallax F. Q. - Bocoia (Litt. rhiphảeo).

Narcissus juncifolius Lag. - Montserrat.

Tapeinanthus humilis (Cav.) Herb. - Jerez.

\section{Iridaceae}

Iris alata Poir. - E1 Araix (Imp. Maroc.)

Iris Xiphium L. - La Barraca d'Aigües vives (Regno Valentino).

Iris tingitana Boiss. et Reut., vera. - R'gaia, c. Tandja, 1. clas. Gynandriris Sisyrinchium Parl. - Pto. de Sta. María (Baetica).

\section{Caryophyllaceae}

Minuartia maroccana Pau et F. Q. - Bocoia (Litt. rhiphaeo). Cerastium dichotomum L. - Targuist (Imp. Maroc).

Arenaria balearica L. - Puig Major (Insula Majore). Arenaria Pomelii Munby - Tizi Ifri, c. Targuist (Atlante rhiphaeo).

Arenaria spathulata Desf. - Yebel Malmusi (Bocoia - Littore rhiphaeo).

Silene disticha Willd. - Xauen (Gomara - Imp. maroccano). Silene glauca Pourr. - Hifac (Regno Valent.) 
Silene hifacensis Rouy - Ebuso.

Silene Cuatrecasasii Pau et Font Quer-Ktama (Atlante rhiphaeo).

Silene Otites L. - Estany d'Ibars (Catalaunia).

Silene psammitis Link - Inter Monda et Ojén (Regno Granat.) Lychnis coeli-rosa (L.) Desv. - Inter Esstepona et Casares (Regno Granat.)

Tunica Saxifraga (L.) Scop. var. grandiflora Goir. - E1 Chorro, c. Málaga (Regno Granat.)

Dianthus Seguieri Chaix - Girona (Catalaunia).

Dianthus catalaunicus Pourr. - Montseny.

Saponaria officinalis L. - Barcelona.

\section{Ranunculaceae}

Clematis campaniflora Brot. - Despeñaperros (Marianis montibus).

Ramunculus blepharicarpos Boiss. - Esparteros; c. Morón (Baetica).

Ranunculus sceleratus L. - Miranda de Ebro (Castella vet.) Thalictrum aquilegifolium L. - Montseny (Catalaunia).

Delphinium Requienii DC. - Insula Majore.

\section{Papaveraceae}

Papaver rupifragum Boiss. et Reut. - Grazalema (Baetica), 1. clas.

\section{Cruciferae}

Iberis gibraltarica L. - Tetauen (Imp. Maroc.) Thlaspi Prolongoi Boiss, - Grazalema (Baetica). Alyssum granatense Boiss. Reut. - Bu Meziat, c. Targuist (Atlante rhiphaeo). Alyssum spinosum L. - Sierra Nevada (Hispania). 
Psychine stylosa Desf. var. auriculata F. Q. - Axdir (Littore rhiphaeo).

Carrichtera anmua (L.) Prant1 - Frumentaria insula.

Succowia balearica L. - Insula Majore (Ba1.)

Vella psendocytisus L. - Aranjuez (Castella), 1. clas.

Vella spinosa Bois. - Dornajo (Sierra Nevada), 1. clas.

Sinapis alba L. - Albanchez (Regno Giennense).

Sinapis longirrostris Boiss. - Despeñaperros (Castella), 1. clas. Diplotaxis catholica (L.) D. C. var. bipinnatifida Kze. - Cádiz (Baetica).

Diplotaxis siifolia Kze. - S. Roque (Baetica), 1. clas.

Hemicrambe fruticulosa Webb - Tetauen (Imp. Maroc.), 1. clas. Coincya rupester Rouy - Alcaraz (Regno (Murcico), 1. clas. Draba hispanica L. - Dj. Lexhab (Imp. Maroc.)

Moricandia Ramburei Webb. - Algar (Baetica). Arabis alpina L. - Peña Mayor de Mena (Cantabria).

A rabis auriculata Lamk. - Tizi Ifri, c. Targuist (Atlante rhiphaeo).

A rabis parvula Duf. - Targist (Atlante rhiphaeo).

\section{Capparidaceae}

Capparis spinosa L. var. inermis Savi - Horta, pr. Barcelona.

\section{Saxifragaceae}

Saxifaga Vayredana Luiz. - Montseny.

\section{Rosaceae}

Crataegus laciniata Ucria - Ktama (Atlante rhiphaeo). Sanguisorba hybrida (L.) ; Poterium hybridum L., Sp. pl., ed. I, pág. 994; P. agrimonioides Spach - Los Términos, c. Grazalema (Baetica). 


\section{Leguminosae}

Genista biflora (Desf.) DC.; Spartium biflorum Desf. - Ebuso. Genista catalaunica (Webb) Rouy; Sarothammus catalannicus Webb-Montalegre, c. Barcelona, 1. clas.

Genista dorycniifolia F. Q. - Ebuso.

Genista Grosii F. Q.; G. dorycniifolia var. Grosii F. Q. - Ebuso. Genista equisetiformis Spach - Málaga (Regno Granatense).

Genista linifolia L. - Alcalá de los Gazules (Baetica).

Ononis reclinata L. - Montgó (Regno valentino).

- Ononis speciosa Lag. - Sierra de Cómpeta (Regno Granat.)

Trifolium atlanticum Ball - Zarkat (Atlante rhiphaeo).

Trifolium Tastetii F. Q. - Bu-Meziat, c. Targuist (Atlante rhiphaeo).

Medicago arborea L. var. citrina F. Q. - In parva insula Espartar dicta (Ëbuso).

Psoralea polystachya Poir. - El Araix (Imp. Maroc.)

Astragalus baeticus L. - S. Fernando (Baetica).

Astragatus lusitanicus Lam. - E1 Araix (Imp. Maroc.)

Biserrula Pelecinus L. - Málaga (Regno Granat.)

Scorpiurus vermiculata L. - Chiclana (Baetica).

Ornithopus compressus L. - Canillas de Albaida (Regno Granat.) Hippocrepis balearica Jacq. - Insula Majore.

Hippocrepis Salzmannii Boiss. Reut. - Sanlúcar de Barrameda (Baetica).

Onobrychis eriophora Desv. - Alcalá de los Gazules (Baetica). Onobrychis saxatilis Desv. - Sardanyola (Calalaunia).

Hedysarum pallidum Desf. - S. Juan de las Minas, pr. Melilla. Hedysarum flexuosum L. - R'gaia, c. Tandja.

Vicia Godronii Rouy. - Els Tres Salts, c. Manresa (Catalaunia). Vicia glauca Presl var. rerayensis Ball - Lexhab (GomaraImp. Maroc.)

Anthyllis hamosa Desf. - San Fernando (Baetica).

Anthyllis podocephala Boiss. - Sierra de Algodonales (Baētica). 


\section{Geraniaceae}

Erodium Sanguis-Christi Sennen - Ametlla, Plana de S. Tordi (Catalaunia), 1. clas.

\section{Zygophyllaceae}

Peganum Harmala L. - Chiprana (Aragonia).

Zygophyllum Fabago L. - Benahadux (Regno Granat.)

\section{Cneoraceae}

Cneorum tricoccum I. - Ebuso.

\section{Rutaceae}

Ruta montana L. - Targa (Catalaunia).

\section{Euphorbiaceae}

Euphorbia glebulosa Coss. - Yebel Malmusi (Littore rhiphaeo). Euphorbia rupicola Boiss. - Sierra Blanquilla, c. Cortes (Baetica).

Mercurialis serratifolia (Ball) Pau - Badú (Atlante rhiphaeo).

\section{Malvaceae}

Lavatera rotundata Laz. et Tub. - Aranjuez (Castella), 1. clas. Lavatera trimestris L. - Ubrique (Baetica).

\section{Guttiferae}

Hypericum balearicum L. - Insula Majore.

\section{Cistaceae}

Cistus Bourgeauanus Coss. - Chiclana (Baetica), 1. clas. Cistus populifolius L. var. lasiocalyx Willk. - Jimena de la Frontera (Baetica).

Halimium atriplicifolium Spach - Ankod (Gomara. - Imp. maroccano). 


\section{Violaceae}

Viola Demetria Prol. - Cerro de S. Cristóbal, c. Grazalema (Baetica).

\section{Onagraceae}

Onothera stricta Led, - La Línea (Baetica).

\section{Umbelliferae}

Eryngium glaciale Bois. - Tidiguin (Atlante rhiphaeo).

Tinguarra sicula (L.) Benth. et Hook. - Tetauen (Imp. Mar.)

Lagoecia cuminoides L. - Dos Hermanas (Baetica).

Bifora testiculata (L.) Spreng. - Ebuso.

Smyrnium perfoliatum L. - Güejar (Sierra Nevada).

A marintea trifida (Mill.) Samp.; Cachrys laevigata Lam. - Sierra

Nevada (Hispania).

Magydaris panacifolia Lge. - E1 Araix (Imp. Maroc.)

Heterothaenia thatictrifolia Boiss. - Benaocaz (Baetica).

Crithmum maritimum L. - Sitges (Catalaunia).

Angelica silvestris L. - Montseny (Catalaunia).

Capnophyllum peregrinum (L.) Lge. - R'gaia, c. Tandjá.

Ferula communis L. - Güejar (Sierra Nevada).

Peucedanum Cervaria L. - Girona (Catalaunia).

Pastinaca lucida Gouan - Aumallutx (Insula Majore).

Pastinaca silvestris Mill. - Tornabous (Catalaunia).

Thapsia garganica L. - Ebuso:

Thapsia villosa L. - Banyeres (Regno Valentino).

\section{Primulaceae}

Lysimachia minoriscensis Rodr. - Insula Minore.

\section{Plumbaginaceae}

Statice insignis Coss. - Benahadux (Regno Granat.) 


\section{Oleaceae}

Jasminum fruticans L. - Targa (Catalaunia).

\section{Asclepiadaceae}

Gomphocarpus fructicosus (L.) R. Br. - Barcelona.

\section{Convolvulaceae}

Convolvulus Vidalii Pau - Xauen (Imp. Maroc.), 1. clas.

\section{Borraginaceae}

Omphalodes brassicifolia (Lag.) Boiss. - Sierra Blanquilla, c. Cortes (Baetica).

Anchusa calcarea Boiss. - Veger (Baetica).

Anchusa sempervirens L. - Hoyos del Espino, in Sierra de Gredos (Hispania).

Cerinthe major L., a typica (Cerinthe flore rubro purpurascente, Bauh., Pin. 258, ex Linné). - S. Fernando (Baetica). Echium asturicum Lac. - Covadonga.

\section{Labiatae}

Ajuga Iva L. - Ebuso.

Teucrium bracteatum Desf. - Targuist (Atlante rhiphaeo).

Teucrium lancifolium Boiss. - Galatzó (Insula Majore).

Teucrium Grosii Pau - Bocoia (Litt. rhiphaeo).

Sideritis foetens Clem. - Almería, Barranco del Caballar. (Regno Granatense).

Sideritis glauca Cav. - Oriola (Regno valentino), 1. clas. atque unico.

Sideritis Lacaitae F. Q. - Despeñaperros (Castella), 1. unico. Nepeta A puleji Ucr. - Paterna (Baetica). Dracocephalum Mairei Emb. - Atlante Medio.

Stachys recta I. - Riera d'Osor (Catalaunia). 
Stachys Font-Queri Pau - Tetauen (Imp. Maroc.), 1. clas. Salvia aegyptiaca L. - Torres de Alcalá (Littore rhiphaeo). Salvia candelabrum Boiss. - Convento de las Nieves (Regno Granat,), 1. clas.

Salvia interrupta Schousb. - Xauen (Gomara. - Imp. Maroccanum).

Salvia Mouretii Batt. et Pitard - Imp. Maroc. occid.

Salvia officinalis L, ssp. lavandulifolia Vahl. var, vellerea Cuatr. - Sierra Mágina. (Regno Giennense), 1. clas.

Salvia ceratophylloides Ard. - Inter Terreti et Stravorino (Calabria).

Salvia Barrelieri Et1.; S. inamoena Vah1 - Ubrique (Baetica). Salvia viridis I. - Sigilla (Beni-Uriaguel, Imp. Maroc.)

\section{Solanaceae}

Atropa Belladona L. - Serra de 1'Almuçara (Catalaunia). Triguera Osbeckii (L.) Willk. - Medina Sidonia (Baetica). Hyoscyamus niger L. - Prades (Catalaunia). Hyoscyamus albus L. - Torredembarra (Catalaunia).

Physalis Alkekengi L. - San Guim (Catalaunia).

Withania somnifera Dun. - Saf-Saf, c. Melilla (Imp. Maroc.) Solanum sodomaeum L. - S. Fernando (Baetica).

Datura Metel L. - Calafell (Catalaunia).

Datura Stramonium L. - S. Pere de Vilamajor (Catalaunia).

\section{Scrophulariaceae}

Celsia Barnadesii (Vah1) G. Don. var. mauritanica Pau - Yebel Malmusi (In littore rhiphaeo).

Celsia Faurei Murb. - Ktama (Atlante rhiphaeo).

Celsia sinuata Cav. - S. Fernando (Baetica).

Linaria arenaria DC. - Morbihan.

Linaria Cavanillesii Chav.-Montgó, c. Dènia (Regno Valentino). Linaria Clementei Haens. - Sierra de Mijas (Regno Granat.) 
Linaria Jolyi Batt. - Tetauen (Imp. Maroc.)

Linaria gobantesiana Coincy - Gobantes (Regno Granat.), 1. clas. Linaria hirta (L.) Moench - Nerja (Regno Granat.) Linaria Pelliceriana (L.) Mill. - Cap de Creus (Catalaunia). Linaria rhiphatlantica F. Q. - Yebel Lerz (Atlante thiphaeo), 1. clas.

Linaria tingitana Boiss. Reut. - Tetauen (Yebala). Antirrhinum chrysothates F. Q. - Bocoia (Littore rhiphaeo). Antirrhinum Charidemii Lge. - Cabo de Gata (Regno Granat.), 1. clas. et unico.

Antirrhinum valentinum F. Q. - Xeresa (Regno Valent.), 1. clas. Scrophilaria sambucifolia L. - Alcalá de los Gazules (Baetica). Scrophularia Scorodonia L. - Trevélez (Sierra Nevada). Scrophularia villosiuscula Emb. et Maire. - Zarkat (Atlante rhiphaeo).

Digitalis purpurea L. var. mauritanica Humb. et Maire - Yebel Iguermalez (In Atlante rhiphaeo).

\section{Plantaginaceae}

Plantago Loeflingii L. - Miranda de Ebro (Castella vet.) Plantago notata Lag. E1 Zaio, c. Melilla (Imp. Maroc.)

\section{Rubiaceae}

Callipeltis Cucullaria DC. - Targuist (Atlante rhiphaeo).

\section{Dipsacaceae}

Scabiosa stellata L. - Tobarra (Albacete).

Scabiosa rutifolia Vah1 - E1 Araix (Imp. Maroc.).

\section{Campanulaceae}

Legousia conferta (Moench) Samp. - Porto (Lusitania). Campanula primulifolia Brot. - Cortegana (Huelva). 


\section{Compositae}

Phagnalon Caroli-Paui F. Q.-Badú (Atlante rhiphaeo). Phagnalon calycinum (Cav.) DC. - Mogador, 1. clas. Perralderia Paui F. Q. - In Monte Asrú (Imp. Maroc.) Diotis maritima (L.). - Inter Ceuta et Tetauen (Imp. Maroc.) Anthemis maritima L. - Cádiz (Baetica). Anthemis nobilis L. - Grazalema (Baetica). Anthemis Boveana Gay. - Ktama (Atlante rhiphaeo). Antemuis tenuisecta Ball - El Araix (Imp. Maroc.) Ormenis multicaulis Br. B1. et Maire. - Kenitra (Imp. Maroc.) Chrysanthemum viscosum Desf. - E1 Araix (Imp. Maroc.) Glossopappus chrysanthemoides Kze. - Utrera (Baetica). Leucanthemum paludosum (Poir.) Bonnet-Amekran, c. Axdir (Littore rhiphaeo).

Leucantemum demnatense Murb. - Ktama (Atlante rhiphaeo). Senecio lencanthemifolius Poir. - Tetauen (Yebala).

Senecio petraeus Boiss. et Reut. - Grazalema (Baetica), 1. clas. Arctotis calendulacea Willd. - Cádiz (Baetica).

Cirsium leptophyllum (P. et F. Q.) F., Q. ; Chamaepence leptophylla Pau et Font Quer. - Ad pedem montis Iguermalez (Atlante rhiphaeo), 1. clas.

Cirsium rhiphaeum (P. et F. Q.) F. Q. var. tetauensis F. Q. In "Monte Gorgues, supra Tetauen (Imp. Maroc.)

Cirsium rhiphceum F. Q. ssp. abylense (P. et F. Q.) F. Q. ;

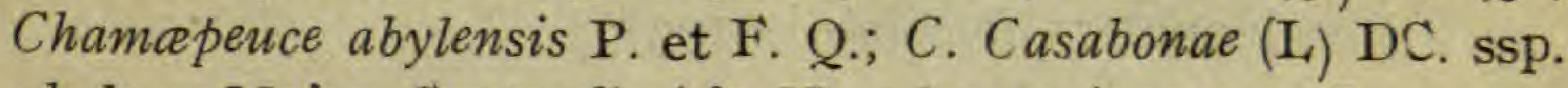
abylense Maire, Contr. f1. Afr. N. n. ${ }^{\circ}$ Io52 (I93I) - In monte Abyla, c. Ceuta (Imp. Maroc.).

Cinara humilis L. - Ubrique (Baetica).

Cinara algarbiensis Coss. subsp. hystrix (Ball) F. Q.; C. Hystrix Ball - Inter Sok-et-Tnin et Targuist (Imp. Maroc.) Onopordon dissectum Murbeck-E1 Araix (Imp. Maroc.) Mariana eburnea (Coss. et Dur.) Pau - Aranjuez (Castella). Amberboa muricata (L.) DC. - Torre del Mar, c. Málaga (Regno Granat.) 
Centaurea algeriensis Coss. et Dur. $-\mathrm{S}$. Juan de las Minas, c. Melilla.

Centaurea Clementei Boiss. - Ronda (Regno Granat.)

Centaurea diluta Ait. - Paterna (Baetica).

Centaurea leucantha Pourr. var. grandifolia F. Q. - Frumentaria insula, 1. clas.

Centaurea pullata L. - Tetauen (Yebala).

Centaurea salmantica L. var. valdemorensis (Cut.) - Verdú (Catalaunia).

Cathamus dianius (Webb) Coincy - Ebuso.

Rhaponticum acaule DC.-Monte Arruit, c. Melilla (Imp. Maroc.).

Kentrophyllum rhiphaeum F. Q. et Pau - Axdir (Littore rhiphaeo), 1. clas.

Catananche caerulea L. - San Guim (Catalaunia).

Geropogon glaber L. - Tarifa (Baetica).

Hypochaeris saldensis Batt. - Bougie, Cap. Carbon (Afr. bor.)

Taraxacum tomentosum Lge. - San Guim (Catalaunia).

Sonchus Mas-Guindalii Pau et Font Quer - Bocoia (Litt. rhiphaeo).

Crepis tingitana (Salzm.) Ball; Crepis baetica Lge. - Picacho de Alcalá de los Gazules (Baetica).

Andryala maroccana Pau; Paua maroccana Cab. - Cala Blanca,

c. Melilla, 1. clas.

Barcinone, 3I decembris I93I.

DR. P. FONT QUER

Botanicae Sect. Scient. Nat. Barc. Musei Curator

J. SANTAMaría

Horti Magister
E. Gros

Botanicus Collector, horti assistens

Adresse: P. FonT Quer. - Apartado 593. - BARCELONA 
JUNTA DE CIĖNCIES NATURALS DE BARCELONA

\section{INDEX SEMINUM}

QUAE

HORTUS BOTANICUS MUSEI BARCINONENSIS SCIENT. NAT. MUTUA COMMUTATIONE OFFERT

1932

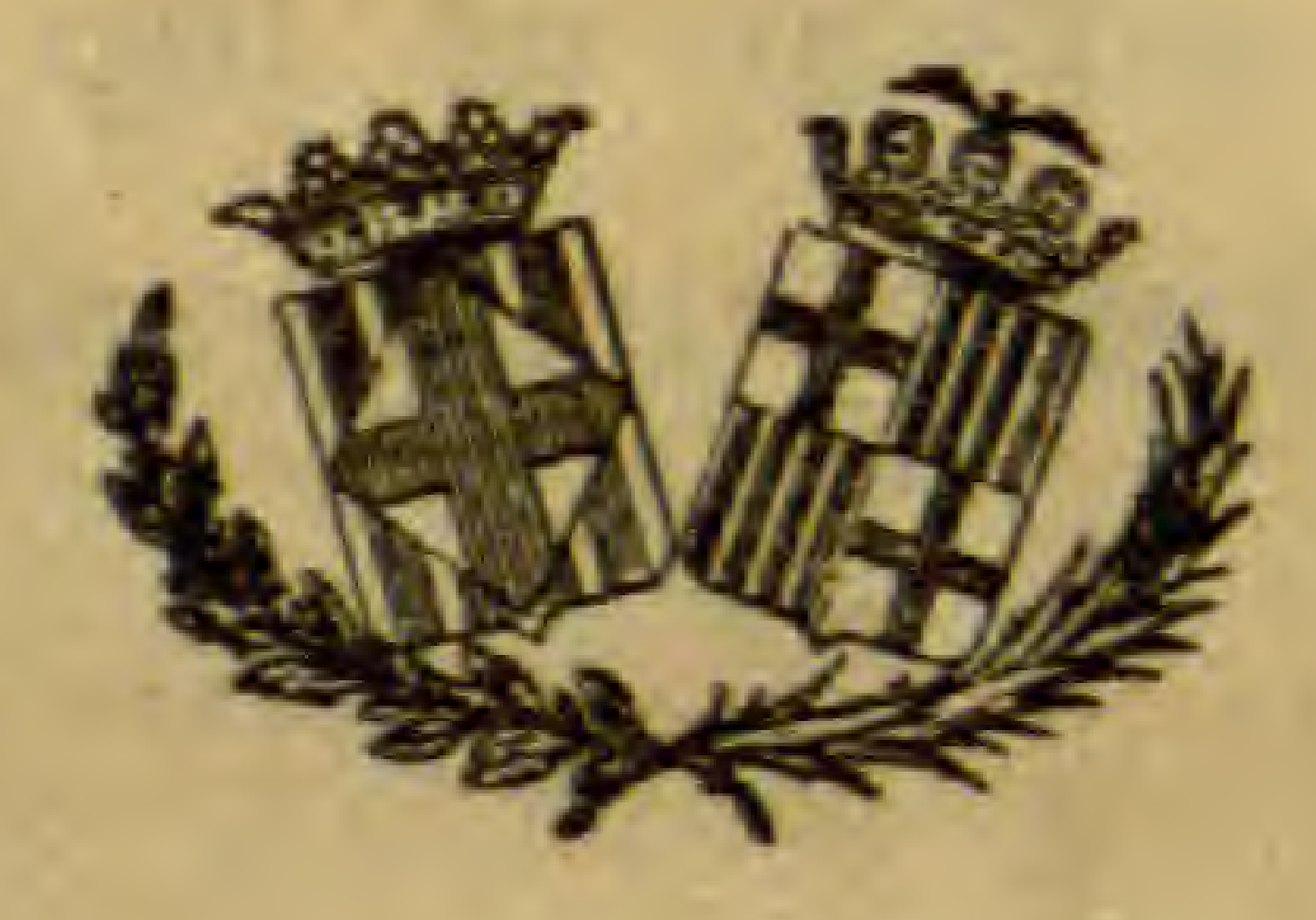

BARCINONE

TYPIS ELZEVIRIANIS

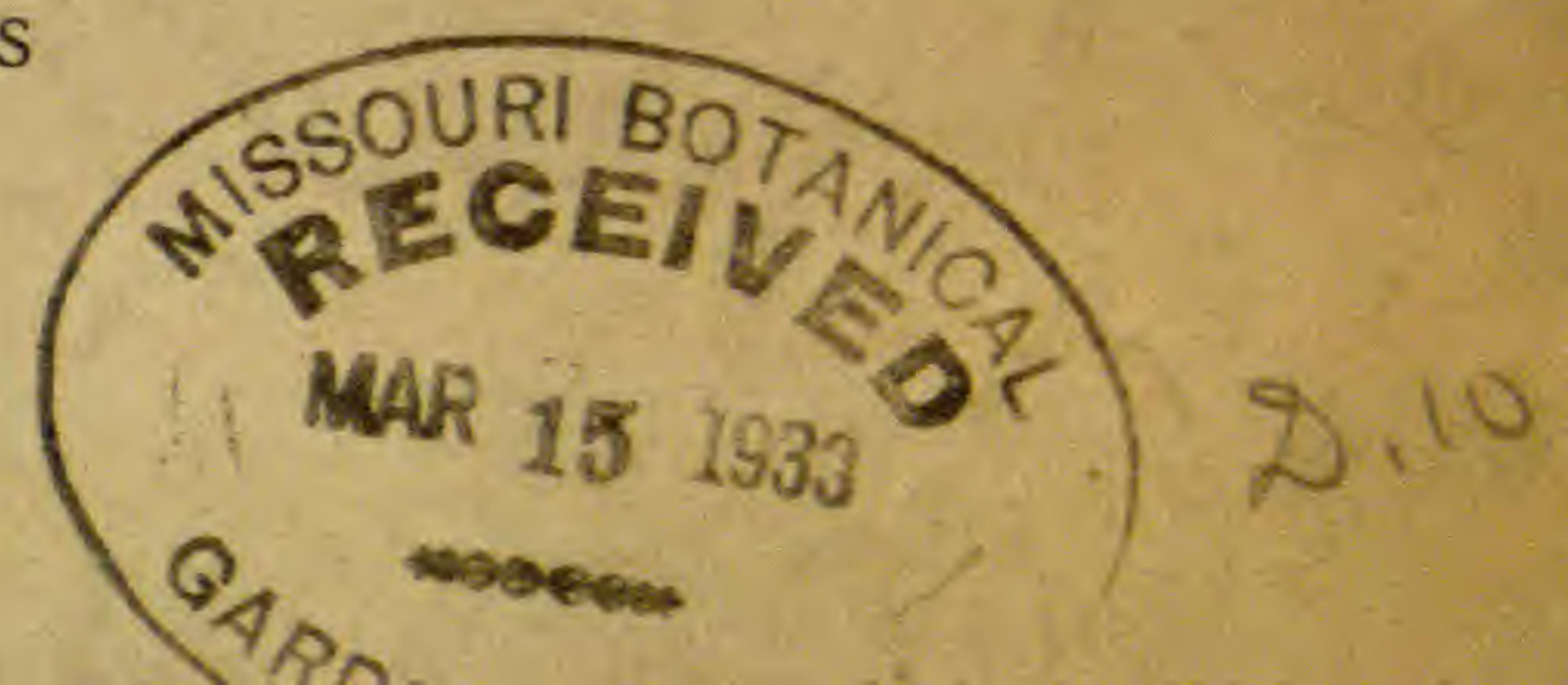


MUVINGS XACAI 
N nostro hoc humili Viridario
Botanico Musei Barcinonensis nuper erecto, alias plantas colere nequimus nisi quas ad nostra studia propius accedant.

Quare, loco magnae seminum varietatis, haec nunc offerimus, uniuscujusque indicantes locum originis, qui in nostro archivio fideliter invenitur inscriptus. 


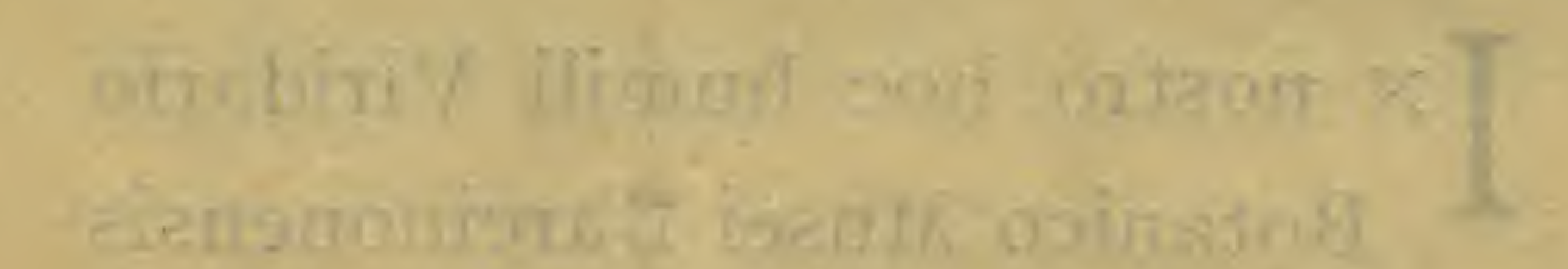

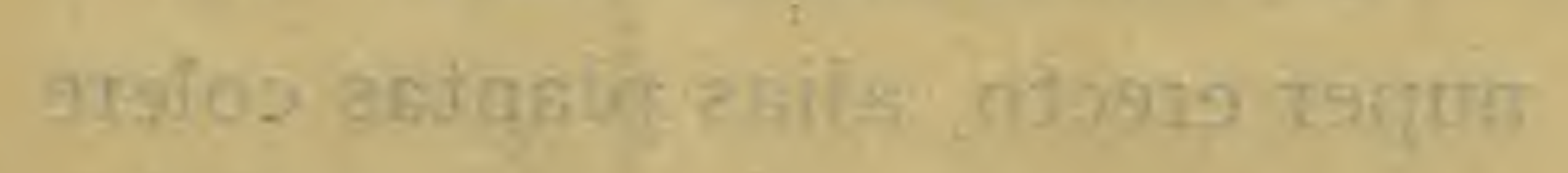

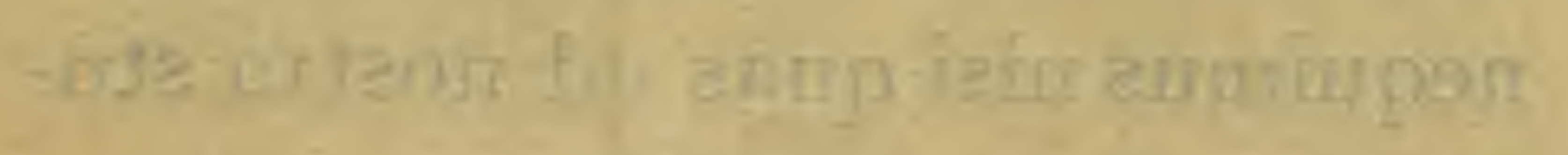

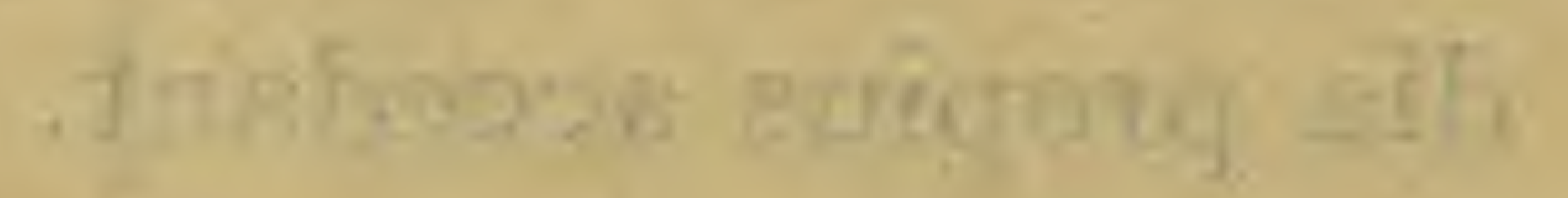
(m)

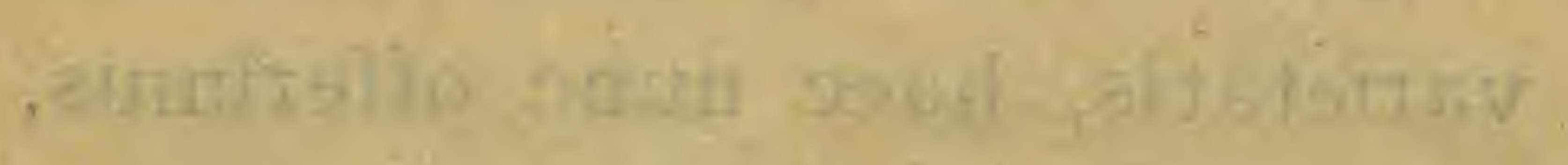

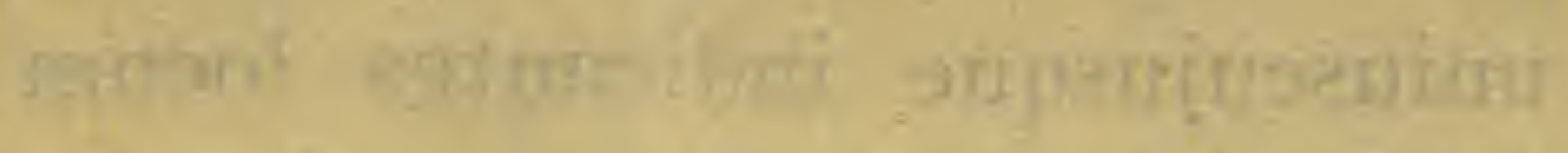

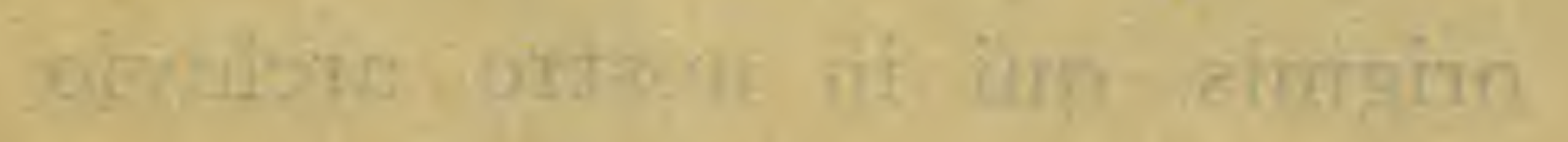

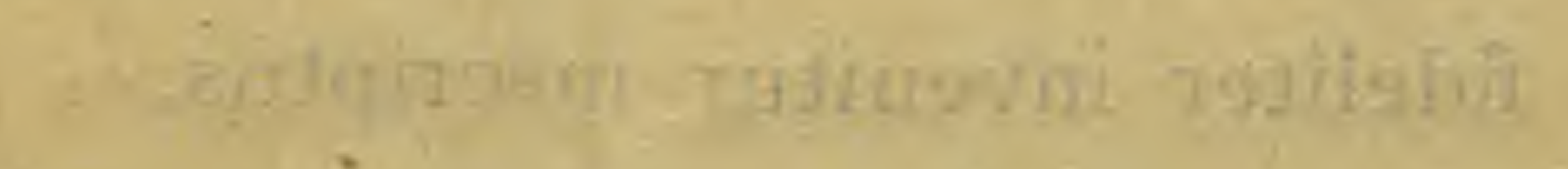




\section{INDEX SEMINUM}

QUAE HORTUS BOTANICUS MUSEI BARCINONENSIS SCIENT. NAT. MUTUA COMMUTATIONE OFFERT

\section{Juncaginaceae}

Triglochin laxiflora Guss. - Chiclana (Baetica).

\section{Gramineae}

Dactylis glomerata L. var. maritima Hack. - Ebuso, in parva insula Esspardell de 1'Espartar dicta.

Festuca Font-Queri St.-Yv. - Tidiguin (Atlante thiphaeo).

Oplismenus undulatifolius R. S. - Olot (Catalaunia).

\section{Araceae}

Biarum carratracense (Haens.) F. Q. - Carratraca (Regno Granat.), 1. clas.

Biarum temuifolium (L.) Schott. - Benaocaz (Baetica).

\section{Liliaceae}

Merendera montana (L.) Lge. - Sierra Tejeda (Regno Granat.) Colchicum lusitanicum Brot. - Benaocaz.

Allium moschatum L. - San Guim (Catalaunia).

Allium nigrum $\mathrm{L}$. - Atlante medio.

Allium pyrenaicum Costa et Vay. - Talaixà (Pyrenaeis), 1. clas.

Allium stramineum Boiss. et Reut. var. xericiense P. Lara -

Tarifa (Baetica).

Lilium Martagon L. - Montserrat.

Urginea undulata (Desf.) Stein. - Alcira (Regno Valent.)

Scilla obtusifolia Poir. - Salou (Catalaunia). 
Scilla Paui Lac. - Sierra de Alcaraz.

Scilla Ramburei Boiss. - Chiclana (Baetica).

Ornithogalum Reverchonii Lge. - Grazalema (Baetica).

\section{Amaryllidaceae}

Leucojum autumnale I. - Despeñaperros (Castella Nova).

Narcissus dubius Gouan. - Plana de S. Jordi (Catalaunia).

Narcissus poeticus L. - Montseny (Catalaunia).

Narcissus viridiflorus Schousb. - E1 Medik, inter Ceuta et Tetauen.

Narcissus serotinus I. - Jerez de la Frontera (Baetica).

Narcissus elegans Spach var. fallax F. Q. - Bocoia (Litt. rhiphaeo).

Narcissus juncifolius Lag. - Montserrat.

Tapeinanthus humilis (Cav.) Herb. - Jerez.

\section{Iridaceae}

Iris alata Poir. - E1 Araix (Imp. Maroc.)

Iris Xiphium L. - La Barraca d'Aigües vives (Regno Valentino).

Iris tingitana Boiss. et Reut., vera. - R'gaia, c. Tandja, 1. clas. Gynandriris Sisyrinchium Parl. - Pto. de Sta. María (Baetica).

\section{Caryophyllaceae}

Minuartia maroccana Pau et F. Q. - Bocoia (Litt. rhiphaeo). Cerastium perfoliatum $\mathrm{L}$. - Venta del Pino, Almería.

Cerastium dichotomum $\mathrm{L}$. - Targtiist (Imp. Maroc).

Arenaria balearica L. - Puig Major (Insula Majore).

Arenaria Pomelii Munby - Tizi Ifri, c. Targuist (Atlante rhiphaeo).

Arenaria spathulata Desf. - Yebel Malmusi (Bocoia - Littore rhiphaeo).

Silene disticha Willd. - Xatien (Gomara - Imp. maroccano). Silene glauca Pourr. - Hifac (Regno Valent.) 
Silene hifacensis Rouy - Ebuso.

Silene Cnatrecasasii Pau et Font Quer - Ktama (Atlante rhiphaeo).

Silene Otites I. - Esstany d'Ibars (Catalauinia).

Silene psammitis Link - Inter Monda et Ojén (Regno Granat.) Lychnis coeli-rosa (L.) Desv. - Inter Eistepona et Casares (Regno Granat.)

Tunica Saxifraga (L.) Scop. var. grandiflora Goir. - E1 Chorro, c. Málaga (Regno Granat.)

Dianthus Seguieri Chaix - Girona (Catalaunia).

Dianthus catalaunicus Pourr. - Montseny.

Saponaria officinalis $\mathrm{L}_{\mathrm{.}}-\rightarrow$ Barcelona.

\section{Ranunculaceae}

Clematis campaniflora Brot. - Despeñaperros (Marianis montibus).

Ranunculus blepharicarpos Boiss. - Essparteros, c. Morón (Baetica).

Ranunculus sceleratus L. - Miranda de Ebro (Castella vet.)

Thalictrum aquilegifolium L. - Montseny (Catalaunia).

Delphinium Requienii DC. - Insula Majore.

\section{Papaveraceae}

Papaver rupitragum Boiss. et Reut. - Grazalema (Baetica), 1. clas.

\section{Cruciferae}

Iberis Dunalii Cad. - Tossa (Catalaunia), 1. clas.

Iberis gibraltarica $\mathrm{I}_{\text {. }}$ - Tetauen (Imp. Maroc.)

Thlaspi Prolongoi Boiss. - Grazalema (Baetica).

Alyssum granatense Boiss. Reut. - Bu Meziat, c. Targuist (Atlante thiphaeo).

Alyssum spinosum L. - Sierra Nevada (Hispania).

Psychine stylosa Desf. var, auriculata F. Q. - Axdir (Littore rhiphaeo). 
Succorvia balearica L. - Insula Majore (Bal.)

Vella pseudocytisus I. - Aranjuez (Castella), 1. clas.

Vella spinosa Bois. - Dornajo (Sierra Nevada), 1. clas.

Sinapis alba L. - Albanchez (Regno Giennense).

Sinapis longirrostris Boiss. - Despeñaperros (Castella), 1. clas.

Diplotaxis catholica (L.) D. ,C. var. bipinnatifida Kze. - Cádiz

(Baetica).

Diplotaxis Siettiana Maire -. In parva insula Alboran, 1. clas. Diplotaxis siifolia Kze. - S. Roque (Baetica), 1. clas,

Hemicrambe fruticulosa Webb $\rightarrow$ Tetauen (Imp. Maroc.), 1. clas. Coincya rupester Rouy - Alcaraz (Regno Murcico), 1. clas. Draba hispanica L. - Dj. Lexhab (Imp. Maroc.)

Arabis alpina I. - Peña Mayor de Mena (Cantabria).

Arabis auriculata Lamk. - Tizi Ifri, c. Targuist (Atlante rhiphaeo).

Arabis parvula Duf. - Targist (Atlante rhiphaeo).

\section{Capparidaceae}

Capparis spinosa L. var. inermis Savi - Horta, pr. Barcelona.

\section{Saxifragaceae}

Saxifaga Vayredana Luiz. - Montseny.

\section{Rosaceae}

Crataegus laciniata Ucria - Ktama (Atlante rhiphaeo). Sanguisorba hybrida (I.) ; Poterium hybridum I., Sp. pl., ed. I, pág. 994; P. agrimonioides Spach - Los Términos, c. Grazalema (Baetica).

\section{Leguminosae}

Genista acutiflora Pau - E1 Araix, Imp. Maroc.; 1. clas. Genista biflora (Desf.) DC. ; Spartium biflorum Desf. - Ebuso. Genista catalaunica (Webb) Rouy; Sarothamnus catalaunicus

Webb - Montalegre, c. Barcelona, 1. clas. 
Genista dorycniifolia F. Q.-Ebuso.

Genista Grosii F. Q. ; G. dorycniifolia var. Grosii F. Q. - Ebuso. Genista equisetiformis Spach - Málaga (Regno Granatense).

Genista linifolia L. - Alcalá de los Gazules (Baetica).

Genista umbellata Poir. - Circa Axdir, I,ittore rhiphaeo.

Ononis reclinata $I_{1}$. - Montgó (Regno valentino).

Ononis speciosa Lag. - Sierra de Cómpeta (Regno Granat.)

Trifolium atlanticum Ball - Zarkat (Atlante rhiphaeo).

Trifolium Tastetii F. Q. - Bu-Meziat, c. Targuist (Atlante rhiphaeo).

Medicago arborea L. var. citrina F. Q. - In parva insula Espartar dicta (Ebuso).

Astragalus algarbiensis Coss. - E1 Araix, Imp. Maroc.

Astragalus baeticus L. - S. Fernando (Baetica).

Astragalus lusitanicus Lam.-- E1 Araix (Imp. Maroc.)

Biserrula Pelecinus L. - Málaga (Regno Granat.)

Scorpiurus vermiculata I. - Chiclana (Baetica).

Ornithopus compressus I. - Canillas de Albaida (Regno Granat.) Ornithopus durus Cav. - Veger (Baetica).

Hippocrepis balearica Jacq. - Insula Majore.

Hippocrepis Salzmannii Boiss. Reut. - Sanlúcar de Barrameda (Baetica).

Onobrychis eriophora Desv. - Alcalá de los Gazules (Baetica). Onobrychis saxatilis Desv: - Sardanyola (Catalaunia).

Hedysarum pallidum Desf. - S. Juan de las Minas, pr. Melilla. Hedysarum flexuosum I. -- R'gaia, c. Tandja.

Vicia Godronii Rouy. - Els Tres Salts, c. Manresa (Catalaunia). Vicia glauca Presl var. revayensis Ball - Lexhab (Gomara-

Imp. Maroc.)

Anthyllis hamosa Desf. - San Fernando (Baetica). Anthyllis podocephala Boiss. - Sierra de Algodonales (Baetica).

\section{Geraniaceae}

Erodium Sanguis-Christi Sennen - Ametlla, Plana de S. Jordi (Catalaunia), 1. clas. 


\section{Zygophyllaceae}

Peganum Harmala L. - Chiprana (Aragonia).

Zygophyllum Fabago L. - Benahadux (Regno Granat.)

\section{Cneoraceae}

Cneorum tricoccum L. - Ebuso.

\section{Rutaceae}

Ruta montana L. - Tàrrega (Catalaunia).

\section{Euphorbiaceae}

Euphorbia glebulosa Coss. - Yebel Malmusi (Littore thiphaeo). Euphorbia rupicola Boiss. - Sierra Blanquilla, c. Cortes (Baetica).

Mercurialis serratifolia (Ball) Pau - Badú (Atlante rhiphaeo).

\section{Malvaceae}

Lavatera rotundata $\mathrm{L}_{\mathrm{h}} \mathrm{z}$. et Tub. - Aranjuez (Castella), 1. clas. Lavatera trimestris L. - Ubrique (Baetica).

\section{Guttiferae}

Hypericum balearicum I. - Insula Majore.

\section{Cistaceae}

Cistus Bourgeauanus Coss. - Chiclana (Baetica), 1. clas.

Cistus ladaniferus L. fma. grandimaculatus. - Targuist, in Atlante rhiphaeo.

Cistus populifolius I. var. lasiocalyx Willk. - Jimena de la Frontera (Baetica).

Halimium atriplicifolium Spach - Ankod (Gomara. - Imp. maroccano).

\section{Violaceae}

Viola Demetria Prol. - Cerro de S. Cristóbal, c. Grazalema (Baetica).

\section{Onagraceae}

Onothera stricta Led. - La Línea (Baetica). 


\section{Umbelliferae}

Eryngium glaciale Bois. - Tidiguin (Atlante rhiphaeo).

Tinguarra sicula (L.) Benth. et Hook. - Tetauen (Imp. Mar.)

Lagoecia cuminoides L. - Dos Hermanas (Baetica).

Bifora testiculata (L.) Spreng. - Ebuso.

Smyrnium perfoliatum L. - Güejar (Sierra Nevada).

Amarintea trifida (Mill.) Samp. ; Cachrys laevigata Lam. - Sierra

Nevada (Hispania).

Magydaris panacifolia Lge. - E1 Araix (Imp. Maroc.)

Bupleurum montanum Coss. - Lexhab (Imp. Maroc.)

Bupleurum rigidum L. - Barcelona.

Heterothaenia thalictrifolia Boiss. - Benaocaz (Baetica).

Crithmum maritimum L. - Sitges (Catalaunia).

Seseli Vayredanum F. Q. - E1 Chorro (Málaga).

Angelica silvestris L. - Montseny (Catalaunia).

Capnophyllum peregrinum (L.) Lge. - R'gia, c. Tandja.

Ferula communis L. - Güejar (Sierra Nevada).

Peucedanum Cervaria I. - Girona (Catalaunia).

Pastinaca lucida Gouan - Aumallutx (Insula Majore).

Pastinaca silvestris Mill. - Tornabous (Catalaunia).

Thapsia garganica L. - Ebuso.

Thapsia villosa L. - Banyeres (Regno Valentino).

\section{Primulaceae}

Lysimachia minoriscensis Rodr. - Insula Minore.

\section{Plumbaginaceae}

Statice insignis Coss. - Benahadux (Regno Granat.)

\section{Oleaceae}

Jasminum fruticans I. - Tàrrega (Catalaunia).

\section{Asclepiadaceae}

Gomphocarpus fructicosus (L.) R. Br. - Barcelona. 


\section{Convolvulaceae}

Convolvulus Vidalii Pau - Xauen (Imp. Maroc.), 1. clas.

\section{Borraginaceae}

Omphalodes brassicitolia (Lag.) Boiss. - Sierra Blanquilla, c. Cortes (Baetica).

Anchusa calcarea Boiss. - Veger (Baetica).

Anchusa sempervirens I. - Hoyos del Espino, in Sierra de Gredos (Hispania).

Cerinthe major I., a typica (Cerinthe flore rubro purpurascente, Bauh., Pin. 258, ex Linné). - S. Fernando (Baetica). Echium asturicum Lac. - Covadonga.

\section{Labiatae}

Ajuga Iva L. - Ebusa.

Teucrium bracteatum Desf. - Targuist (Atlante rhiphaeo).

Teucrium lancifolium Boiss. - Galatzó (Insula Majore).

Teucrium Grosii Pau - Bacoia (Litt. rhiphaeo).

Sideritis foetens Clem. - Almería, Barranco del Caballar. (Regno Granatense).

Sideritis glauca Cav. - Oriola (Regno valentino), 1. clas. atque unico.

Sideritis Lacaitae F. Q. - Despeñaperros (Castella), 1. unico.

Nepeta Apuleji Ucr. - Paterna (Baetica).

Dracocephalum Mairei Emb. - Atlante Medio.

Stachys recta L. - Riera d'Osor (Catalaunia).

Stachys Font-Queri Pau - Tetauen (Imp. Maroc.), 1. clas.

Salvia aegyptiaca L. - Torres de Alcalá (Littore rhiphaeo).

Salvia candelabrum Boiss. - Convento de las Nieves (Regno Granat.), 1. clas.

Salvia interrupta Schousb. - Xatren (Gomara. - Imp. Maroccanum).

Salvia Mouretii Batt. et Pitard. - Imp. Maroc. occid. Salvia officinalis L. ssp. lavandulifolia Vahl, var. vellerea Cuatr. - Sierra Mágina. (Regno Giennense), 1. clas. 
Salvia ceratophylloides Ard. - Inter Terreti et Stravorino (Calabria).

Salvia Barrelieri Ett,; S. inamoena Vahl - Ubrique (Baetica). Salvia viridis L. - Sigilla (Beni-Uriaguel, Imp. Maroc.)

\section{Solanaceae}

Atropa Belladona L. - Serra de I'Almuçara (Catalaunia).

Triguera Osbeckii (L.) Willk, - Medina Sidonia (Baetica).

Hyoscyamus niger L. - Prades (Catalaunia).

Hyoscyamus albus L. - Torredembarra (Catalaunia).

Physalis Alkekengi L. - San Guim (Catalaunia).

Withania somnifera Dun. - Saf-Saf, c. Melilla (Imp. Maroc.)

Solanum sodomaeum I. - S. Fernando (Baetica).

Datura Metel L. - Calafell (Catalaunia).

Datura Stramonium L. - S. Pere de Vilamajor (Catalaunia).

\section{Scrophulariaceae}

Celsia Barnadesii (Vahl) G. Don. var. mauritanica Pau - Yebel

Malmusi (In littore rhiphaeo).

Celsia Faurei Murb. - Ktama (Atlante rhiphaeo).

Celsia sinuata Cav. - S. Fernando (Baetica).

Linaria arenaria DC. - Morbihan.

Linaria Cavanillesii Chav.-Montgó, c. Dènia (Regno Valentino). Linaria Clementei Haens. - Sierra de Mijas (Regno Granat.) Linaria Jolyi Batt. - Tetauen (Imp. Maroc.)

Linaria gobantesiana Coincy - Gobantes (Regno Granat.), 1. clas.

Linaria hirta (L.) Moench - Nerja (Regno Granat.)

Linaria Pelliceriana (I.) Mill. - Cap de Creus (Catalaunia). Linaria rhiphatlantica F, Q - Yebel Lerz (Atlante rhiphaeo),

1. clas.

Linaria tingitana Boiss. Reut. - Tetauen (Yebala). Antirrhinum Asarina L. - Montseny (Catalaunia). Antirrhinum chrysothales F. Q. - Bocoia (Littore rhiphaeo). 
Antirrhinum Charidemii Ige. - Cabo de Gata (Regno Granat.), 1. clas. et unico.

Antirrhinum valentinum F. Q. - Xeresa (Regno Valent.), 1. clas. Scrophularia sambucifolia $\mathrm{L}_{\text {. }}$ - Alcalá de los Gazules (Baetica). Scrophularia Scorodonia L. - Trevélez (Sierra Nevada).

Scrophularia villosiuscula Emb. et Maire - Zarkat (Atlante rhiphaeo).

Digitalis purpurea L. var. mauritanica Humb, et Maire - Yebel Iguermalez (In Atlante rhiphaeo).

\section{Plantaginaceae}

Plantago Loeflingii L. - Miranda de Ebro (Castella vet.) Plantago notata Lag. - E1 Zaio, c. Melilla (Imp. Maroc.) Plantago mauritanica Boiss. et Reut. - Beni Mhamed (Beni Zedjel - Imp. Maroc.)

\section{Rubiaceae}

Callipeltis Cucullaria DC. - Targuist (Atlante rhiphaeo).

\section{Dipsacaceae}

Scabiosa stellata L. - Tobarra (Albacete).

Scabiosa rutifolia Vah1-E1 Araix (Imp. Maroc.)

\section{Campanulaceae}

Legousia conferta (Moench) Samp. - Porto (Lusitania). Campanula afra Cav. - Estepona (Málaga).

Campanula primulifolia Brot. - Cortegana (Huelva).

\section{Compositae}

Phagnalon Caroli-Paui F. Q. - Badú (Atlante rhiphaeo). Phagnalon calycinum (Cav.) DC. - Mogador, 1. clas. Perralderia Paui F. Q. - In Monte Asrú (Imp. Maroc.) Diotis maritima (L.) - Inter Ceuta et Tetauen (Imp. Maroc.) Anthemis maritima L. - Cádiz (Baetica). 
Anthemis nobilis I. - Grazalęma (Baetica). Antenuis tenuisecta Ball - E1 Araix (Imp. Maroc.) Ormenis multicaulis Br. B1. et Mąire. - Kenitra (Imp. Maroc.) Chrysanthemum viscosum Desf. - E1 Araix (Imp. Maroc.) Glossopappus chrysanthemoides Kze. - Utrera (Baetica). Leucanthemum paludosum (Poir.) Bonnet - Amekran, c. Axdir (Littore rhiphaeo).

Leucantemum demnatense Murb. - Ktama (Atlante rhiphaeo). Senecio giganteus Desf. - Xauen, Imp. Maroc.

Senecio leucanthemifolius Poir. - Tetauen (Yebala).

Senecio leucophyllus DC. - Núria, Pyr. cat.

Senecio petraeus Boiss. et Reut. - Grazalema (Baetica), 1. clas. Senecio pyrenaicus Loefl.; S. Tournefortii Lap. - Núria, Pyr. Echinaps Font-Queri Pau - Xauen (Imp. Maroc.), 1. clas. Arctotis calendulacea Wilid. - Cádiz (Baetica). Cirsium leptophyllum (P. et F. Q.) F. Q.; Chamaepeuce leptophylla Pau et Font Quer. - Ad pedem montis Iguermalez (Atlante rhiphaeo), 1. clas.

Cirsium rhiphaeum (P. et F. Q.) F. Q. var. tetauensis F. Q. In Monte Gorgues, supra Tetauen (Imp. Maroc.) Cirsium rhiphaeum F. Q. ssp. abylense (P. et F. Q.) F. Q. ; Chamaepeuce abylensis P. et F. Q. ; C. Casabonae (L.) DC. ssp. abylense Maire, Contr. f1. Afr. N. n. ${ }^{0}$ I052 (I93I) - In monte Abyla, c. Ceuta (Imp. Maroc.)

Cardus granatensis Willk. var. tetuanensis Pau-Djebel Dersa, supra Tetauen, 1 . clas. Cinara humilis I. - Ubrique (Baetica).

Cinara algarbiensis Coss. subsp. hystrix (Ball) F. Q. ; C. Hystrix Ball - Inter Sok-et-Tnin et Targuist (Imp. Maroc.) Onopordon dissectum Murbeck - E1 Araix (Imp. Maroc.) * Mariana eburnea (Coss, et Dur.) Pau - Aranjuez (Castella). A mberboa muricata $\left(I_{\text {.. }}\right)$ DC. - Torre del Mar, c. Málaga (Regno Granat.)

Centaurea algeriensis Coss. et Dur. - S. Juan de las Minas, c. Melilla. 
Centaurea Clementei Boiss. - Ronda (Regno Granat.)

Centaurea diluta Ait. - Paterna (Baetica).

Centaurea leucantha Pourr. var. grandifolia F. Q. - Frumentaria insula, 1. clas.

Centaurea pullata L. $\rightarrow$ Tetauen (Yebala).

Centaurea salmantica L. var. valdemorensis (Cut.) - Verdú (Catalaunia).

Carthamus dianius (Webb) Coincy - Ebuso.

Rhaponticum acaule DC. - Monte Arruit, c. Melilla (Imp. Maroc.).

Kentrophyllum rhiphaeum F. Q. et Pau - Axdir (Littore rhiphaeo), 1. clas.

Catananche caexulea I. - San Guim (Catalaunia).

Geropogon glabex L. - Tarifa (Baetica).

Hypochaeris saldensis Batt:- Bougie, Cap. Carbon (Afr. bor.)

Taraxacum tomentosum Lge. - San Guim (Catalaunia).

Sonchus Mas-Guindalii Pau et Font Quer - Bocoia (Litt. thiphaeo).

Crepis tingitana (Salzm.) Ball ; Crepis baetica Lge. - Picacho de Alcalá de los Gazules (Baetica),

Andryala maroccana Pau; Pana maroccana Cab. - Cala Blanca, c. Melilla, 1. clas.

Barcinone, 3I decembris 1932 .

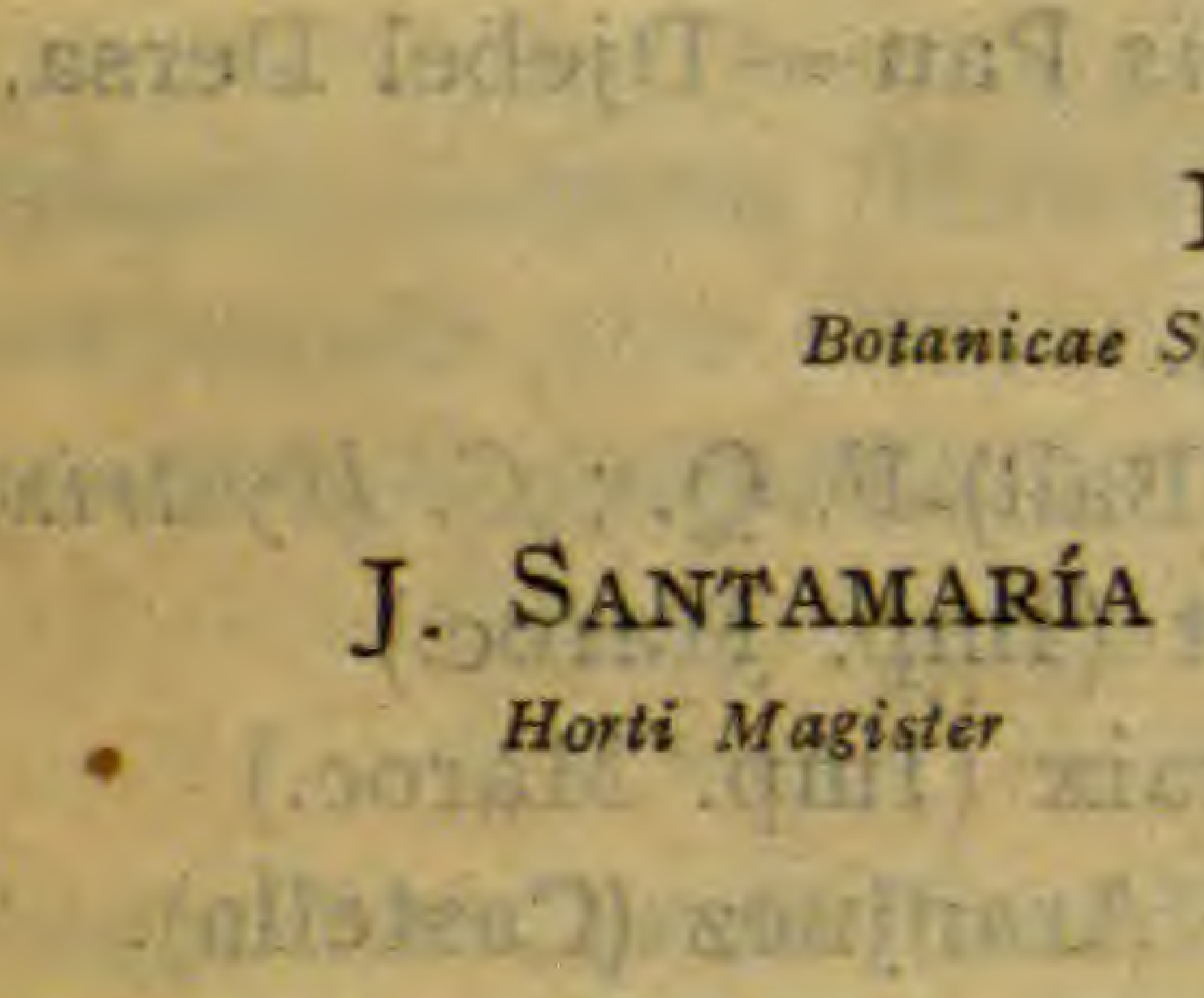

DR. P. FONT QUER Botanicae Sect: Scient. Nat, Barc, Musei Curator

Adresse: P. FONT QuER. - Apartado 593. - BARCELONA 
Missouri Botanias Sarden St. Lovis, ms., 4.8.a.

DIRECCIÓ TÈCNICA DELS SERVEIS BOTÀNICS DE CATA L U Y A

\section{INDEX SEMINUM} QUAE

HORTUS BOTANICUS BARCINONENSIS

\section{MUTUA COMMUTATIONE \\ OFFERT}

$$
1935^{2}
$$

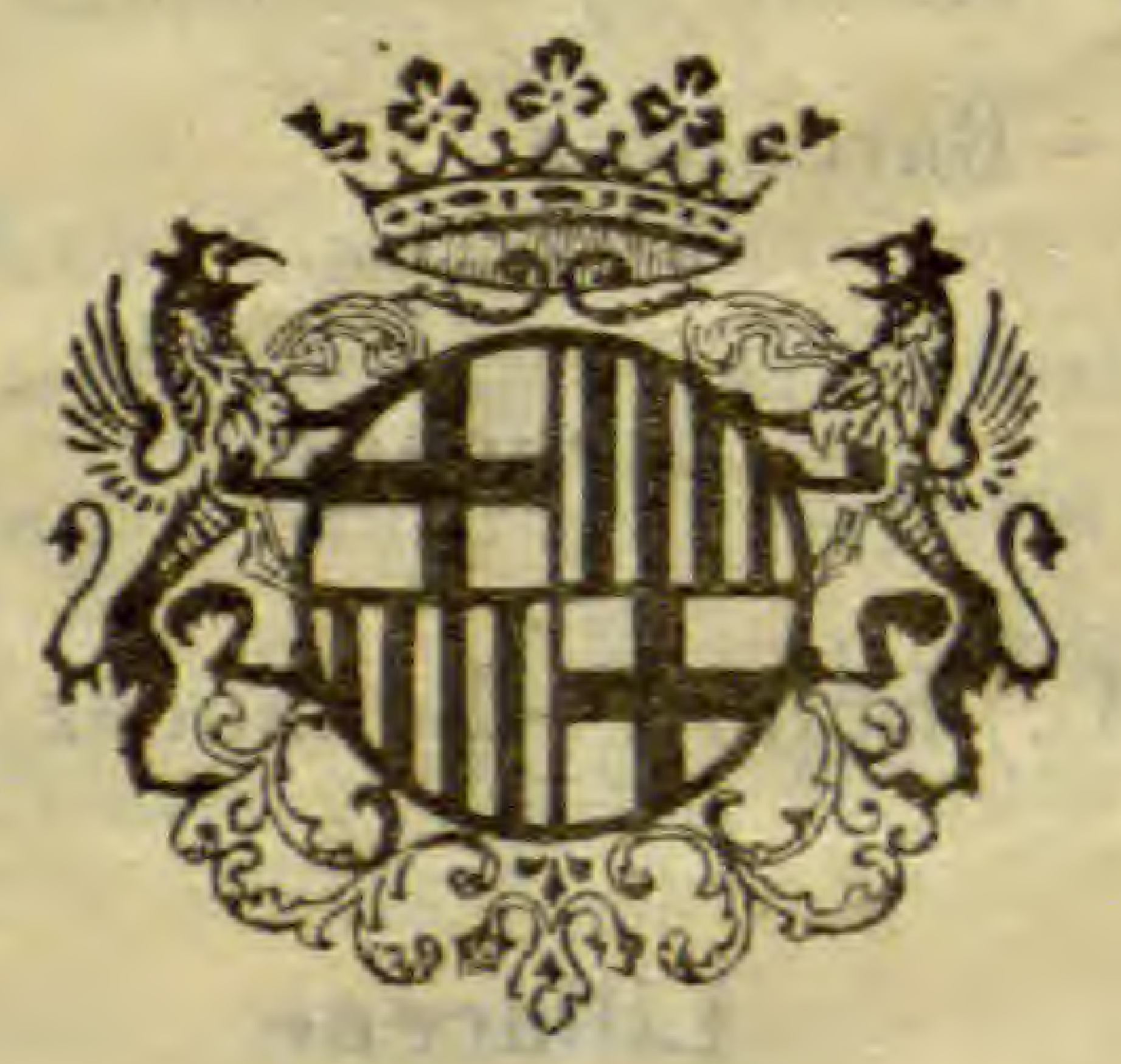

JUNTA DE CIÈNCIES NATURALS DE CATAIENYA

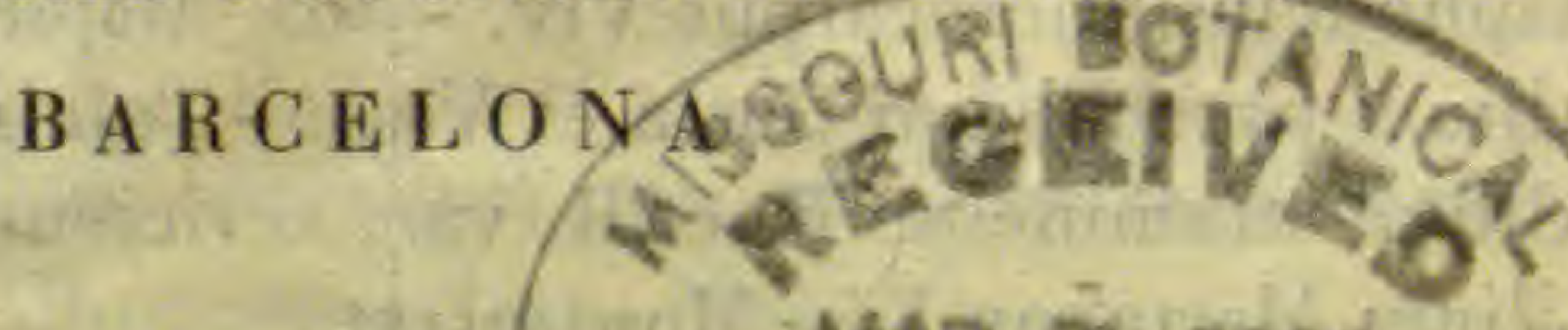

$$
\begin{aligned}
& \text { MAR } 77036
\end{aligned}
$$


Fritillaria Boissieri Costa-Montsant (Catalaunia).

Tulipa australis Link - Santa Elena (Baetica).

Urginea maritima (L.) Baker var. stenophylla Maire - Axdir, Bocoia (Imp. Maroc.).

Scilla hispanica Mill. - Lexhab (Imp. Maroc.).

Seilla Ramburei Boiss.-Chiclana (Baetica).

Scilla peruviana L. - Sierra de las Cabras (Baetica).

Scilla Paui Lac. - Sierra de Alcaraz, 1. clas.

Scilla obtusifolia Desf. - Plana de Sant Jordi (Catalaunia).

Ornithogalum sessiliflorum Desf. - Bocoia (Imp. Maroc.).

Ornithogalum narbonense L. - Borges Blanques (Catalaunia).

Ornithogalum Reverchonii Lge. - Grazalema (Baetica), 1. clas.

Muscari racemosum DC. - Alp (Pyren. catal.).

\section{Amaryllidaceae}

Leucojum autumnale L. - Despeñaperros, Sierra Morena (Baetica).

Tapeinanthus humilis Herbert - El Mimbral, Jerez (Baetica).

Narcissus serotinus L. - Jerez de la Frontera (Baetica).

Narcissus elegans (Haw.) Spach. var. fallax F. Q. - Morro Nuevo(Rif).

Narcissus elegans (Haw.) Spach. var. intermedius (Gay) Batt. Malalien, Tetuan (Imp. Maroc.).

Narcissus viridiflorus Schousb. - El Medik, inter Ceuta et Tetauen (Imp. Maroc.).

Narcissus papyraceus Ker.-Gawl. - Tetauen (Imp. Maroc.).

Narcissus dubius Gou. - Montroig (Catalaunia).

Narcissus juncifolius Lag. - Manresa; Girona (Catalaunia).

Narcissus monophyllus (Dur.). - Xauen (Imp. Maroc.).

Pancratium foetidum Pomel - Axdir, Bocoia (Imp. Maroc.).

\section{Iridaceae}

Iris juncea Poir. - El Medik, inter Ceuta et Tetauen (Imp. Maroc.). Iris pseudacorus L. - Sanaüja (Catalaunia).

Iris serotina Wk. - Dj. Tirira, Beni-Derkul (Imp. Maroc.).

Iris tingitana Boiss, et Reut, vera.-R'gaia, pr. Tandja, 1. clas.

Iris Fontanesii G. G. - Alhucemas (Imp. Maroc.). 
Dianthus catalaunicus Pourr. - Montseny (Catalaunia).

Saponaria officinalis L. - Barcelona.

Saponaria Vaccaria L. - Balaguer (Catalaunia occid.).

\section{Ranunculaceae}

Paeonia microcarpa Boiss. - Montsant (Catalaunia).

Helleborus foetidus L. - Prades (Catalaunia).

Helleborus trifolius Mill. ssp. lividus (Ait.) Briq. - Insula Cabrera (Balear.).

Delphinium Cossonianum Batt. - Xauia (Imp. Maroc.).

Delphinium Requienii DC.-Insula Majore (Bal.).

Delphinium Staphysagria L. - Ins. Formentera (Pityus.).

Clematis Vitalba L. - Santa Eulàlia de Ronçanes (Catalaunia).

Clematis campaniflora Brot.-Despeñaperros, Sierra Morena (Baet.). Ranunculus blepharicarpus Boiss. - Esparteros, c. Morón (Baetica).

Ranunculus sceleratus L. - Miranda de Ebro (Castella vet.).

Thalictrum aquilegifolium L. - Montseny (Catalaunia).

\section{Papaveraceae}

Chelidonium majus L. - Montseny (Catalaunia).

Glaucium flavum Crantz - Barcelona.

Roemeria hybrida DC. - Barcelona.

Papaver rupifragum B. et R. - Grazalema (Baetica), 1. clas.

Papaver rupifragum B. et R. var. atlanticum Ball - Atlante Majore, Papaver rupifragum $B$. et $R$. var. maroccanum F. Q. et Pau - Dj. Lexhab (Imp. Maroc.).

\section{Cruciferae}

Lepidium dhayense Munby - Tizi Ifri (Imp. Marec.).

Biscutella lyrata L. - Bab-Taza (Imp. Maroc.).

Ionopsidium Prolongoi Boiss. var. heterospermum (Batt.) Maire Lexhab (Imp. Maroc.).

Iberis gibraltarica L.-Tetauen, Beni Osmar (Imp. Maroc.).

Cakile maritima Scop. - Barcelona. 


\section{Rosaceae}

Crataegus laciniata Ucria - Ktama (Atlante rifeo).

Geum silvaticum Pourr. - Prades (Catalaunia).

Sanguisorba hybrida (L.) - Los Términos, pr. Grazalema (Baetica). Sanguisorba multicaulis (B. et R.) Asch. et Graebn. - Dj. Zem-Zem, inter Ceuta et Tetauen (Imp. Maroc.).

\section{Leguminosae}

Anagyris foetida L. - Ametrás (Imp. Maroc.).

Genista doryeniifolia F. Q. - Ebuso (Pityus.).

Genista equisetiformis Spach - Málaga (Baetica).

Genista linifolia L. - Alcalá de los Gazules (Baetica).

Retama monosperma (L.) Boiss. - Tánger (Imp. Maroc.).

Calycotome Grosii Pau et F. Q. - Axdir (Imp. Maroc.).

Ononis rosifolia DC. - Bab Taza (Imp. Maroc.).

Ononis speciosa Lag. - Sierra de Cómpeta (Baetica).

Ononis Maweana Ball - El Araix (Imp. Maroc.).

Ononis angustissima var, melillensis Maire - Uixan (Imp. Maroc.). Ononis arganietorum Maire - Talambot, pr. Xauen (Imp. Maroc.). Ononis reclinata L. - Montgó (Valentia); El Araix (Imp. Maroc.). Ononis mitissima L. - Targuist (Imp. Maroc.).

Ononis arborescens Desf. - Targuist (Imp. Maroc.).

Medicago arborea L. var. citrina F. Q. - In parva insula Espartar dicta (Pityus.).

Trifolium atlanticum Ball - Zarkat (Atlante rifeo).

Trifolium Tastetii F. Q. - Bu-Meziat, c. Targuist (Atlante rifeo).

Anthyllis fulgurans Porta - Insula Cabrera (Pitys.).

Anthyllis podocephala Boiss. - Sierra de Algodonales (Baetica).

Bonjeania hirsuta (L.) Rehb. - Barcelona.

Astragalus hamosus L. - Aitona (Catalaunia occid.).

Astragalus baeticus L. - San Fernando (Baetica).

Astragalus lusitanicus Lam. - El Araix (Imp. Maroc.).

Astragalus Font-Queri Maire et Sen. - Dj. Arruit (Rif or.), 1. clas. Scorpiurus vermiculata L. - Chiclana (Baetica).

Ornithopus compressus L. - Canillas de Albaida (Baetica). 


\section{Euphorbiaceae}

Chrozophora tinctoria (L.) Juss. - Barcelona.

Euphorbia pubescens Vaht - Melilla (Imp. Maroc.).

Euphorbia Lagascae Spr. - Almería.

Euphorbia glebulosa Coss. - Axdir (Imp. Maroc.).

Euphorbia rupicola Boiss. - Sierra Blanquilla, c. Cortes (Baetica).

Mercurialis Reverchonii Rouy - Badú (Atlante rifeo).

\section{Malvaceae}

Lavatera maritima Gouan - Axdir (Imp. Maroc.).

Lavatera trimestris L. - Ubrique (Baetica).

\section{Guttiferae}

Hypericum balearicum L. - Insula Majore (Bal.).

Hypericum montanum L. - Ktama (Atlante rifco).

Hyperieum Androsaemum L. - Montseny (Catalaunia).

\section{Cistaceae}

Cistus Libanotis L. (C. Clusii Dun.) - Almería, Venta del Pino (Baetica).

Cistus laurifolius L. - Prades (Catalaunia).

Cistus Bourgeauanus Coss. - Chiclana (Baetica), l. clas.

Cistus ladaniferus L. fma. grandimaculatus. - Targuist, in Atlante rifeo.

Cistus populifolius L. var. lasiocalyx Willk. -Jimena de la Frontera (Baetica).

Halimium atriplicifolium Spach - Ankod (Gomara, Imp. Maroc.).

\section{Onagraceae}

Onothera stricta Led, - La Linea (Bactiea). 


\section{Asclepiadaceae}

Gomphocarpus fruticosus (L.) R. Br. - Barcelona.

\section{Gentianaceae}

Chlora perfoliata L. - Barcelona.

\section{Convolvulaceae}

Convolvulus mauritanicus Boiss. - Xauen (Imp. Maroc.). Convolvulus Vidalii Pau - Xauen (Imp. Maroc.), 1. clas.

\section{Borraginaceae}

Heliotropium europaeum L. - Barcelona. Cynoglossum creticum Mill. - Barcelona. Anchusa calcarea Boiss. - Veger (Baetica). Anchusa sempervirens L. - Hoyos deI Espino (Sierra de Gredos). Cerinthe major L. var. typica - San Fernando (Baetica). Echium asturicum Lac. - Covadonga (Cantabria)

\section{Verbenaceae}

Verbena supina L. - Borges Blanques (Catalaunia occid.).

\section{Labiatae}

Ajuga Iva L. - Ebuso (Pityus.).

Teucrium Scorodonia L. - Fanals d'Aro (Catalaunia).

Teucrium lancifolium Boiss. - Galatzó (Insula Majore).

Teucrium flavum L. - Insula Majore (Balear.).

Lavandula Stoechas L. - Barcelona.

Lavandula latifolia Vill. - Barcelona, Castelldefels

Lavandula dentata L. - Axdir (Imp. Maroc.).

Lavandula maroccana Murb. - Atlante majore (Imp. Maroc.). 
Datura Metel L. - Calafell (Catalaunia).

Datura Stramonium L. - Sant Pere de Vilamajor (Catalaunia).

\section{Scrophulariaceae}

Verbascum Blattaria L. - La Cellera (Catalaunia).

Celsia Barnadesii (Vahl) G. Don var. mauritanica Pau - Axdir (In littore rifeo).

Celsia Faurei Murb. - Ktama (Atlante rifeo).

Celsia sinuata Cav. - San Fernando (Baetica).

Linaria arenaria DC. - Morbihan.

Linaria Cavanillesii Chav. - Montgó, c. Denia (Valentia).

Linaria melanantha B. et R. - Ateca (Aragonia).

Linaria gobantesiana Coincy - Gobantes (Baetica), 1. clas.

Antirrhinum Asarina L. - Montseny (Catalaunia).

Antirrhinum chrysothales F. Q. - Bocoia (Litt. rifeo).

Antirrhinum Charidemii Lge. - Cabo de Gata (Baetica), I. clas. et unic.

Antirrhinum hispanicum Chav. var. Jorroi Sen. - Tres Forcas, Melilla (Imp. Maroc.).

Antirrhinum Orontium L. - Ponferrada (León).

Chaenorrhinum rubrifolinm Lge. - Tizi Ifri, Rif (Imp. Maroc.).

Anarrhinum bellidifolium (L.) Desf. - Platja d'A ro (Catalaunia).

Scrophularia arguta Sol. - Atlante majore (Imp. Maroc.).

Scrophularia Scorodonia L. - Trevélez (Sierra Nevada).

Scrophularia sambucifolia L. - Alcalá de los Gazules (Baetica).

Scrophularia eriocalyx var. mauretanica Emb. et Maire - Dj. Iguermalez (Atl, rifeo).

\section{Plantaginaceae}

Plantago amplexicaulis Cav. - Almería.

Plantago ovata Forsk. - Cuevas, Almería (Baetica).

Plantago ovata Forsk. var. linearifolia - Sierra de Gador (Baetica).

Plantago Loeflingii L. - Miranda de Ebro (Castella vet.).

Plantago mauritanica B. et R. - Beni Mohamed, Beni Zedjel (Imp. Maroc.). 
Ormenis scariosa (Ball.) Lit. et Maire - Atlante majore.

Anacyclus radiatus Lois. - Tetauen (Imp. Maroc.).

Glossopappus chrysanthemoides Kze. - Utrera (Baetica).

Leucanthemum paludosum (Poir.) Bonnet - Amekran, c. Axdir (Litt, rifeo).

Leucanthemum osmariense (Ball) F. Q. - Gorgues, Beni Osmar (Imp. Maroc.), 1. clas.

Senecio Auricula Bourg. - La Codina (Catalaunia).

Calendula suffruticosa Vahl var. aestivalis Sen. - Kadia, Rif (Imp. Maroc.).

Echinops spinosus L. - Axdir (Rif centr.).

Echinops Font-Queri Pau - Xauen (Imp. Maroc.), 1. clas.

Arctotis calendulacea Willd - Cádiz (Baetica).

Arctium minus (Hill.) Bernh. - Barcelona.

Cirsium leptophyllum (P. et F. Q.) F. Q.; Chamaepeuce leptophylla

Pau et Font Quer - Ad pedem montis Iguermalez (Atlante rifeo), 1. clas.

Cirsium riphaeum (F. Q. et Pau) F. Q. - Ktama (Atlante rifeo).

Cirsium riphaeum (F. Q. et Pau) F. Q. var. tetauensis F. Q.- Gorgues, pr. Tetauen (Imp. Maroc.).

Cirsium riphaeum (F. Q. et Pau) F. Q. ssp. abylense (F. Q. et Pau)

F. Q. - In monte Abyla, c. Ceuta (Imp. Maroc.), I. unico.

Cirsium scabrum (Poir.) Bonnet et Barratte-El Araix (Imp. Maroc.).

Cnicus benedictus L. - Balaguer (Catalaunia).

Carduus granatensis Willk. var. tetuanensis Pau - Djebel Dersa, supra Tetauen, 1. clas.

Cinara humilis L. - Ubrique (Baetica).

Cinara algarbiensis Coss. subsp. Hystrix (Ball) F. Q.; C. Hystrix

Ball - Inter Sok-et-Tnin et Targuist (Imp. Maroc.).

Silybum eburneum Coss. et Dur. var. hispanicum Wk. - Aranjuez (Castella nova).

Onopordon dissectum Murbeck ssp. Murbeckii Lindb. var. lixense Maire - El Araix (Imp. Maroc.).

Amberboa muricata (L.) DC. - Torre del Mar, c. Málaga (Regno Granat.).

Centaurea sempervirens L. ssp. mauritanica F. Q. - Xaucn (Imp. Maroc.), 1. clas.

Centaurea pullata L. - Tetauen (Imp. Maroc.). 
Centaurea Clementei Boiss. - Ronda (Regno Granat.).

Centaurea diluta Ait. - Paterna (Baetica).

Centaurea algeriensis Coss. et Dur. - San Juan de las Minas, prope

Melilla (Imp. Maroc.).

Centaurea fragilis Dur. var. subinermis Pau - Melilla.

Centaurea dracunculifolia Duf. - Prat de Llobregat, pr. Barcelona.

Centaurea leucantha Pourr. var. grandifolia F. Q. - Ins. Formentera (Pityus.), 1. clas.

Carthamus riphaeus F. Q. - Snada, Rif (Imp. Maroc.).

Carthamus arborescens L. - Beni-Selman (Imp. Maroc.).

Carthamus dianius (Webb) Coincy - Ebuso (Pityus.).

Catananche lutea L. - Atlante majore.

Hypochaeris saldensis Batt. - Bougie, Cap Carbón (Afr. bor.).

Leontodon maroccanus (Pers.) Ball - El Araix (Imp. Maroc.).

Launaea cervicornis (Boiss.) F. Q. et Rothm. - Ins. Majore (Balear.).

Sonchus aquatilis Pourr, - Goles de l'Ebre (Catalaunia).

Sonchus Mas-Guindalii Pau et F. Q. - Bocoia (Litt, rifeo).

Lactuca cornigera F. Q. et Pau - Ktama (Imp. Maroc.).

Andryala mogadorensis Coss. et Bal. - Mogador (Imp. Maroc.).

Barcinone, 31 decembris 1935.

\section{Dr. P. FONT QUER}

\section{Horti Director}

W. ROTHMALER

Horti Assistens
J. SA N T A MARIA

Horti Magister

Adresse: P. Font Quer. - Apart. 593. - B A R C E L O N A 
INSTITUT BOTANIC DE BAREELONA

\section{INDEX SEMINUM QUAE}

HORTUS BOTANICUS BARCINONENSIS

$$
\begin{aligned}
& \text { MUTUA COMMUTATIONE } \\
& \text { OFFERT } \\
& \text { I } 9.37^{-}
\end{aligned}
$$

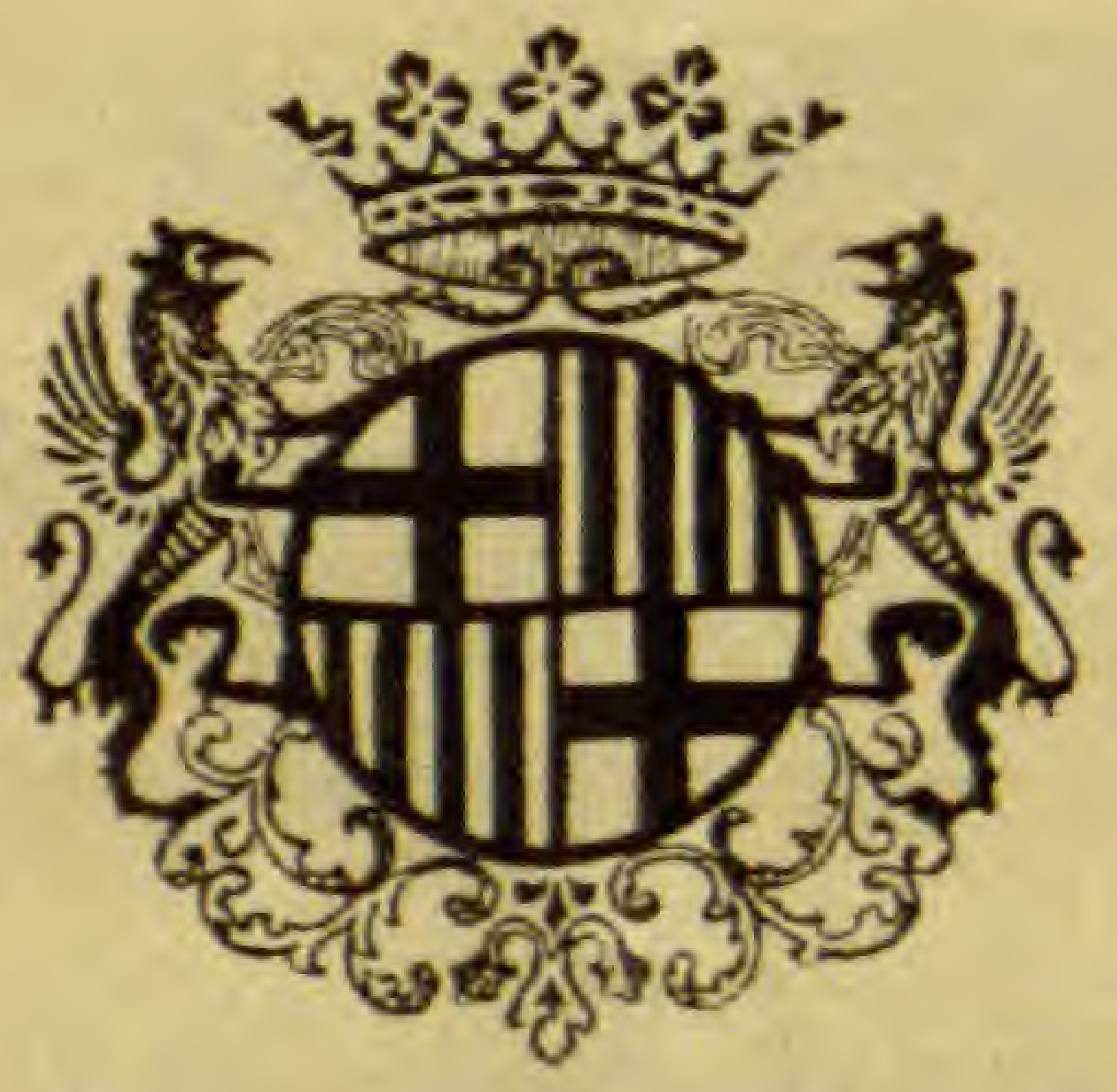

B A R C I N O XSOURI BOTAN/C EIVA 


\section{Cupressaceae}

Juniperus Oxycedrus L. - Barcelona.

\section{Juncaginaceae}

Triglochin Barrelieri Lois. var. maurum Pau-EI Medik inter Ceuta et Tetauen (Imp. Maroc.).

\section{Gramineae}

Paspalum dilatatum Poir. - Prat de Llobregat (Catal.).

Eleusine barcinonensis Costa-Barcelona.

Eremopyrum cristatum Led. - Balaguer (Catal.).

Stipa tenacissima L. - Ametlla de Mar (Catal.)

Avena sterilis L. - Tortosa.

Briza maxima L. - Platja d'Aro (Catalaunia).

Cynosurus obliquatus Link - Hauta-el-Kasdir (Imp. Maroc.).

\section{Cyperaceae}

Carex maxima Scop. - Barcelona.

\section{Liliaceae}

Merendera montana (L.) Lge.-Sierra Tejeda (Baetica).

Asphodelus cerasiferus Gay-Ports de Tortosa (Catalaunia).

Allium pyrenaicum Costa et Vayr. - Talaixà (Pyren.). 1. clas.

Allium stramineum B. et R. var. xericiense P. Lara-Tarifa (Baet.). Urginea maritima (L.) Baker var. stenophylla Maire - Axdir, Bocoia (Imp. Maroc.).

Scilla hispanica Mill. - Lexhab (Imp. Maroc.). 


\section{Polygonaceae}

Emex spinosa Campd. - Ins. Cabrera (Balear.).

Rumex tuberosus L.-Lexhab, pr. Xauen.

Rumex vesicarius L. var. rhodophysa Ball-Ifni.

\section{Chenopodiaceae}

Atriplex Halimus L. - Manresa (Catal.).

Bassia Reuteriana (Boiss.) Gürke - Lleida (Catalaunia), 1. clas.

\section{Caryophyllaceae}

Cerastium perfoliatum L. - Venta del Pino, Almería.

Cerastium dichotomum L. - Targuist (Imp. Maroc.).

Arenaria Pomelii Munby - Tizi Ifri, c. Targuist (Atlante rifeo).

Silene disticha Willd. - Xauen, Gomara (Imp. Maroc.).

Silene mollissima (L.) Pers.-Grazalema (Baetica).

Silene italica (L.) Pers. var. hesperia Maire - Ifni.

Silene hifacensis Rouy - Ebuso (Pityus.).

Silene Martyi Emb. et Maire - Bab-Taza (Imp. Maroc.).

Silene glauca Pourr. - Ins. Cabrera (Bal.).

Lychnis coeli-rosa (L.) Desv. - Inter Estepona et Casares (Baetica).

Melandryum macrocarpum Wk. - Serra Mariola (Val.).

Tunica Saxifraga (L.) Scop. var. grandiflora Goir. - El Chorro, c. Málaga.

Saponaria officinalis L. - Barcelona.

Saponaria Vaccaria L. - Balaguer (Catalaunia occid.).

\section{Ranunculaceae}

Paeonia microcarpa Boiss. - Montsant (Catalaunia).

Helleborus foetidus L. - Prades (Catalaunia).

Aquilegia nevadensis Bois. et Reut. var. Litardierei Senn. - Tidiguin (Atlante rifeo). 


\section{Resedaceae}

Reseda Luteola L. - Sierra Mágina (Baetica).

\section{Crassulaceae}

Kalanchoe Faustii F. Q. - Ifni.

\section{Rosaceae}

Geum silvaticum Pourr. - Prades (Catalaunia).

Sanguisorba hybrida (L.) - Los Términos, pr. Grazaleına (Baetica).

\section{Leguminosae}

Anagyris foetida (L.) - Ametrás (Imp. Maroc.).

Cytisus osmarensis (Coss.) Ball - In monte Gorgues, Beni Osmar,

1. clas. Arbusculum usque ad $5 \mathrm{~m}$ alt.

Genista dorycniifolia F. Q. - Ebuso (Pityus.).

Genista equisetiformis Spach-Málaga (Baetica).

Genista umbellata Poir. - Axdir (Rif).

Retama monosperma (L.) Boiss. - Tánger (Imp. Maroc.).

Ononis speciosa Lag. - Sierra de Cómpeta (Baetica).

Ononis angustissima var. melillensis Maire-Uixan (Imp. Maroc.). Ononis arganietorum Maire-Talambot, pr. Xauen (Imp. Maroc.). Ononis reclinata L. - Montgó (Valentia); El Araix (Imp. Maroc.). Ononis mitissima L. - Targuist (Imp. Maroc.).

Ononis arborescens Desf. - Targuist (Imp. Maroc.).

Medicago arborea L. var. citrina F. Q. - In parva insula Espartar dicta (Pityus.).

Astragalus hamosus L. - Aitona (Catalaunia occid.).

Astragalus baeticus L. - San Fernando (Baetica).

Astragalus lusitanicus Lam. - El Araix (Imp. Maroc.).

Astragalus Font-Queri Maire et Sen,-Dj. Arruit (Rif or.), I. clas. Astragalus akkensis Coss. - Ifni. 
Cistus Sennenianus Pau - Dj. Ker-ker, Rif, 1. clas.

Cistus ladaniferus L. fma. grandimaculatus - Targuist, in Atlante rifeo.

Fumana ericoides(Cav.)Pau var. glandulosa Pau - Almatret (Catal.). Halimium atriplicifolium Spach-Ankod (Gomara, Imp. Maroc.).

\section{Violaceae}

Viola catalonica W. Becker - El Pujolet, Barcelona, I. clas.

\section{Onagraceae}

Onothera stricta Led. - La Linea (Baetica).

\section{Umbelliferae}

Tinguarra sicula (L.) Benth. et Hook. - Tetauen (Imp. Maroc.).

Conium maculatum L. - Manresa (Catalaunia).

Magydaris panacifolia Lge. - El Araix (Imp. Maroc.).

Bupleurum protractum Hoff. et Lk. -Platja d'Aro (Catalaunia).

Bupleurum montanum Coss. - Lexhab (Imp. Maroc.).

Ammi Visnaga (L.) Lam. var. Hybernonis (Sen.) Maire - Tauima,

Rif or. (Imp. Maroc.).

Heterothaenia thalictrifolia Boiss. - Benaócaz (Baetica).

Spiroceratium Bicknellii (Briq.) Wolff - Ariant (Insula Majore),

1. clas.

Oenanthe callosa Salzm. - Zem-Zem, inter Ceuta et Tetauen

(Imp. Maroc.).

Foeniculum officinale All. - Sant Pere de Vilamajor (Catalaunia).

Ligusticum lucidum Mill. var. angustifolium Cuatrecasas - Sierra

Mágina (Jaén), 1. clas.

Capnophyllum peregrinum (L.) Lge. - R'gaia, c. Tandja (Imp. M.). Ferula communis L. - Güejar, Sierra Nevada (Baetica) et Ebuso (Pityus.). 
Sideritis grandiflora Salzm. - Tetauen (Imp. Maroc.).

Nepeta Nepetella (L.) Koch var. leucophylla Lge. - Alacant. 1. clas.

Nepeta Apuleji Ucr. - Paterna (Baetica).

Dracocephalum Mairei Emb. - Atlante Medio (Imp. Maroc.).

Phlomis Lychnitis L. - Serra Mariola (Valentia).

Phlomis maroccana Maire - Atlante Medio.

Phlomis Caballeroi Pau - Rif (Imp. Maroc.).

Stachys Fontqueri Pau - Tetauen (Imp. Maroc.), I. clas.

Salvia Sclarea L. var. hispanica Gav. - Gualba (Catalaunia).

Salvia Paui Maire - Xauen (Imp. Maroc.).

Salvia Barrelieri Etl. - Alcalá de los Gazules (Baetica); Zem-Zem (Imp. Maroc.).

Salvia Barrelieri Etl. var. pluripartita (Pau) Maire - El Araix (Imp. Maroc.).

Salvia algeriensis Desf. - Dar Kebdani; Xauia (Imp. Maroc.).

Salvia Mouretii Batt. et Pitard - Imp. Maroc. occid.

Salvia taraxacifolia Coss. - Atlante majore (Imp. Maroc.).

Salvia aegyptiaca L. - Beni Tuzin; Bocoia (Imp. Maroc.).

Hyssopus officinalis L. - Biosca (Catalaunia).

\section{Solanaceae}

Atropa Belladona L. - Serra d'Almuçara; Montserrat (Catalaunia). Atropa baetica Willk. - Sierra de la Nieve (Baetica).

Triguera Osbeckii (L.) Willk. - Medina Sidonia (Baetica).

Hyoscyamus niger L. - Prades (Catalaunia); Puerto Pajares (Astur.).

Hyoscyamus albus L. - Torredembarra (Catalaunia).

Physalis Alkekengi L. - Sant Guim (Catalaunia).

Solanum Sodomaeum L. - San Fernando (Baetica).

Datura Metel L. - Calafell (Catalaunia).

Datura Stramonium L. - Sant Pere de Vilamajor (Catalaunia). 


\section{Cucurbitaceae}

Bryonia dioica L. - Prades (Catalaunia).

Echallium Elaterium Rich. - Barcelona.

\section{. Campanulaceae}

Campanula affinis Roem. et Sch. - Montserrat, 1. clas.

\section{Compositae}

Phagnalon Caroli-Paui F. Q. - Badú (Atlante rifeo).

Phagnalon calycinum (Cav.) DC. - Mogador (Imp. Maroc.), I. clas. Perralderia Paui F. Q. - In Monte Asrú (Imp. Maroc.).

Anthemis Boveana Gay - Ktama (Atlante rifeo).

Anthemis maritima L. - Cádiz (Baetica).

Leucanthemum paludosum (Poir.) Bonnet - Amekran, c. Axdir

(Litt. rifeo).

Leucanthemum osmarense (Ball) F. Q. mutatio macrocephalum

- In H. B. Barc. ex sem. montis Beni Osmar producta.

Leucanthemum demnatense Murb. - Ktama (Atlante rifeo).

Calendula marginata Willd. - Gibraltar, I. clas.

Galendula suffruticosa Vahl var. aestivalis Sen. - Kadia, Rif (Imp.

Maroc.).

Echinops spinosus L. - Axdir (Rif centr.).

Echinops Font-Queri Pau - Xauen (Imp. Maroc.), 1. clas.

Cirsium leptophyllum (P. et F. Q.) F. Q.; Chamaepeuce leptophylla

Pau et Font Quer - Ad pedem montis Iguermalez (Atlante rifeo), 1. clas.

Cirsium riphaeum (F. Q. et Pau) F. Q. - Ktama (Atlante rifeo).

Cirsium riphaeum (F. Q, et Pau) F. Q. var. tetauensis F. Q. Gorgues, pr. Tetauen (Imp. Maroc.).

Cirsium riphaeum (F. Q. et Pau) F. Q. ssp. abylense (F. Q. et Pau)

F. Q. - In monte Abyla, c. Ceuta (Imp. Maroc.), 1. unico. 
Barcinonense, possibiliter vicinitate ratione polline parentum fecundata.

Carthamus dianius (Webb) Coincy - Ebuso (Pityus.).

Scorzonera pygmaea Sibth. et Sm. - Targuist (Atlante rifeo).

Leontodon maroccanus (Pers.) Ball - El Araix (Imp. Maroc.).

Launaea cervicornis (Boiss.) F. Q. et Rothm. - Ins. Majore (Balear.).

Sonchus aquatilis Pourr. - Goles de l'Ebre (Catalaunia).

Sonchus Mas-Guindalii Pau et F. Q. - Bocoia (Litt. rifeo).

Lactuca cornigera F. Q. et Pau - Ktama (Imp. Maroc.).

Andryala mogadorensis Coss. et Bal. - Mogador (Imp. Maroc.).

Crepis Triasii Willk. - Mallorea.

Barcinone, 31 decembris 1937.

DR. P. FONT QUER

Horti Director

J. SANT A MARIA

Horti Magister

Adresse: Instrtet Botànic, Carrer de S. Gervasi, 94.-BARCELONA 

INSTITUT BOTÀNIC DE BARCELONA

INDEX SEMINUM Q UAE

HORTUS BOTANICUS BARCINONENSIS
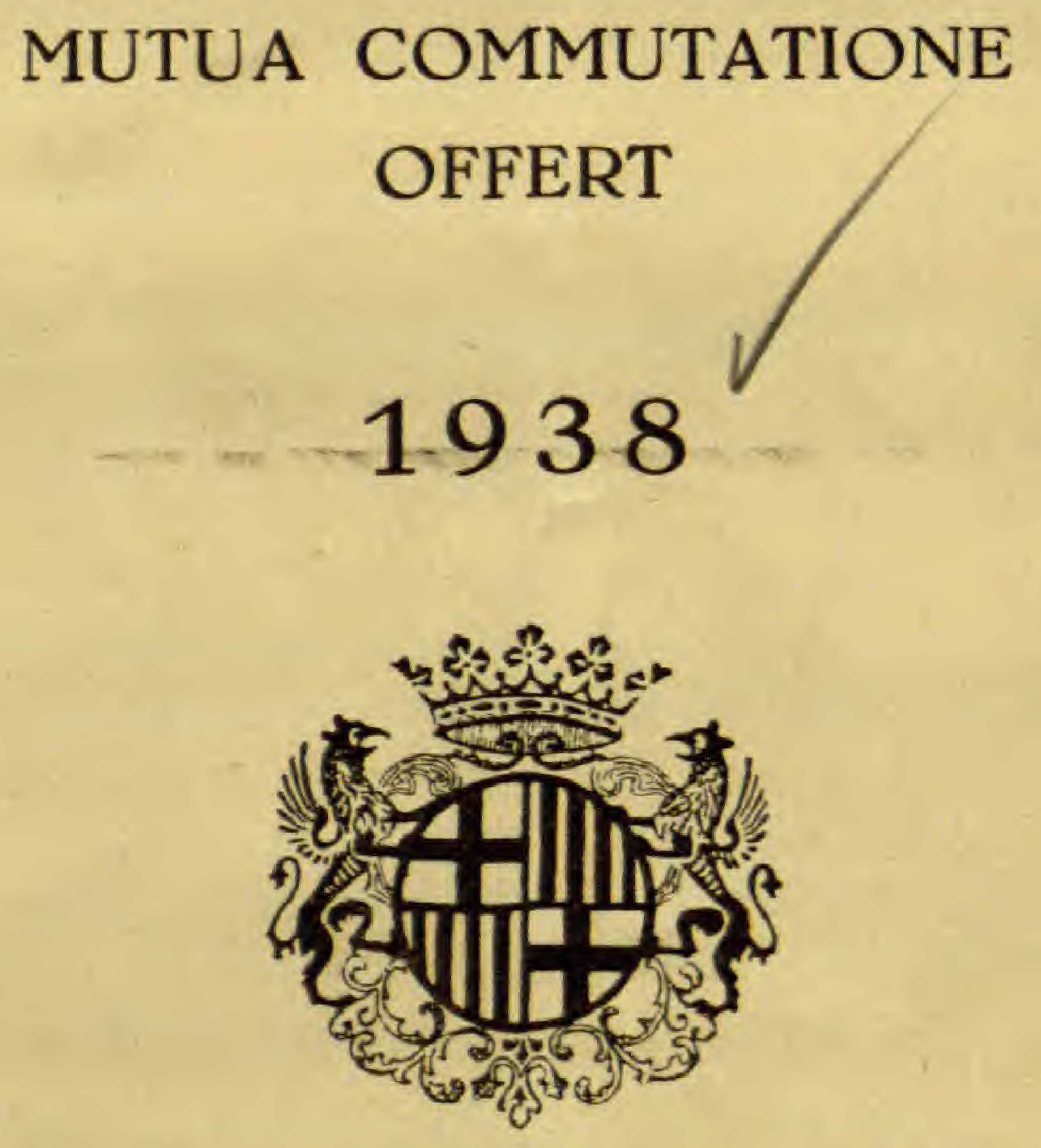

B A R C I N O N E 



\title{
P t e ridoph y t a
}

\author{
Polypodiaceae
}

Dawalia canariensis (L.) Smith-Baetica: Tarifa.

\section{Osmundaceae}

Osmunda regalis L.-Catalaunia: Montseny.

\section{Spermatoph y $\mathrm{t}$ a}

\section{Gymnospermae}

\section{Pinaceae}

Juniperus Oxycedrus L.-Barcelona: Castelldefels.

J. phoenicea L.-Barcelona: Castelldefels.

\section{Angiospermae}

\section{Monocotyledonea e}

\section{Juncaginaceae}

Triglochin Barrelieri Lois. var. maurum Pau-Mauritania: inter Ceuta et Tetauen.

T. laxiflorum Guss.-Baetica: Chiclana (Cádiz).

\section{Alismaceae}

Alisma Plantago-aquatica L.-Catalaunia: ad ripas fl. Ridaura. 


\section{Gramineae}

Ampelodesma mauritanicum (Poir.) Dur. et Schinz-Barcelona: Castelldefels.

Anthoxanthum odoratum L.-Catalaunia: Prades.

Avena sterilis L.-Catalaunia: Tortosa.

Briza maxima L.-Catalaunia: Platja d'Aro.

Coix Lacryma-Jovis L.-Frankfurt, ex horto botanico.

Cynosurus obliquatus Link -Mauritania: Hauta-el-kasdir (Beni Derkul).

Eatonia pennsylvanica A. Gray-Bruxelles, ex horto botanico.

Eleusine barcinonensis Costa-Barcelona.

Eremopyrum cristatum Led.-Catalaunia: Balaguer.

Oplismenus undulatifolius P. Beauv.-Catalaunia: Olot.

Pennisetum typhoideum Rich.-Castella: Ciudad Real, ubi colitur.

Phragmites Isiaca Kth.-Catalaunia: Calafell.

\section{Palmaceae}

Chamaerops humilis L.-Barcelona: Castelidefels.

\section{Cyperaceae}

Carex maxima L.-Barcelona.

\section{Liliaceae}

Allium pyrenaicum Costa et Vay.-Catalaunia: Talaixà, 1. clas.

A. scopulicolum F. Q.-Pithyusae: Ebuso, 1. clas.

A. stramineum Boiss. et Reut. var, xericiense P. Lara-Baetica: Tarifa. Asparagus horridus L.-Catalaunia: Tortosa.

Asphodelus acaulis Desf.-Mauritania: Melilla.

A. cerasiferus Gay-Catalaunia: Mont Caro, c. Tortosa.

A. microcarpus Viv.-Baleares: Calvia (Mallorca) et Menorca.

A. fistulosus L. fma. s, var. serrulatus Sennen-Mauritania: Kebdana (Rif. or.).

Hyacinthus amethystinus L.-Pyrenaei centrales.

Merendera montana (L.) Lge.-Baetica: Sierra Tejeda.

Muscari racemosum DC.-Pyrenaei or.: Alp.

Ornithogalum narbonense L.-Catalaunia: Les Borges Blanques.

O. Reverchonii Lge.-Baetica: Grazalema, 1. clas.

Phalangium algeriense Boiss. et Reut.-Mauritania: Tid-kid (Rif occid.). Polygonatum officinale All.-Catalaunia: Martorell de la Selva. 
Scilla hispanica Mill.-Mauritania: Dj. Lexhab, c. Bab Taza.

S. Paui Lac.-Murcia: Sierra de Alcaraz.

S. obtusifolia Desf.-Catalaunia: Plana de Sant Jordi.

S. Ramburei Boiss.-Baetica: Chiclana.

Tulipa australis Link-Baetica: Santa Elena, Sierra Morena.

Urginea maritima (L.) Baker - Pithyusae: Eivissa.

U. maritima (L.) Baker var. stenophylla Maire-Mauritania: Axdir (Rif centr.).

U. undulata (Desf.) Steinh.-Mauritania: El Araix.

\section{Amaryllidaceae}

Leucoium aestivum L. ssp. pulchellum (Salisb.) Briquet (Leucoium Hernandezii Camb., l, clas.).-Baleares: Menorca.

Narcissus Clusii Dun. (Corbularia monophylla Dur). - Mauritania: Xauen.

N. elegans (Haw.) Spach var. fallax F. Q.-Mauritania: Ras Sidi-elAhbed, c. Alhucemas.

N. elegans (Haw.) Spach var. intermedius Gay -Mauritania: Malalien, c. Tetauen.

N. Pseudo-Narcissus L.-Baetica: Sierra Mágina.

N. viridiflorus Schousb.-Mauritania: inter Ceuta et Tetauen.

Pancratium foetidum Pomel -Mauritania: Axdir (Rif centr.).

P. maritimum L.-Baleares: Menorca.

Tapeinanthus humilis (Cav.) Herb.-Baetica: Jerez de la Frontera.

\section{Iridaceae}

Iris Chamaeiris Bert.-Catalaunia: Ametlla de Mar.

I. Fontanesii G. G.-Mauritania: Axdir.

I. planifolia (Mill.) Fiori -Mauritania: El Araix.

L. Pseudacorus L.-Catalaunia: Sanaüja.

I. tingitana Boiss, et Reut.-R'gaia, c. Tandja, 1. clas.

\section{Orchidaceae}

Orchis longibracteata Biv.-Catalaunia: Platja d'Aro.

\section{Dicotyledonea e}

\section{Ulmaceae}

Celtis australis L.-Catalaunia. 


\section{Urticaceae}

Urtica dioica L.-Catalaunia: Montseny.

U. pilulifera L.-Mauritania: Tetauen.

\section{Aristolochiaceae}

Aristolochia Clematitis L.-Catalaunia: Castelló d’Empúries.

\section{Polygonaceae}

Emex spinosa Camdp.-Baleares: insula Cabrera. Rheum undulatum L.-Dresden, ex horto botanico. Rumex obtusifolius L.-Barcelona.

R. tuberosus L.-Mauritania: Dj. Lexhab, supra Bab Taza.

R. thyrsoides Desf.-Alger, ex horto botanico.

R. vesicarius L. var, rhodophysa Ball-Mauritania: Ifni.

\section{Chenopodiaceae}

Atriplex Halimus L.-Catalaunia: Manresa.

A. hortense L. var. rubrum Roth-Leyde, ex horto botanico.

\section{Amarantaceae}

Froelichia floridana (Nutt.) Moq.-Cluj, ex horto botanico.

\section{Nyctaginaceae}

Oxybaphus nyctagineus Sweet-Riga, ex horto botanico.

\section{Caryophyllaceae}

Arenaria Pomelii Munby -Mauritania: Tizi Ifri (Atlante rifeo).

A. serpillifolia L.-Pyrenaei centrales: Port de la Bonaigua.

Cerastium dichotomum L.-Mauritania: Targuist (Rif. centr.).

C. gibraltaricum Boiss. var. Boissieri (Gren.) Pau-Baetica: Sierra del Pinar.

C. perfoliatum L.-Baetica: Venta del Pino (Almería).

Cucubalus bacciferus L.-Coimbra, ex horto botanico.

Lychnis coeli-rosa (L.) Desv.-Baetica: inter Estepona et Casares.

Minuartia maroccana Pau et F. Q.-Mauritania: Axdir (Rif centr.). 
M. Sennenii Maire et Mauricio-Mauritania: Tres Forcas, c. Melilla, 1. clas.

Saponaria officinalis L.-Barcelona.

S. Vaccaria L.-Catalaunia: Balaguer.

Silene disticha Willd.-Mauritania: Xauen.

S. hifacensis Rouy -Pithyusae: Eivissa.

S. italica (L.) Pers, var. hesperia Maire-Mauritania: Ifni.

S. Martyi Emb. et Maire-Mauritania: Bab Taza.

S. mollissima (L.) Pers.-Baetica: Grazalema.

Tunica prolifera (L.) Scop,-Barcelona.

T. Saxifraga (L.) Scop. var. grandiflora Goir.-Baetica: El Chorro (Málaga).

\section{Ranunculaceae}

Clematis Vitalba L.-Catalaunia: Santa Eulàlia de Ronçana.

Delphinium Cossonianum Batt.-Mauritania: Xauia.

D. Requienii DC.-Baleares: Mallorca.

D. Staphysagria L.-Pithyusae: Formentera.

Helleborus foetidus L.- Catalaunia: Prades.

H. trifolius Mill. ssp. lividus (Ait.) Briq.-Baleares: insula Cabrera.

Paeonia microcarpa Boiss.-Catalaunia: Montsant.

Ranunculus blepharicarpos Boiss.-Baetica: Esparteros, c. Morón.

R. macrophyllus Desf.-Baleares: Menorca.

\section{Papaveraceae}

Argemone mexicana L.-Bucuresti, ex horto botanico.

Bocconia japonica André-Leyde, ex horto botanico.

Chelidonium majus L.-Catalaunia: Montseny.

Glaucium flavum Crantz - Barcelona.

Papaver rupifragum Boiss. et Reut.-Baetica: Grazalema, 1. clas.

P. rupifragum Boiss, et Reut. var. atlanticum Ball-Mauritania: Atlante majore.

P. rupifragum Boiss, et Reut. var. maroccanum F. Q. et Pau-Mauritania: Dj. Lexhab.

P. splendidissimum Boiss.-Wageningen, ex horto botanico.

\section{Cruciferae}

Alyssum spinosum L.-Baetica: Sierra Nevada.

Arabis alpina L.-Cantabria: Peña Mayor de Mena.

A. verna R. Br.-Mauritania: Bu-Meziat (Atlante rifeo). 
Barbarea praecox R. B.-Catalaunia: Martorell de la Selva.

Brassica balearica Pers.-Baleares: Mallorca.

Cheiranthus semperflorens Schousb.-Mauritania: Mogador.

Eruca sativa Lamk.-Catalaunia: Montfalcó, c. Cervera.

Hesperis matronalis L.-Frankfurt, ex horto botanico.

Ionopsidium Prolongoi (Boiss.) Batt. var. heterospermum (Batt.) Maire-Mauritania: Dj. Lexhab, c. Bab Taza.

Isatis tinctoria L.-Leyde, ex horto botanico.

I. Djurdjurae Coss.-Alger, ex horto botanico.

Lepidium latifolium L.-Leningrad, ex horto botanico.

Moricandia suffruticosa Desf. var. Philotei Sennen-Mauritania: Segangan (Rif or.).

Psychine stylosa Desf.-Mauritania: Axdir (Rif. centr.).

Sinapis alba L.-Baetica: Albanchez (Jaén).

Sisymbrium austriacum Jacq. var. rhedonense Degl. pro sp.-Legion:

Castillo de Cornatel, c. Ponferrada.

Succowia balearica L.-Baleares: Mallorca.

\section{Capparidaceae}

Capparis spinosa L. var. inermis Savi-Barcelona.

\section{Resedaceae}

Reseda lanceolata Lag.-Mauritania: Sok-el-Arbaa Taurirt (Rif. or.). R. Luteola L.-Baetica: Sierra Mágina.

\section{Crassulaceae}

Cotyledon Umbilicus-Veneris L.-Catalaunia: Gualba. Kalanchoe Faustii F. Q.-Mauritania: Ifni.

\section{Rosaceae}

Alchemilla mollis Buser-Kobenhavn, ex horto botanico.

Crataegus monogyna Jacq.-Barcelona.

Geum chilense Balb.-Chile: Concepción.

G. hispidum Fr.-Heidelberg, ex horto botanico.

G. silvaticum Pourr.-Catalaunia: Prades.

G. urbanum L.-Catalaunia: Montseny.

Sanguisorba hybrida (L.).-Baetica: Los Términos, c. Grazalema. 


\section{Leguminosae}

Amorpha fruticosa L.-Parma, ex horto botanico. Amphicarpa monoica Ell-Leyde, ex horto botanico. Anagyris foetida L.-Mauritania: Ametras (Beni Derkul). Anthyllis fulgurans Porta-Baleares: insula Cabrera. Astragalus akkensis Coss.-Mauritania: Ifni.

A baeticus L.-Baetica: San Fernando.

A. Font-Queri Maire et Sennen-Mauritania: Dj. Arruit (Rif or.), 1. clas.

A. hamosus L.-Catalaunia: Aitona.

A. Iusitanicus Lamk.-Mauritania: El Araix.

A. monspessulanus L. var. chlorocyaneus (Boiss. et Reut.) CostaBarcelona.

A. sesameus L.-Barcelona.

Calycotome Grosii Pau et F. Q.-Mauritania: Axdir (Rif centr.).

Cassia obovata Collad.-Catalaunia: Pineda, ubi colitur.

C. Sophera L.-Marseille, ex horto botanico.

Colutea arborescens L.-Barcelona.

Coronilla glauca L.-Mauritania: Dj. Gurugú, c. Melilla.

C. viminalis Salisbury - Mauritania: If ni.

Cytisus osmarensis (Coss.) Ball-Mauritania: Dj. Gorgues (Beni Osmar), 1. clas. Arbusculum usque ad $5 \mathrm{~m}$ alt.

Dalea Lagopus Willd.-Bucuresti, ex horto botanico.

Genista capitata (Cav.) Pau - Mauritania: El Araix.

G. dorycnifolia F. Q.-Pithyusae: Eivissa.

G. equisetiformis Spach-Baetica: Málaga.

G. umbellata Poir.-Mauritania: Axdir (Rif centr.).

Hippocrepis balearica Jacq. var, Grosii Pau-Pithyusae: Eivissa.

H. ciliata Willd.-Catalaunia: Tortosa.

H. Salzmannii Boiss. et Reut.-Baetica: Sanlúcar de Barrameda.

Lathyrus tingitanus L.-Baetica: Ubrique.

L. tingitanus L. var. coruscans (Maire) F. Q.-Mauritania: Bab Taza.

L. silvester L.-Barcelona.

Lupinus pilosus L. var. nitidus Emb.-Mauritania: Ifni.

Medicago arborea L. var. citrina F. Q.-Pithyusae: insula Espartar, juxta Ebuso.

M. Echinus DC.-Alger, ex horto botanico.

M. sphaerocephala Bert.-Catania, ex horto botanico.

Meibomia canadensis Kze.-Warszawa, ex horto botanico.

Onobrychis saxatilis Lamk.-Catalaunia: Cerdanyola.

Ononis arborescens Desf.-Mauritania: Targuist (Rif. centr.).

$\mathrm{O}$ arganietorum Maire-Mauritania: Talambot, c. Xauen. 
o. Chevalieri Sennen et Mauricio-Mauritania: Sok-el-Arbaa Taurirt (Rif. or.).

O. sicula Guss.-Mauritania: Mazuza (Rif or.).

Ornithopus compressus L.-Baetica: Canillas de Albaida.

Retama monosperma (L.) Boiss.-Mauritania: Tandja.

R. sphaerocarpa (L.) Boiss.-Catalaunia: Montagut, c. Lleida.

Segurigera Securidaca (L.) Deg. et Dorfl.-Alger, ex horto botanico.

Spartium junceum L.-Barcelona.

Trifolium angustifolium L.-Catalaunia: Santa Eulàlia de Ronçana.

Trigonella corniculata L.-Catania, ex horto botanico.

T. Foenum-graecum L.-Pisa, ex horto botanico.

Vicia Godronii Rouy.-Catalaunia: Manresa.

\section{Geraniaceae}

Erodium guttatum Willd.-Mauritania: Drius (Rif. or.).

\section{Linaceae}

Linum usitatissimum L.-Mauritania, ubi colitur.

\section{Zygophyllaceae}

Zygophyllum Fabago L.-Baetica: Benahadux (Almería).

\section{Cneoraceae}

Cneorum tricoccum L.-Pithyusae: Eivissa.

\section{Rutaceae}

Ruta bracteosa DC.-Barcelona, subspontanea.

\section{Euphorbiaceae}

Euphorbia Lagascae Spr.-Baeiica: Almería.

E. Lathyris L.-Parma, ex horto botanico.

E. Nereidum Jahandiez et Maire-Mauritania: Atlante majore. Mercurialis Reverchonii Rouy-Mauritania: Badú (Atlante rifeo).

\section{Anacardiaceae}

Pistacia Lentiscus L.-Barcelona. 
Aquifoliaceae

Ilex Aquifolium L.-Catalaunia: Sant Sadurni d'Osormort.

\section{Rhamnaceae}

Rhamnus Alaternus L.-Barcelona.

R. lycioides L.-Barcelona: Castelldefels.

\section{Malvaceae}

Anoda lavaterioides Medik.-Koenigsberg, ex horto botanico.

Lavatera maritima Gouan-Mauritania: Axdir (Rif. centr.).

L. micans L.-Aragonia: Mallén.

L. Olbia L. var. hispida (Desf.) G. G.-Alger, ex horto botanico.

L. thuringiaca L.-Frankfurt, ex horto botanico.

L. trimestris L.-Mauritania: Samsa, c. Tetauen.

Malachra capitata L.-Bucuresti, ex horto botanico.

Sida Napaea Cav.-Bruxelles, ex horto botanico.

\section{Sterculiaceae}

Melochia corchorifolia L.-Bucuresti, ex horto botanico.

\section{Guttiferae}

H. Androsaemum L.-Catalaunia: Montseny. Hypericum balearicum L.-Baleares: Mallorca.

H. montanum L.-Mauritania: Ktama (Atlante rifeo).

\section{Cistaceae}

Cistus albidus L.-Barcelona.

C. Bourgeauanus Coss.-Baetica: Chiclana.

C. hirsutus Lamk.-Coimbra, ex horto botanico.

C. ladaniferus L. var. petiolatus Maire-Mauritania: Targuist (Rif centr.).

C. laurifolius L.-Castella: Guadalajara.

C. Libanotis L. (C. Clusii Dun.).-Baetica: Venta del Pino (Almería).

C. monspeliensis L.-Barcelona.

C. salviifolius L.-Barcelona.

C. Sennenianus Pau-Mauritania: Dj. Ker-ker (Rif. or.), 1. clas.

Fumana ericoides (Cav.) Gandg. var. glandulosa Pau - Catalaunia:

Almatret. 
Halimium atriplicifolium Spach var macrocalycinum F. Q. et Maire -Mauritania: Ankod (Gomara), 1. clas.

\section{Violaceae}

Viola catalonica W. Becker-Barcelona: El Pujolet, 1. clas.

\section{Lythraceae}

Lythrum Salicaria L-Catalaunia: ad ripas fl. Gaià.

\section{Myrtaceae}

Myrtus communis L. fma. leucucarpa (DC).-Catalaunia: Blanes.

\section{Oenotheraceae}

Oenothera biennis L.-Catalaunia: Bellver (Cerdanya)

O. stricta Led.-Baetica: La Línea.

\section{Umbelliferae}

Amni Visnaga (L.) Lamk. var. Hybernonis (Sennen) Maire-Mauritania: Tauima (Rif or.)

Anethum graveolens L.-Leyde, ex horto botanico.

Angelica Archangelica L.-Ex herbariorum barcinonensium commercio. Bupleurum fruticosum L.-Barcelona.

B. montanum Coss.-Mauritania: Dj. Lexhab, c. Bab Taza.

B. protractum Hoff, et Link-Catalaunia: Platja d'Aro.

Capnophyllum peregrinum (L.) Lge.-Mauritania: R' gaia, c. Tandja.

Conium maculatum L.-Catalaunia: Manresa.

Coriandrum sativum L.-Frankfurt, ex horto botanico.

Eryngium Grosii F. Q.-Baetica: Cómpeta (1).

(1) Eryngium Grosii F. Q., sp. nova. (Seetio Campestria Wolff, subsectio Palmatisecta ej.).

Planta 10-30 cm alta, rhizomate longo, nigro, caulibus simplicibus apice plerumque trifurcatis vel ramis lateralibus plus minusve elongatis auctis edente. Foliis pallide glaucis, eximie coriaceis, vaide nervosis, inferioribus longe abrupteque petiolatis (petiolo ca. $6 \mathrm{~cm}$ lamina $4,5-5 \mathrm{~cm}$ longa), limbo ambitu reniforme (aurieulis exclusis $4,5-5 \times 7-9 \mathrm{~cm})$, a basi profunde cordato, palmato, trisecto, sectionibus pinnatis, tri-s. quinquepartitis, partitionibus obtegentibus, lobulatis, lobulis late triangularibus, spinosis; caulinis sessilibus s. subsessilibus, palmatisectis, minus divisis, segmentis angustioribus elongatisque. Bracteis involucri plerumque 6, ànguste lanceolatis, carinatis, supra medium bilobulato spinosis, a basi in utroque latere spinis $1-3,2-10 \mathrm{~mm} 1$. Capitulis hemisphaericis vel ovoideis, multifloris, 10-15 $\mathrm{mm}$ diam, bracteis floralibus integerrimis, linearibus, a basi dilatatis, subulato spinosis, floribus longioribus. Sepalis cordato lanceolatis in spinam eis dimidio breviorem abeuntis; petalis ca. - $\mathrm{mm}$ calyce brevioribus. Fructibus ca. $5 \mathrm{~mm}$ subtetragonis, dense squamatis, squamis alteris minutis alteris longis, praecipie superioribus longissimis, usque ad $1,5-2 \mathrm{~mm} \mathrm{1.,} \mathrm{lineari} \mathrm{lanceolatis,} \mathrm{a} \mathrm{basi} \mathrm{dilatatis,} \mathrm{apice}$ acutis. Planta tota albicans.

Hab. in Hispania meridionale, pr. Cómpeta (Málaga), 1. "Barranco de Juan Rojo", ubi cel. Gros, cui dicata species, d. 28 junii 1926 legit. 
Ferula communis L.-Baetica: Sierra Nevada. Et Pithyusae: Eivissa.

F. tingitana L. (F. Bolivari Pau, 1. clas.)-Mauritania: Dj. Gorgues, supra Tetauen.

Foeniculum officinale All.-Catalaunia: La Força (Montseny).

Heracleum Panaces L.-Dresden, ex horto botanico.

Heterothaenia thalictrifolia Boiss.-Baetica: Benaócaz (Cádiz).

Imperatoria hispanica Boiss.-Catalaunia: Santa Eulàlia de Ronçana.

Lagoecia cuminoides L.-Dresden, ex horto botanico.

Ligusticum lucidum Mill.-Catalaunia: Prades.

L. lucidum Mill, var. angustifolium Cuatrecasas-Baetica: Sierra Mágina (Jaén).

Magydaris panacifolia Lge.-Mauritania: El Araix.

Oenanthe callosa Salzm.-Mauritania: Dj. Zem-zem, inter Ceuta et Tetauen.

O. fistulosa L.-Catalaunia: Vilacolum.

Pastinaca lucida Gouan-Baleares: Aumallutx (Mallorea).

Scandix Balansae Reut.-Heidelberg, ex horto botanico.

Seseli Vayredanum F Q.-Baetica: El Chorro (Málaga).

Smyrnium perfoliatum L.-Baetica: Güéjar (Granada).

Thapsia decussata Lag.-Pithyusae: Eivissa.

T. villosa L.-Valentia: Banyeres.

Tinguarra sicula (L.) Benth. et Hook.-Mauritania: Tetauen.

\section{Cornaceae}

Cornus sanguinea L.-Barcelona.

\section{Primulaceae}

Cyclamen balearicum Willk.-Baleares: Mallorca.

Lysimachia Ephemerum L.-Catalaunia: Vila-vella.

L. minoricensis Rodr.-Baleares: Menorca.

\section{Plumbaginaceae}

Limonium Bonduelli (Letisb.) O. Kze.-Heidelberg, ex horto botanico. L. ornatum (Ball) O. Kze.-Mauritania: Haus.

Statice alliacea Cav.-Valentia: Serra Mariola.

S. Welwitschii (Boiss.) Samp. var. stenophylla (Welw.).-Coimbra, ex horto botanico.

\section{Apocynaceae}

Nerium Oleander L.-Barcelona, ubi colitur. 


\section{Asclepiadaceae}

Gomphocarpus fruticosus (L.) R. Br.-Barcelona.

Vincetoxicum nigrum Moench-Catalaunia: Balaguer.

\section{Borraginaceae}

Anchusa sempervirens L.-Castella: Hoyos del Espino (Avila).

\section{Verbenaceae}

Vitex Agnus-castus L.-Barcelona.

V. Negundo L. var. incisa Clarke-New-York, ex horto botanico.

\section{Labiatae}

Dracocephalum Mairei Emb.-Mauritania: Atlante medio.

Hyssopus officinaiis L.-Catalaunia: Biosca.

Lallemantia peltata F. et M.-Kobenhavn, ex horto botanico.

Lavandula dentata L. var. candicans Batt.-Mauritania: Axdir (Rif centr.) et Ifni (fma. inflorescentia magis tometosa).

L. latifolia Vill.-Barcelona: Castelldefels.

L. Mairei Humbert-Mauritania: Anti-Atlas.

L. maroccana Murb.-Atlante majore.

L. Staechas L.-Barcelona.

Leonurus Cardiaca L.-Frankfurt, ex horto botanico.

Nepeta Apuleji Ucr.-Baetica: Paterna.

N. Nepetella (L.) Koch var. leucophylla Lge--Valentia: Alacant, 1. clas.

Origanum elongatum Emb. et Maire,-Mauritania: Tizi Ifri (Atlante rifeo).

O. virens Hoffm. et Link-Coimbra, ex horto botanico.

Phlomis Caballeroi Pau-Mauritania: Punta Pescadores (Rif. occid.).

P. Lychnitis L.-Valentia: Serra Mariola.

P. maroccana Maire-Mauritania: Atlante medio.

P. tuberosa L.-Frankfurt, ex horto botanico.

Pitardia nepetoides Batt.-Mauritania: Taforalt (Beni Snassen).

Prasium majus L.-Madrid, ex horto botanico.

Salvia aegyptiaca L.-Mauritania: Beni Tuzin.

S. algeriensis Desf.-Mauritania: Dar Kebdani (Rif or.).

S. Barrelieri Etl,-Baetica: Alcalá de los Gazules.

S. Barrelieri Etl. var. pluripartita (Pau) Maire-Mauritania: EI Araix.

S. interrupta Schousb. ssp. Paui Maire-Mauritania: Xauen.

S. officinalis L.-Valentia: Serra Mariola.

S. officinalis L. var. vellerea Cuatr.-Baetica: Sierra Mágina.

S. patula Desf.-Mauritania: Zaian. 
S. phlomoides Asso fma. floribus caeruleis-Mauritania: Timhadit (Atlante medio).

S. Sclarea L.-Frankfurt, ex horto botanico.

S. Sclarea L. var. hispanica Gavioli-Catalaunia: Gualba.

S. tiliifolia Vahl-Kobenhavn, ex horto botanico.

Satureja candidissima (Munby) Briquet-Alger, ex horto botanico.

S. Hochreutineri Briquet-Mauritania: Atlante majore.

Sideritis Briquetiana F. Q. et Pau-Mauritania: Rif or. e plur. loc.

S. gossypina F. Q.-Mauritania: Ifni.

S. grandiflora Salzm.-Mauritania: Tetauen.

S. hyssopifolia L.-Heidelberg, ex horto botanico.

S. Lacaitae F. Q.-Baetica: Despeñaperros (Sierra Morena), 1, clas.

Stachys Fontqueri Pau-Mauritania: Tetauen, 1. clas.

S. Ocymastrum (L.) Briquet-Barcelona.

Teucrium Huotii Emb. et Maire var. Grosii F. Q.-Mauritania: Axdir (Rif centr.).

T. lancifolium Boiss.-Baleares: Galatzó (Mallorca).

T. Scorodonia L.-Catalaunia: Fanals d'Aro.

T. subspinosum Pourr. var. balearicum (Coss.) Pau-Baleares: Mallorca.

\section{Solanaceae}

Atropa baetica Willk.-Baetica: Sierra de la Nieve.

A. Belladona L.-Catalaunia: Serra de l'Almuçara.

Datura Metel L.-Catalaunia: Calafell.

D. Stramonium L.-Catalaunia: La Força (Montseny).

Hyoscyamus niger L.-Catalaunia: Sant Marçal (Montseny).

Physalis Alkekengi L.-Catalaunia: Sant Guim.

Solanum Dulcamara L.-Catalaunia: Prades.

S. Lycopersicum L. proles vel var. ex Ifni-Elata (in horto botanico barcinonense usque ad $4 \mathrm{~m}$ alt.) infrutescentia constanter quinquebaccata fructibus sphaericis, $15-18 \mathrm{~mm}$ diam. Affinis videtur Solano racemifloro Dun. Colitur in hortis macerrimis ifniensibus.

S. Sodomaeum L.-Baetica: San Fernando.

Triguera Osbeckii (L.) Willk.-Baetica: Medina Sidonia.

Withania somnifera L.-Mauritania: Saf-saf (Rif. or.).

\section{Scrofulariaceae}

Antirrhinum chrysothales F. Q.--Mauritania: Axdir (Rif centr.).

A. Lazari Sennen (gr. majus),-Mauritania: Melilla.

A. ramosissimum Coss. et Dur.-Mauritania: Ifni.

A. tortuosum Bose var. osmarense Pau-Mauritania: Tetauen, 1. cl.

Digitalis lutea L.-Catalaunia: La Molina. 
D. obscura L.-Catalaunia: Tarragona.

D. purpurea L. var. mauritanica Humb. et Maire-Mauritania: Dj. Iguermalez (Atlante rifeo).

D. Thapsi L.-Extremadura: Mirabel.

Linaria melanantha Boiss. et Reut.-Legion: Cabrera Baja.

L. ventricosa Coss.-Mauritania: Sus.

Scrofularia canina L.-Mauritania: Ifni.

S. eriocalyx Emb. et Maire-Mauritania: Zarkat (Atlante rifeo).

S. mellifera Vahl-Baetica: Algeciras.

S. peregrina L.-Ruscino: Cotlliure.

S. sambucifolia L.-Mauritania: Bab Taza.

Verbascum Blattaria L.-Catalaunia: La Cellera.

\section{Martyniaceae}

Martynia fragrans Lindl.-Dresden, ex horto botanico.

\section{Globulariaceae}

Globularia Alypum L.-Barcelona: Castelldefeìs.

\section{Acanthaceae}

Acanthus mollis L.-Barcelona, colitur.

\section{Plantaginaceae}

Plantago albicans L. (P. albicantiformis Senn.!)-Mauritania: Muluia (Rif or.).

P. mauritanica Boiss. et Reut.-Mauritania: Beni Mhamed (Beni Zedjel).

P. ovata Forsk.-Baetica: Cuevas (Almeria).

\section{Rubiaceae}

Galium pruinosum Boiss.-Baetica: Málaga.

\section{Valerianaceae}

Fedia Cornucopiae Gaertn.-Mauritania: Beni Arós.

Kentranthus macrosiphon Boiss.-Dresden, ex horto botanico.

\section{Caprifoliaceae}

Leycesteria formosa Wall.-New-York, ex horto botanico.

Lcnicera implexa Ait.—Barcelona.

Sambucus nigra L-Catalaunia: Osor.

Viburnum Tinus L.-Barcelona. 


\section{Dipsacaceae}

Callistemma brachiatum (Sibth.) Boiss.-Kobenhavn, ex horto botanico. Cephalaria Fragosoana Pau - Mauritania: Xauen.

C. mauritanica Pomel ssp. rifana Maire-Mauritania: Dj. Lexhab, c. Bab Taza.

\section{Cucurbitaceae}

Bryonia dioica L.-Catalaunia: Prades.

Ecballium Elaterium Rich.-Barcelona.

\section{Campanulaceae}

Campanula affinis Roem. et Sch.-Catalaunia: Montserrat, 1. clas. C. afra Cav.-Baetica: Estepona.

Platycodon grandiflorum (Jacq.) A. DC.-Leyde, ex horto botanico. Trachelium caeruleum L.-Alger, ex horto botanico.

\section{Compositae}

Anacyclus radiatus Lois.-Marseille, ex horto botanico.

A. radiatus Lois, fma. sulphu reus Br.-Bl. et Maire-Marseille, ex h. b.

A. radiatus Lois. fma. discoideus Chiovenda.-Marseille, ex h. b. Andryala mogadorensis Coss. et Bal.-Mauritania: Mogador, 1. clas. Anthemis maritima L.-Baetica: Cádiz.

A. mixta L.-Catalaunia: Platja d'Aro.

A. tinctoria L.-Leningrad, ex horto botanico.

Arctium Lappa L.-Barcelona.

A. tomentosum (Lamk.) Schrank-Dresden, ex horto botanico.

Calendula marginata Willd.-Baetica: Gibraltar, 1. clas.

C. suffruticosa Vahl -Alger, ex horto botanico.

C. suffruticosa Vahl var. aestivalis Sennen-Mauritania: Kadia (Rif. or.).

Carduncellus pinnatus (Desf.) DC. var. acaulis Presl -Mauritania: Beni Derkul, 1. Hauta-el-Kasdir.

Carduus Gayanus Dur.-Asturia: Cangas de Tineo.

C. granatensis Willk. var, tetuanensis Pau-Mauritania: Tetauen, 1. clas. Carthamus arborescens L.-Mauritania: Beni Selman.

$\times$ C. Martis F. Q. --C. arborescens $q \times$ C. ripheus $\delta$ ej.-Semina fertilia generationis $\mathrm{I}$ ex $\mathrm{H}$. B. Barcinonense possibiliter vicinitate ratione polline parentum fecundata.

C. riphaeus F. Q.-Mauritania: Snada (Rif occid.).

Centaurea Clementei Boiss.-Baetica: Ronda.

C. crocodylium L.-Kobenhavn, ex horto botanico.

C. diluta Ait.-Baetica: Paterna.

C. dracunculifolia Duf.-Barcelona: Prat de Llobregat.

C. fragilis Dur. var. subinermis Pau-Mauritania: Melilla. 
C. involucrata Desf.-Mauritania: Melilla.

C. leucantha Pourr. var. grandifolia F. Q.-Pithyusae: Formentera.

C. pullata L.-Mauritania: Tetauen.

C. sempervirens L.-Coimbra, ex horto botanico.

C. sempervirens L. var. algerica Maire-Alger, ex horto botanico.

C. sempervirens L. ssp. mauritanica F. Q.-Mauritania: Xauen, 1. clas. Species gigantea, in $\mathrm{H}$. B. Barcinonense usque ad $4 \mathrm{~m}$ arbusculis fulta.

$\times$ C. Caroli-Paui F. Q. $=$ C. sempervirens var. algerica $q \times$ C. sempervirens ssp. mauritanica $\delta$ ej. Semina fertilia generationis I e planta autofecundata.

C. uliginosa Brot.-Coimbra, ex horto botanico.

Cinara humilis L.-Baetica: Ubrique.

C. Hystrix Ball.-Mauritania: c. Targuist (Rif, centr).

Cirsium (Chamaepeuce) leptophyllum F. Q.-Mauritania: Dj. Iguermalez, 1. clas.

C. (Chamaepeuce) rhiphaeum F. Q.-Mauritania: Ktama, 1, clas.

C. riphaeum F. Q. var, tetauense F. Q.-Mauritania: Dj. Gorgues, supra Tetauen, 1. clas.

C. riphaeum F. Q. ssp. abylense F. Q.-Mauritania: Monte Abyla, c. Ceuta, l. clas.

C. scabrum (Poir.) Bonnet et Barratte-Mauritania: El Araix.

C. tuberosum All.-Catalaunia: Albarca.

Cladanthus arabicus Cass.-Paris, ex horto botanico.

Cnicus benedictus L.-Catalaunia: Balaguer.

Crepis Triasii Willk.-Baleares: Mallorca.

Echinops Font-Queri Pau.-Mauritania: Xauen, 1. clas.

E. spinosus L.-Mauritania: Axdir (Rif centr.).

Galinsoga parviflora Cav.-Riga, ex horto botanico.

Glossopappus macrotus (Dur.) Briq.-Baetica: Utrera.

Grindelia robusta Nutt.-Kobenhavn, ex horto botanico.

Helminthia echioides Gaertn.-Barcelona.

Hertia maroccana Maire - Mauritania: Tafilalet.

Hieracium virosum Pall.-Leningrad, ex horto botanico.

Hypochaeris (Seriola) laevigata (L.) C., P. et G.-Mauritania: Xauen. Inula Helenium L.-Warszawa, ex horto botanico.

Lactuca cornigera F. Q. et Pau -Mauritania: Ktama, 1. clas.

Launaea arborescens (Batt) Maire - Mauritania: Beni Said.

L. cervicornis (Boiss.) F. Q. et Rothm.-Baleares: Mallorca.

Leontodon maroccanus (Pers.) Ball-Mauritania: EI Araix.

Leucanthemum demnatense Murb.-Mauritania: Ktama.

L. paludosum (Poir.) Bonnet-Mauritania: Amekran, c. Axdir (Rif centr.).

L. osmarense (Ball) F. Q. mutatio macrocephalum - In Horto Botanico 
Barcinonense e seminibus montis Beni Osmar (Mauritania) enata. Lindheimeria texana A. Gray-Dresden, ex horto botanico. Micropus supinus L.-Mauritania: Bu-Halla (Beni Derkul). Microseris linearifolia (DC.) A. Gray -Penza, ex horto botanico. Oņopordon dissectum Murb. ssp. Murbeckii Lindb. var. lixense MaireMauritania: El Araix, 1. clas.

$\times$ O. plutonii F. Q., hybr. nov.-O dissectum ssp. Murbeckii var. lixense $\times 0$. illyricum ej. Seminibus generationis II formarum a parentibus exacte intermedia et $\beta$ (O. illyricum $>$ Murbeckii) et $\gamma(0$. illyricum $<$ Murbeckii) omnibus autofecundatis (1).

o. illyricum L.-Barcelona.

O. nervosum Boiss. var. glomeratum Costa - Catalaunia: Margalef. Ormenis scariosa (Ball) Lit. et Maire-Alger, ex horto botanico.

Palafoxia texana DC.-Dresden, ex horto botanico.

Perralderia Paui F. Q.-Mauritania: Dj. Asru (Rif or.). Phagnalon calycinum (Cav) DC.-Mauritania: Mogador, 1. clas. Podospermum laciniatum DC.-Barcelona. Rhaponticum acaule (L.) DC.-Mauritania: Dj. Arruit (Rif. or.). Santolina pectinata Lag.-Mauritania: Dj. Kalaa, supra Xauen. Scorzonera pygmaea Sibth. et Sm.-Mauritania: Targuist (Rif. centr.). Sylibum eburneum Coss. et Dur. var. hispanicum Willk.-Castella: Aranjuez.

Sonchus aquatilis Pourr.-Catalaunia: Amposta.

S. Mas-Guindalii Pau et F. Q.-Mauritania. Bocoia.

Tagetes glandulifera Schk.-Barcelona: Castelldefels, introducta.

Volutaria crupinoides (Desf.) Maire - Mauritania: El Garman (Rif. or.). V. muricata (L.) Maire-Mauritania.

Xeranthemum inapertum (L.) Willd. var. Reboudianum Verlot-Mauritania: Atlante majore.

Barcinone, 31 decembris 1938.

\section{Dr. P. Font Quer}

Horti Director

\author{
J. Santamaria \\ Horti Magister
}

\author{
M. Clarassó \\ Horti Magister
}

Adresse: Institut Botànic, carrer de S. Gervasi, 94. - BARCELONA.

(1) Onopordon Plutonii F. Q., hybr. nov. =0. dissectum ssp. Murbeckii var.

Ab $O$. Murbeckii differt

foliorum segmentis minus profule robustiore ad apicem usque longe alato spinoso, longioribus latioribusque, inferioribus refibus, spinisque validis, anthodii squamis illyrico caule elatiore, ramis erioribus reflexis in spinam tenuem abeuntis. Ab 0 . lis munitis ideoque longe nudis, foliorum gracilioribus, apicem versus foliis parvuanthodi squamis magis angustis et numerosis.

In Horto Botanico Barcinonense, inter parentes. 
